Publ. RIMS, Kyoto Univ.

35 (1999), 515-570

\title{
Blowing Ups of 3-dimensional Terminal Singularities
}

By

Takayuki HAYAKAwA *

\begin{abstract}
We study the blowing up $\pi: \bar{X} \rightarrow X$ of a 3-dimensional terminal singularity $X$ of index $m \geq 2$ such that the exceptional locus of $\pi$ consists of a prime divisor $E$ with discrepancy $1 / \mathrm{m}$. A complete classification of such blowing ups is given and it is proved that these correspond to weighted blow ups by a certain kind of maximal weights except for the case where $X$ is of type $(\mathrm{cD} / 2)$. We shall treat the $(\mathrm{cD} / 2)$ case later. These also give examples of contractions of extremal rays which contract a divisor to a point.
\end{abstract}

\section{Contents}

$\S 1$. Introduction

$\S 2$. Classification of 3-dimensional Terminal Singularties

$\S 3$. Weighted Blow Ups and Weighted Valuations

$\S 4$. Main Results and Comments on the Proof

$\S 5$. Some Auxiliary Results

\& 6 . Terminal Singularities of Type $(\mathrm{cA} / \mathrm{m})$

\& 7. Terminal Singularities of Type (cAx/4)

\& 8. Terminal Singularities of Type $(\mathrm{cAx} / 2)$

\& 9. Terminal Singularities of Type $(\mathrm{cD} / 3)$

$\S 10$. Terminal Singularities of Type (cE/2)

References

Communicated by S. Mori, October 14, 1998.

1991 Mathematics Subject Classification : 14B05 and 32B30.

* Department of Mathematics, Faculty of Science, Kanazawa University, Kakuma-machi, Kanazawa 920-1192, Japan. 


\section{$\S \mathbb{1}$. Introduction}

Let $\bar{X}$ be a projective 3 -fold with only terminal singularities defined over the complex number field $\mathbb{C}$. If the canonical divisor $K_{\bar{X}}$ of $\bar{X}$ is not nef, then there is a projective surjective morphism $\pi: \bar{X} \rightarrow X$, which is the contraction of an extremal ray. If $\operatorname{dim} X<3$, then $\pi$ is called a fiber type contraction. If $\operatorname{dim} X=3$, then $\pi$ is a birational morphism. Let $E$ be the exceptional locus of $\pi$. Then $\pi$ is called a divisorial contraition if $E$ is an irreducible divisor and called a flipping contraction if $\operatorname{dim} E=1$.

The most difficult part of the Minimal Model Program was to construct the flip $\pi^{+}: \bar{X}^{+} \rightarrow X$ when $\pi: \bar{X} \rightarrow X$ is a flipping contraction. This was established by [Mori88] and the structure of flipping contractions and their flips are wellunderstood by [KM92].

Divisorial contractions $\pi: \bar{X} \rightarrow X$ were considered to be the easy part of the Minimal Model Program since $X$ has only terminal singularities. However the detailed description of these are not known except for a few cases :

(1) If $\bar{X}$ is smooth or has only Gorenstein terminal singularities, then there is a complete list of $\pi$ ([Mori82], [Cut88]).

(2) If $X$ has a cyclic quotient terminal singularity, then $\bar{X}$ is obtained by a weighted blow up ([Kaw96]).

(3) If the index of $\bar{X}$ is not greater then that of $X$, then $X$ is restricted to a very few cases and we can construct $\pi$ explicitly ([Luo98]).

Recently it seems that a classification of divisorial contractions is indispensable for the birational study of 3-folds ([Cor95], [Ko197]).

In this paper, we shall study divisorial contractions $\pi: \bar{X} \rightarrow X$ which contract an irreducible divisor to a point under some assumptions. Since $X$ has only terminal singularities, we start with a germ of a 3-dimensional terminal singularity $X$ and look for projective birational morphisms $\pi: \bar{X} \rightarrow X$ which give divisorial contractions. Examples in [Kaw96] and some explicit calculations indicate that the discrepancy of the exceptional divisor $E$ of $\pi$ is small in many cases. We shall adopt this as our assumption. Thus our problem becomes as follows :

For each germ of a 3-dimensional terminal singularity $X$ of index $m$, find all projective morphisms $\pi: \bar{X} \rightarrow X$ such that

(i) $\bar{X}$ has only terminal singualrities,

(ii) the exceptional divisor $E$ of $\pi$ is irreducible, and

(iii) $K_{\bar{X}}=\pi^{*}\left(K_{X}\right)+\frac{1}{m} E$.

Such a morphism $\pi$ will be called a divisorial blow up with discrepancy $1 / \mathrm{m}$.

The purpose of this paper is to determine all divisorial blow ups of $X$ with discrepancies $1 / m$ if $X$ is a germ of a 3-dimensional terminal singularity of index $m$ $\geq 2$. Our main results says that this is possible except for the $(c D / 2)$ case. These are all obtained by weighted blow ups and there is a one-to-one correspondence between these blow ups and certain set of weights. Moreover we found that the 
axial weight will not increase after a divisorial blow up with discrepancy $1 / m$. By studying singularities more carefully, we know that we can resolve the singularitiy of $X$ by a succesion of these blow ups. These are summarized in Section 4 in a precise form. For the $(\mathrm{cD} / 2)$ case, we can also determine all divisorial blow ups with discrepancies $1 / 2$, but some of them are obtained by a different type of weighted blow ups and the one-to-one correspondence as above does not necessarily hold. So we shall not include these results here and we treat the (cD/2) case separately.

By [Kaw93], there is at least one divisor which has discrepance $1 / m$ over $X$ (this also holds if $X$ is of index 1 by [Mar96]). Our method to find divisorial blow ups with discrepancies $1 / m$ is similar to the one in [Kaw93] and sometimes we use the same weighted blow ups. In [Kaw93], the blown up varieties may have nonterminal singularities or the exceptional divisors may not be irreducible. By studying these blow ups more carefully, we can determine all the divisorial blow ups with discrepancy $1 / m$ completely.

This paper is organized as follows : In Section 2, we recall the results on classification of 3-dimensional terminal singularities and some definitions. In Section 3, we review the notion of weighted blow ups and discrepancies of divisors. The notation and definitions in these sections are used later. Main results and some of their corollaries are summarized in Section 4 with some comments on the proofs. In Section 5, we shall show the results on cyclic quotient terminal singularities and some lemmas which is used to estimate the number of divisors with discrepancies $1 / m$. Sections $6-10$ are devoted to proving our main results. We shall use the classification of 3-dimensional terminal singularities.

The author would like to thank Professor S. Mori for his invaluable suggestions and encouragement.

Notation. The following are the notation which we shall use frequently in this paper.

(1) For a rational number $x$, we denote its integral part by $[x]$ and its fractional part by $\langle x\rangle$, i. e. $[x]$ satisfies $[x] \in \mathbb{Z}, x-1<[x] \leq x$, and $\langle x\rangle=x-[x]$.

(2) Let $f \in \mathbb{C}\left\{x_{1}, \ldots, x_{n}\right\}$ and let $M$ be a monomial. We write $M \in f$ if the coefficient of $M$ in the power series expansion of $f$ is nonzero.

(3) For $f(x)=\sum a_{n} x^{n} \in \mathbb{C}\{x\}$, we define ord $(f(x))=\min \left\{n \mid a_{n} \neq 0\right\}$.

\section{§ 2. Classification of 3-dimensional Terminal Singularities}

In this section we fix our notation and summarize the results on classification of 3-dimensional terminal singularities, which will be used in the following sections.

2.1. We denote the complex space $\mathbb{C}^{n}$ with coordinates $x_{1}, \ldots, x_{n}$ by $\left(x_{1}, \ldots\right.$, 
$\left.x_{n}\right)$. Let $\mathbb{Z}_{m}$ be a cyclic group of order $m$. We define the action of $\mathbb{Z}_{m}$ on $\left(x_{1}, \ldots\right.$, $\left.x_{n}\right)$ by $\tau\left(x_{1}\right)=\zeta^{\alpha}{ }_{1} x_{1}, \ldots, \tau\left(x_{n}\right)=\zeta^{\alpha}{ }_{n} x_{n}$ where $\tau$ is a generator of $\mathbb{Z}_{m}, \zeta$ is a primitive root of unity and $\alpha_{1}, \ldots, \alpha_{n}$ are integers. The quotient space of $\left(x_{1}, \ldots, x_{n}\right)$ is denoted by $\left(x_{1}, \ldots, x_{n}\right) / \mathbb{Z}_{m}\left(\alpha_{1}, \ldots, \alpha_{n}\right)$ or $\left(x_{1}, \ldots, x_{n}\right) / \mathbb{Z}_{m}$ or $\mathbb{C}^{n} / \mathbb{Z}_{m}$ if there would be no confusion.

Let $\varphi\left(x_{1}, \ldots, x_{n}\right) \in \mathbb{C}\left\{x_{1}, \ldots, x_{n}\right\}$ be a $\mathbb{Z}_{m}$-semi-invariant. Then $\mathbb{Z}_{m}$ also acts on the germ of the hypersurface $\left\{\varphi\left(x_{1}, \ldots, x_{n}\right)=0\right\} \subseteq\left(x_{1}, \ldots, x_{n}\right)$. We denote the quotient space by $\left\{\varphi\left(x_{1}, \ldots, x_{n}\right)=0\right\} / \mathbb{Z}_{m}\left(\alpha_{1}, \ldots, \alpha_{n}\right)$ or $\left\{\varphi\left(x_{1}, \ldots, x_{n}\right)=0\right\} / \mathbb{Z}_{m}$, which we call a hyperquotient singularity.

In this paper, we mainly deal with the $n=4$ case and we shall often use the coordinates $x, y, z, u$ (in this order) instead of $x_{1}, x_{2}, x_{3}, x_{4}$. So $\mathbb{C}^{4}$ with these coordinates will be denoted by $(x, y, z, u)$.

Now we state the results on classification of 3-dimensional terminal singularities. The first one is due to [Reid83] which deals with terminal singularities of index 1, and the second one is due to [Dan83], [MS84] and [Mori85] which treats the case where index $\geq 2$.

2.2. Theorem. A 3-dimensional singularity is terminal of index 1 if and only if it is an isolated $c D V$ point.

2.3. Theorem. Let $X$ be a germ of a 3-dimensional terminal singularity of index $\geq 2$. Then there is an embedding $j: X \hookrightarrow(x, y, z, u) / \mathbb{Z}_{m}$ such that one of the following holds :

$(\mathrm{cA} / \mathrm{m}) X \simeq\{x y+f(z, u)=0\} / \mathbb{Z}_{m}(\alpha,-\alpha, 1,0)$ where $\alpha$ is an integer prime to $m$ and $f(z, u) \in \mathbb{C}\{z, u\}$ is a $\mathbb{Z}_{m}$-invariant.

$(\mathrm{cAx} / 4) \quad X \simeq\left\{x^{2}+y^{2}+f(z, u)=0\right\} / \mathbb{Z}_{4}(1,3,1,2)$ where $f(z, u) \in \mathbb{C}\{z, u\}$ is a $\mathbb{Z}_{4}$ semi-invariant and $u \notin f(z, u)$.

$(\mathrm{cAx} / 2) X \simeq\left\{x^{2}+y^{2}+f(z, u)=0\right\} / \mathbb{Z}_{2}(0,1,1,1)$ where $f(z, u) \in(z, u)^{4} \mathbb{C}\{z, u\}$ is a $\mathbb{Z}_{2}$-invariant.

$(\mathrm{cD} / 3) X \simeq\{\varphi(x, y, z, u)=0\} / \mathbb{Z}_{3}(1,2,2,0)$ where $\varphi$ has one of the following forms :

(cD/3-1) $\varphi=u^{2}+x^{3}+y z(y+z)$,

(cD/3-2) $\varphi=u^{2}+x^{3}+y z^{2}+x y^{4} \lambda\left(y^{3}\right)+y^{6} \mu\left(y^{3}\right)$ where $\lambda\left(y^{3}\right), \mu\left(y^{3}\right) \in \mathbb{C}\left\{y^{3}\right\}$ and $4 \lambda^{3}+27 \mu^{2} \neq 0$,

(cD/3-3) $\varphi=u^{2}+x^{3}+y^{3}+x y z^{3} \alpha\left(z^{3}\right)+x z^{4} \beta\left(z^{3}\right)+y z^{5} \gamma\left(z^{3}\right)+z^{6} \delta\left(z^{3}\right)$ where $\alpha\left(z^{3}\right), \beta\left(z^{3}\right), \gamma\left(z^{3}\right), \delta\left(z^{3}\right) \in \mathbb{C}\left\{z^{3}\right\}$.

(cD/2) $X \simeq\{\varphi(x, y, z, u)=0\} / \mathbb{Z}_{2}(1,1,0,1)$ where $\varphi$ has one of the following forms :

(cD/2-1) $\varphi=u^{2}+x y z+x^{2 a}+y^{2 b}+z^{c}$ where $a, b \geq 2, c \geq 3$,

(cD/2-2) $\varphi=u^{2}+y^{2} z+\lambda y x^{2 a+1}+g(x, z)$ where $\lambda \in \mathbb{C}, a \geq 1, g(x, z) \in\left(x^{4}, x^{2} z^{2}\right.$, $\left.z^{3}\right) \mathbb{C}\{x, z\}$. 
$(\mathrm{cE} / 2) X \simeq\left\{u^{2}+x^{3}+g(y, z) x+h(y, z)=0\right\} / \mathbb{Z}_{2}(0,1,1,1)$ where $g(y, z) \in$ $(y, z)^{4} \mathbb{C}\{y, z\}, h(y, z) \in(y, z)^{4} \mathbb{C}\{y, z\} \backslash(y, z)^{5} \mathbb{C}\{y, z\}$.

The index of $X$ is equal to the order of the cyclic group $\mathbb{Z}_{m}$.

2.4. For each 3-dimensional terminal singularity $X$ of index $m \geq 2$, there is an embedding $j: X \hookrightarrow(x, y, z, u) / \mathbb{Z}_{m}$ as in (2.3). We fix one of such embedding and call it a standard embedding of $X$.

The following result is due to [KSB88] (see also [Ste88]), which completes the classification of 3-dimensional terminal singularities.

2.5. Theorem. Let $X$ be one of the hyperquotient singularity $\{\varphi(x, y, z, u)=$ $0\} / \mathbb{Z}_{m}$ listed in (2.3). Assume that $\varphi(x, y, z, u)=0$ defines an isolated singularity at (0) and that the action of $\mathbb{Z}_{m}$ is free outside (0). Then $X$ is terminal.

2.6. Axial weights. Let $X=\{\varphi(x, y, z, u)=0\} / \mathbb{Z}_{m}(\alpha, \beta, \gamma, \delta)$ be a germ of a 3-dimensional terminal singularity of index $m \geq 2$ at the origin $P \in X$ as in (2.3). Then there is a linear form $l \in \mathbb{C}\{x, y, z, u\}$ such that $\varphi+l$ is a $\mathbb{Z}_{m}$-semi-invariant. For a $\mathbb{Z}_{m}$-invariant open neighborhood $U$ of the origin of $(x, y, z, u)$, let

$$
\mathscr{X}=\{(x, y, z, u, t) \in U \times \mathbb{C} \mid \varphi(x, y, z, u)+t l(x, y, z, u)=0\} / \mathbb{Z}_{m}(\alpha, \beta, \gamma, \delta, 0),
$$

and let $p: \mathscr{X} \rightarrow \mathbb{C}$ be the projection to the $t$-axis. If $U$ is sufficiently small and if $0<|t| \ll 1$, then $p^{-1}(t)$ has only cyclic quotient terminal singularities (see [Kaw 86]). The number of cyclic quotient singularities of $p^{-1}(t)$ is called the axial weight of $X$ at $P$. We shall denote this by aw $(X, P)$ or simply aw $(X)$ if there would be no confusion. If $X$ is smooth or has an isolated $\mathrm{cDV}$ point at $P$, then we shall define aw $(X, P)=1$.

Using notation of (2.3), the explicit values of axial weights are as follows :

$$
\operatorname{aw}(X)= \begin{cases}\operatorname{ord}(f(0, u)) & \text { if } X \text { is of type }(\mathrm{cA} / \mathrm{m}), \\ (\operatorname{ord}(f(0, u)+1) / 2 & \text { if } X \text { is of type }(\mathrm{cAx} / 4), \\ 2 & \text { if } X \text { is of type }(\mathrm{cAx} / 2) \text { or }(\mathrm{cD} / 3), \\ c & \text { if } X \text { is of type }(\mathrm{cD} / 2-1), \\ \operatorname{ord}(g(0, z)) & \text { if } X \text { is of type }(\mathrm{cD} / 2-2), \\ 3 & \text { if } X \text { is of type }(\mathrm{cE} / 2) .\end{cases}
$$

This notion coincides with the notion of "weight" in [Morr86] and "axial multiplicity" in [Mori88] except for the (cAx/4) case. If $X$ is of type (cAx/4), 
then these three notions are all different.

2.7. Divisorial blow ups. Let $X$ be a germ of a 3-dimensional terminal singularity.

By a partial resolution, we mean a projective birational morphism $\phi: Z \rightarrow X$ such that $Z$ has only terminal singularities. For a $\mathbb{Q}$-Cartier Weil divisor $D$ on $X$, the proper transform of $D$ by $\psi$ will be denoted by $\psi^{-1}[D]$.

Let $\phi: Z \rightarrow X$ be a partial resolution of $X$ and let $\Sigma E_{i}$ be the exceptional divisor of $\phi$. Then we can write

$$
K_{Z}=\phi^{*}\left(K_{X}\right)+\sum a_{i} E_{i},
$$

where $0<a_{i} \in \mathbb{Q}$. The coefficient $a_{i}$ is called the discrepancy of $E_{i}$ over $X$ and it is denoted by $a\left(E_{i}, X\right)$. The discrepancy of $E_{i}$ only depends on the discrete valuation on the function field of $X$ associated to $E_{i}$ and does not depend on the choice of $\psi$. Thus we shall often identify prime divisors with the corresponding discrete valuations when we speak about "divisors over X".

A projective birational morphism $\pi: \bar{X} \rightarrow X$ is called a divisorial blow up with discrepancy $\alpha(>0)$ if the following conditions are satisfied :

(i) $\bar{X}$ has only terminal singularities,

(ii) the exceptional set of $\pi$ is an irreducible divisor $E$, and

(iii) $K_{\bar{X}}=\pi^{*}\left(K_{X}\right)+\alpha E$.

Moreover, if $\pi$ is a blow up, then we shall often say that $\pi$ is divisorial with discrepancy $\alpha$.

In this paper, we are interested in divisorial blow ups with discrepancies $1 / \mathrm{m}$ where $m$ is the index of $X$.

\section{§ 3. Weighted Blow Ups and Weighted Valuations}

3.1. Let $Y=\left(x_{1}, \ldots, x_{n}\right) / \mathbb{Z}_{m}\left(\alpha_{1}, \ldots, \alpha_{n}\right)$ be a cyclic quotient singularity. We can describe this by using the theory of toric varieties (cf. [Oda88], [Fu193]). Let

$$
e_{1}=(1,0, \ldots, 0), \ldots, e_{n}=(0, \ldots, 0,1) \text { and } e=\frac{1}{m}\left(\alpha_{1}, \ldots, \alpha_{n}\right) \text {. }
$$

Then $Y=\left(x_{1}, \ldots, x_{n}\right) / \mathbb{Z}_{m}\left(\alpha_{1}, \ldots, \alpha_{n}\right)$ is the toric variety corresponding to the lattice $N=\mathbb{Z} e_{1}+\cdots+\mathbb{Z}_{n}+\mathbb{Z} e$ and the cone $C=\mathbb{R}_{\geq 0} e_{1}+\cdots+\mathbb{R}_{\geq 0} e_{n}$, precisely $N$ and the fan $\Delta$ consisting of all the faces of $C$.

3.2. Weighted blow up. Let $\sigma=\frac{1}{m}\left(a_{1}, \ldots, a_{n}\right) \in N$ be an element with $a_{1}$, $\ldots, a_{n}>0$ and assume that $e_{1}, \ldots, e_{n}$ and $\sigma$ generate the lattice $N$. Such $\sigma \in N$ will be called a weight. We can construct the weighted blow up $\bar{\pi}: \bar{Y} \rightarrow Y=\left(x_{1}, \ldots, x_{n}\right) /$ 
$\mathbb{Z}_{m}$ with weight $\sigma$ as follows (cf. [KM92, 10]):

We divide the cone $C$ by adding the 1 -dimensional cone $\mathbb{R}_{\geq 0} \sigma$, that is, we divide $C$ into $n$ cones :

$$
C_{i}=\mathbb{R}_{\geq 0} e_{1}+\cdots+\mathbb{R}_{\geq 0}^{i \text { th }} \sigma+\cdots+\mathbb{R}_{\geq 0} e_{n} \quad(i=1, \ldots, n) .
$$

Let $\Delta^{\prime}$ be the fan consisting of all the faces of $C_{1}, \ldots, C_{n}$. Then $\bar{Y}$ is the toric variety corresponding to $N$ and $\Delta^{\prime}$, and $\bar{\pi}$ is the morphism which is induced from the natural map of fans $\left(N, \Delta^{\prime}\right) \longrightarrow(N, \Delta)$.

The variety $\bar{Y}$ is covered by $n$ affine open sets $\bar{U}_{1}, \ldots, \bar{U}_{n}$ which corresponds to the cones $C_{1}, \ldots, C_{n}$ respectively. These affine open sets and $\bar{\pi}$ are described as follows :

$$
\begin{gathered}
\bar{U}_{i}=\left(\bar{x}_{1}, \ldots, \bar{x}_{n}\right) / \mathbb{Z}_{a_{i}}\left(-a_{1}, \ldots, \stackrel{i \text { th }}{m}, \ldots,-a_{n}\right) \\
\left.\bar{\pi}\right|_{\bar{U}_{i}}: \bar{U}_{i} \ni\left(\bar{x}_{1}, \ldots, \bar{x}_{n}\right) \longmapsto\left(\bar{x}_{1} \bar{x}_{i}^{a_{l} / m}, \ldots, \bar{x}_{i}^{a^{t} \text { th }}{ }^{a_{m}}, \ldots, \bar{x}_{n} \bar{x}_{l}^{a_{i} / m}\right) \in Y .
\end{gathered}
$$

The exceptional divisor $\bar{E}$ of $\bar{\pi}$ is isomorphic to the weighted projective space $\mathbb{P}\left(a_{1}, \ldots, a_{n}\right)$.

3.3. Let $\varphi\left(x_{1}, \ldots, x_{n}\right) \in \mathbb{C}\left\{x_{1}, \ldots, x_{n}\right\}$ be a $\mathbb{Z}_{m}$-semi-invariant. For a hyperquotient singularity $X=\left\{\varphi\left(x_{1}, \ldots, x_{n}\right)=0\right\} / \mathbb{Z}_{m} \subseteq\left(x_{1}, \ldots, x_{n}\right) / \mathbb{Z}_{m}$, let $\bar{X}=\bar{\pi}^{-1}[X]$ be the proper transform of $X$ by $\bar{\pi}$ and let $\pi=\left.\bar{\pi}\right|_{\bar{X}}$ be the restriction of $\bar{\pi}$. Then $\pi: \bar{X} \longrightarrow X$ is also called the weighted blow up with weight $\sigma$ or simply the $\sigma$-blow up. Furthermore, we set $U_{i}=\left.\bar{U}_{i}\right|_{\bar{X}}$ for $i=1, \ldots, n$. Each $U_{i}$ is a hyperquotient singularity in $\bar{U}_{i}$ and $\bar{X}$ is covered by $U_{1}, \ldots, U_{n}$. In this paper, $U_{i}$ (resp. $\bar{U}_{i}$ ) is called the $x_{i}$-chart of $\bar{X}$ (resp. $\bar{Y}$ ).

3.4. Let $\sigma=\frac{1}{m}\left(a_{1}, \ldots, a_{n}\right) \in N$ be a weight. We define the function

$$
\sigma-w t: \mathbb{C}\left\{x_{1}, \ldots, x_{n}\right\} \longrightarrow \mathbb{Q}
$$

as follows :

First we put $\sigma$-wt $\left(x_{1}\right)=a_{1} / m, \ldots, \sigma-w t\left(x_{n}\right)=a_{n} / m$. (We shall often abbreviate this as $\sigma$-wts $\left(x_{1}, \ldots, x_{n}\right)=\frac{1}{m}\left(a_{1}, \ldots, a_{n}\right)$.) Next for monomials $M=x_{n}^{p_{1}} \ldots x_{n}^{p_{n}}$, we define

$$
\sigma-w t(M)=p_{1} \sigma-w t\left(x_{1}\right)+\cdots+p_{n} \sigma-w t\left(x_{n}\right)=\left(p_{1} a_{1}+\cdots p_{n} a_{n}\right) / m
$$

Finally for general $f=\sum_{I} \alpha_{I} M_{I}, \alpha_{I} \in \mathbb{C}, M_{I}$ : monomials, we define

$$
\sigma-w t(f)=\min \left\{\sigma-w t\left(M_{I}\right) \mid \alpha_{I} \neq 0\right\} .
$$


For $f=\Sigma_{I} \alpha_{I} M_{I} \in \mathbb{C}\left\{x_{1}, \ldots, x_{n}\right\}$ and $l \in \mathbb{Q}$, we define

$$
f_{\sigma-w t=l}=\sum_{\sigma-w t\left(M_{I}\right)=l} \alpha_{I} M_{I}
$$

For a rational number $k$, we also define

$$
I^{\sigma}(k)=\left\{f \in \mathbb{C}\left\{x_{1}, \ldots, x_{n}\right\} \mid \sigma-w t(f) \geq k\right\}
$$

which is an ideal of $\mathbb{C}\left\{x_{1}, \ldots, x_{n}\right\}$.

The following is immediate from (3.2) and the above definitions :

3.5. Lemma. Let $\bar{\pi}: \bar{Y} \rightarrow Y=\left(x_{1}, \ldots, x_{n}\right) / \mathbb{Z}_{m}$ be $a \sigma$-blow up and let $D$ be the $\mathbb{Q}$-Cartier Weil divisor defined by a $\mathbb{Z}_{m}$-semi-invariant $f \in \mathbb{C}\left\{x_{1}, \ldots, x_{n}\right\}$. Then we have

$$
\bar{\pi}^{*}(D)=\bar{\pi}^{-1}[D]+(\sigma-w t(f)) \bar{E},
$$

where $\bar{E}$ is the exceptional divisor of $\bar{\pi}$ and $\bar{\pi}^{-1}[D]$ is the proper transform of $D$.

From now on until the end of this section, we denote by $X$ a germ of a 3dimensional terminal singularitiy of index $m \geq 2$ and assume that the canonical cover of $X$ is not smooth.

3.6. Let $j: X \hookrightarrow(x, y, z, u) / \mathbb{Z}_{m}(\alpha, \beta, \gamma, \delta)$ be the standard embedding of $X$ as in (2.3). We shall say that the embedding $j^{\prime}: X \hookrightarrow\left(x^{\prime}, y^{\prime}, z^{\prime}, u^{\prime}\right) / \mathbb{Z}_{m}(\alpha, \beta, \gamma, \delta)$ is liftable if there is a $\mathbb{Z}_{m}$-equivariant automorphism $\bar{\chi}:(x, y, z, u) \rightarrow\left(x^{\prime}, y^{\prime}, z^{\prime}, u^{\prime}\right)$ such that $\chi \circ j=j^{\prime}$ where $\chi:(x, y, z, u) / \mathbb{Z}_{m} \rightarrow\left(x^{\prime}, y^{\prime}, z^{\prime}, u^{\prime}\right) / \mathbb{Z}_{m}$ is the automorphism induced by $\bar{\chi}$. Such an automorphism $\chi$ is called a liftable automorphism and we sometimes identify this with $\bar{\chi}$ if there would be no confusion.

Since all the automorphisms $\chi$ of $\mathbb{C}^{4} / \mathbb{Z}_{m}$ and all the embeddings $j^{\prime}: X \hookrightarrow\left(x^{\prime}\right.$, $\left.y^{\prime}, z^{\prime}, u^{\prime}\right) / \mathbb{Z}_{m}$ are liftable in this paper, we shall often omit the word "liftable" and just call automorphisms and embeddings respectively.

If $j^{\prime}: X \hookrightarrow\left(x^{\prime}, y^{\prime}, z^{\prime}, u^{\prime}\right) / \mathbb{Z}_{m}$ is an embedding, then the canonical cover of $X$ is a hypersurface in $\left(x^{\prime}, y^{\prime}, z^{\prime}, u^{\prime}\right)$. The defining equation of this hypersurface is called the defining equation of $j^{\prime}$. Thus we see that if $\varphi$ is a defining equation of $j$, then $\left(\chi^{-1}\right) *(\varphi)$ is a defining equation of $j^{\prime}$.

3.7. Weighted valuations. By a pseudo weighted valuation $v^{\prime}$, we mean a pair consisting of a liftable embedding $j^{\prime}: X \hookrightarrow\left(x^{\prime}, y^{\prime}, z^{\prime}, u^{\prime}\right) / \mathbb{Z}_{m}$ and a weight $\sigma^{\prime}=$ $\frac{1}{m}(a, b, c, d)$. We denote it by $v^{\prime}=\left(j^{\prime}, \sigma^{\prime}\right)$. This defines a weighted blow up of $X$ as in (3.2) and (3.3). We call this blow up the weighted blow up associated to $v^{\prime}$ or the $v^{\prime}$-blow up. If the $v^{\prime}$-blow up has an irreducible exceptional divisor, then this 
divisor determines a valuation on the function field of $X$, which is equivalent to the one determined by $\sigma^{\prime}-w t$. In this case, we call $v^{\prime}$ the weighted valuation. In general, the exceptional divisor may not be irreducible, so we call $v^{\prime}$ the pseudo weighted valuation.

Let $v^{\prime}=\left(j^{\prime}, \sigma^{\prime}\right)$ and $v^{\prime \prime}=\left(j^{\prime \prime}, \sigma^{\prime \prime}\right)$ be two pseudo weighted valuations. We define $v^{\prime}\left\langle v^{\prime \prime}\right.$ if for all liftable automorphism $\chi:\left(x^{\prime \prime}, y^{\prime \prime}, z^{\prime \prime}, u^{\prime \prime}\right) / \mathbb{Z}_{m} \rightarrow\left(x^{\prime}, y^{\prime}, z^{\prime}\right.$, $\left.u^{\prime}\right) / \mathbb{Z}_{m}$ such that $\chi \circ j^{\prime \prime}=j^{\prime}$ and for all $\mathbb{Z}_{m}$-semi-invariant $f \in \mathbb{C}\left\{x^{\prime}, y^{\prime}, z^{\prime}, u^{\prime}\right\}$, the inequalities $\sigma^{\prime}-w t(f) \leq \sigma^{\prime \prime}-w t\left(\chi^{*} f\right)$ hold.

This relation defines a pseudo order on the set of pseudo weighted valuations. Hence the relation $v^{\prime} \sim v^{\prime \prime}$ defined by $v^{\prime}<v^{\prime \prime}$ and $v^{\prime \prime}<v^{\prime}$ gives an equivalence relation.

3.8. Lemma. Let $v^{\prime}=\left(j^{\prime}, \sigma^{\prime}\right)$ and $v^{\prime \prime}=\left(j^{\prime \prime}, \sigma^{\prime \prime}\right)$ be two pseudo weighted valuations. Then the following ( $\mathrm{i}$ ) and (ii) are equivalent :

(i) $v^{\prime}<v^{\prime \prime}$.

(ii) For all liftable automorphism $\chi:\left(x^{\prime \prime}, y^{\prime \prime}, z^{\prime \prime}, u^{\prime \prime}\right) / \mathbb{Z}_{m} \rightarrow\left(x^{\prime}, y^{\prime}, z^{\prime}, u^{\prime}\right) / \mathbb{Z}_{m}$ with $\chi \circ j^{\prime \prime}=j^{\prime}$, we have

$$
\begin{aligned}
& \sigma^{\prime \prime}-w t\left(\chi^{*}\left(x^{\prime}\right)\right) \geq \sigma^{\prime}-w t\left(x^{\prime}\right), \quad \sigma^{\prime \prime}-w t\left(\chi^{*}\left(y^{\prime}\right)\right) \geq \sigma^{\prime}-w t\left(y^{\prime}\right), \\
& \sigma^{\prime \prime}-w t\left(\chi^{*}\left(z^{\prime}\right)\right) \geq \sigma^{\prime}-w t\left(z^{\prime}\right) \text { and } \sigma^{\prime \prime}-w t\left(\chi^{*}\left(u^{\prime}\right)\right) \geq \sigma^{\prime}-w t\left(u^{\prime}\right) .
\end{aligned}
$$

We further assume that $\sigma^{\prime \prime}-w t\left(x^{\prime \prime}\right), \ldots, \sigma^{\prime \prime}-w t\left(u^{\prime \prime}\right) \leq \sigma^{\prime \prime}-w t\left(\varphi^{\prime \prime}\right)$ where $\varphi^{\prime \prime}$ is the defining equation of $j^{\prime \prime}$. Then ( $\mathrm{i}$ ) and (ii) are also equivalent to the following :

(iii) For some liftable automorphism $\chi:\left(x^{\prime \prime}, y^{\prime \prime}, z^{\prime \prime}, u^{\prime \prime}\right) / \mathbb{Z}_{m} \rightarrow\left(x^{\prime}, y^{\prime}, z^{\prime}, u^{\prime}\right) /$ $\mathbb{Z}_{m}$ with $\chi \circ j^{\prime \prime}=j^{\prime}$, the inequalities (3.8.1) holds.

Proof. It is enough to prove that (iii) implies (ii). Let $\chi_{i}:\left(x^{\prime \prime}, y^{\prime \prime}, z^{\prime \prime}, u^{\prime \prime}\right) /$ $\mathbb{Z}_{m} \rightarrow\left(x^{\prime}, y^{\prime}, z^{\prime}, u^{\prime}\right) / \mathbb{Z}_{m}(i=1,2)$ be liftable automorphisms satisfying $\chi_{\imath} \circ j^{\prime \prime}=j^{\prime}$. Then we have $\chi_{1}^{*}\left(x^{\prime}\right)-\chi_{2}^{*}\left(x^{\prime}\right) \in \varphi^{\prime \prime} \mathbb{C}\left\{x^{\prime \prime}, y^{\prime \prime}, z^{\prime \prime}, u^{\prime \prime}\right\}$. By our assumption, $\sigma^{\prime \prime}-w t\left(\chi_{i}^{*}\left(x^{\prime}\right)\right)<\sigma^{\prime \prime}-w t\left(\varphi^{\prime \prime}\right)$. Thus we see that $\sigma^{\prime \prime}-w t\left(\chi_{1}^{*}\left(x^{\prime}\right)\right)=\sigma^{\prime \prime}-w t\left(\chi_{2}^{*}\left(x^{\prime}\right)\right)$. This equality holds if we replace $x^{\prime}$ by $y^{\prime}, z^{\prime}$ or $u^{\prime}$. Therefore we see that (iii) implies (ii).

We shall often abbreviate the condition (3.8.1) as

$$
\sigma^{\prime \prime}-w t s\left(\chi^{*}\left(x^{\prime}, y^{\prime}, z^{\prime}, u^{\prime}\right)\right) \geq \sigma^{\prime}-w t s\left(x^{\prime}, y^{\prime}, z^{\prime}, u^{\prime}\right)
$$

3.9. Discrepancies. Let $v^{\prime}=\left(j^{\prime}, \sigma^{\prime}\right)$ be a pseudo weighted valuation and let $\sigma^{\prime}=\frac{1}{m}(a, b, c, d)$. Then we constructed the $\sigma^{\prime}$-blow up $\bar{\pi}: \bar{Y} \longrightarrow Y=\left(x^{\prime}, y^{\prime}, z^{\prime}\right.$, $\left.u^{\prime}\right) / \mathbb{Z}_{m}$ and the $v^{\prime}$-blow up $\pi: \bar{X} \longrightarrow X$ (cf. (3.2), (3.3)). Let $\bar{E}$ denote the exceptional divisor of $\bar{\pi}$. By using the theory of toric varieties or by direct calculations using (3.2.2), we have 


$$
\begin{gathered}
K_{\bar{Y}}=\bar{\pi}^{*} K_{Y}+\frac{1}{m}(a+b+c+d-m) \bar{E} \\
K_{\bar{X}}=\pi^{*} K_{X}+\left(w\left(x^{\prime}\right)+w\left(y^{\prime}\right)+w\left(z^{\prime}\right)+w\left(u^{\prime}\right)-w\left(\varphi^{\prime}\right)-1\right)\left(\left.\bar{E}\right|_{\bar{X}}\right)
\end{gathered}
$$

where $\varphi^{\prime}$ is the defining equation of $j^{\prime}: X \hookrightarrow\left(x^{\prime}, y^{\prime}, z^{\prime}, u^{\prime}\right) / \mathbb{Z}_{m}$ and $w=\sigma^{\prime}-w t$.

We define

$$
\begin{aligned}
d\left(v^{\prime}\right) & =w\left(x^{\prime}\right)+w\left(y^{\prime}\right)+w\left(z^{\prime}\right)+w\left(u^{\prime}\right)-w\left(\varphi^{\prime}\right)-1 \\
& =\frac{1}{m}(a+b+c+d)-w\left(\varphi^{\prime}\right)-1,
\end{aligned}
$$

and call it the virtual discrepancy of $v^{\prime}$. If $\left.\bar{E}\right|_{\bar{X}}$ is irreducible and reduced, then we have $d\left(v^{\prime}\right)=a\left(\left.\bar{E}\right|_{\bar{X}}, X\right)($ cf. (2.7)).

3.10. For a positive rational number $\alpha$, we define

$$
\mathscr{W}_{\alpha}=\left\{\begin{array}{l|l}
v^{\prime}=\left(j^{\prime}, \sigma^{\prime}\right) & \begin{array}{l}
j^{\prime}: X \hookrightarrow\left(x^{\prime}, y^{\prime}, z^{\prime}, u^{\prime}\right) / \mathbb{Z}_{m}: \text { liftable embedding, } \\
\sigma^{\prime}: \text { weight, } \quad d\left(v^{\prime}\right)=\alpha
\end{array}
\end{array}\right\} .
$$

When we fix a liftable embedding $j^{\prime}: X \hookrightarrow\left(x^{\prime}, y^{\prime}, z^{\prime}, u^{\prime}\right) / \mathbb{Z}_{m}$, the subset of $\mathscr{W}_{\alpha}$ with fixed embedding $j^{\prime}$ is denoted by $\mathscr{W}_{\alpha}\left(j^{\prime}\right)$.

The relation $<$ and $\sim$ in (3.7) also define a pseudo order and an equivalence relation on $\mathscr{W}_{\alpha}$ respectively. So the relation $\prec$ defines an order on $\mathscr{W}_{\alpha} / \sim$.

One of our main objects to study is the maximal elements in $\mathscr{W}_{1 / m} / \sim$ where $m$ is the index of $X$.

\section{§ 4. Main Results and Comments on the Proof}

In this section, we shall summarize the main results obtained in this paper and give some comments on the proof. Our main result is the following :

4.1. Theorem. Let $X$ be a germ of a 3-dimensional terminal singularity of index $m \geq 2$, and assume that $X$ is neither a cyclic quotient singularity nor of type (cD/2). Then the following holds :

(1) If $v \in \mathscr{W}_{1 / m}$ is maximal with respect to $\prec$, then the $v$-blow up of $X$ is divisorial with discrepancy $1 / \mathrm{m}$.

(2) For each divisorial blow up $\pi: \bar{X} \rightarrow X$ with discrepancy $1 / m$, there are some $v \in \mathscr{W}_{1 / m}$ such that $\pi$ is isomorphic to the $v$-blow up of $X$.

(3) There is a one-to-one correspondence between the set of all maximal elements 
of $\mathscr{W}_{1 / m} / \sim$ and the set of all isomorphism classes of divisorial blow ups of $X$ with discrepancies $1 / \mathrm{m}$.

4.2. In order to prove (4.1), we proceed as follows :

First we determine all the possible weights $\sigma^{\prime}$ such that $\left(j^{\prime}, \sigma^{\prime}\right) \in \mathscr{W}_{1 / m}$ for some embedding $j^{\prime}: X \hookrightarrow\left(x^{\prime}, y^{\prime}, z^{\prime}, u^{\prime}\right) / \mathbb{Z}_{m}$. Since $X$ is terminal, the defining equation of $j^{\prime}$ must have some lower degree terms, which restricts the possible weights. We fix one embedding $j_{1}: X \hookrightarrow\left(x_{1}, y_{1}, z_{1}, u_{1}\right) / \mathbb{Z}_{m}$ and determine $\mathscr{W}_{1 / m}\left(j_{1}\right)$ and maximal elements of $\mathscr{W}_{1 / m}\left(j_{1}\right)$. This is immediate from the discussion just above.

Let $v=\left(j_{1}, \sigma\right) \in \mathscr{W}_{1 / m}\left(j_{1}\right)$ and let $\pi: \bar{X} \rightarrow X$ be the $v$-blow up of $X$. We shall study singularities of $\bar{X}$ and the exceptional divisor of $\pi$. This calculation will be done by using the description given in (3.2).

If the exceptional divisor of $\pi$ is reducible or if $\bar{X}$ has non-terminal singularities, then closer analysis of these naturally leads to another pseudo weighted valuation $v^{\prime}>v$. We repeat the process starting with new pseudo weighted valuation $v^{\prime}$. This process terminates by the "boundedness" of weights.

Let $v_{1}, \ldots, v_{k}$ be all the pseudo weighted valuations which give divisorial blow ups of $X$ with discrepancies $1 / m$ by the above procedure and assume that these $v_{i}$-blow ups of $X$ are not mutually isomorphic.

Next, we shall prove that for every maximal element $v^{\prime} \in \mathscr{W}_{1 / m}, v^{\prime}>v_{i}$ for some $i$. Since the $v_{i}$-blow up is divisorial, it follows from (5.8) that $v^{\prime} \sim v_{i}$. Thus we know that $v_{1}, \ldots, v_{k}$ represent all the maximal elements in $\mathscr{W}_{1 / m} / \sim$. Thus we can prove (4.1)(1).

Lastly we count the number of divisors with discrepancies $1 / \mathrm{m}$. Starting with one divisorial blow up $\pi: \bar{X} \rightarrow X$ obtained earlier, we shall make a partial resolution of $\nu: Z \rightarrow \bar{X}$ so that all divisors with discrepancies $1 / m$ over $X$ appear as divisors on $Z$. Such divisors may have discrepancies $\neq 1 / m$ over $\bar{X}$, however these can be determined by case by case analysis. These calculations will be done by using ( 5.1$)$ and (5.3).

Let $d_{1}$ be the number of non-isomorphic divisorial blow ups of $X$ with discrepancies $1 / m$ and let $d_{2}$ be the number of divisors with discrepancies $1 / m$ over $X$. In general, we have $d_{1} \leq d_{2}$.

If $d_{1}=d_{2}$ (this always holds if $X$ is of type $(\mathrm{cA} / \mathrm{m}),(\mathrm{cAx} / 4)$ and $\left.(\mathrm{cAx} / 2)\right)$, then (4.1) (2) will be proved by using (5.7).

If $d_{1}<d_{2}$ (this occurs only if $X$ is of type $(\mathrm{cD} / 3)$ and $(\mathrm{cE} / 2)$ ), then we shall again look for the blow up extracting the divisor with discrepancy $1 / \mathrm{m}$. In all the cases these are obtained by the $v^{\prime}$-blow up for $v^{\prime} \in \mathscr{W}_{1 / m}$ which are not maximal.

Thus for each divisorial blow up $\pi: \bar{X} \rightarrow X$ with discrepancy $1 / m$, we can find the maximal element $v$ in $\mathscr{W}_{1 / m}$ such that the $v$-blow up of $X$ is isomorphic to $\pi$, and this will complete the proof of (4.1)(2).

If $v, v^{\prime} \in \mathscr{W}_{1 / m}$ satisfy $v \sim v^{\prime}$, then the $v$-blow up and $v^{\prime}$-blow up of $X$ is 
isomorphic by (5.6). Hence (4.1) (3) is an easy conseqence of (4.1) (1) and (4.1) (2).

4.3. Remark. If $X$ is a cyclic quotient terminal singularity of index $m \geq 2$, then $\mathscr{W}_{1 / m} / \sim$ contains infinitely many elements and we can not determine the maximal elements of $\mathscr{W}_{1 / m}$. In this case, there is a nice resolution of $X$ as described in (5.1) and all the divisorial blow ups of $X$ is determined by [Kaw96].

If $X$ is terminal of type (cD/2), then (4.1)(2) and (4.1) (3) are not true in some cases. Indeed, if $X$ is of type (cD/2-1) and assume that $a=2, b \geq 3$ in (2.3), then we can see that $\mathscr{W}_{1 / 2} / \sim$ has only one maximal element, however $X$ has 2 divisors with discrepancies $1 / 2$ and both of them can be obtained as exceptional divisors of some divisorial blow ups with discrepancies $1 / 2$. If $X$ is of type (cD/ $2-2$ ), the situation is more complicated. The details of these will be analysed in our future paper.

The following three corollaries will be obtained in the course of the proof of our main theorem. These include the case where $X$ is a cyclic quotient terminal singularity. In this case, proofs of these colollaries are given at the end of this section.

4.4. Corollary。 Let $X$ be a germ of a 3-dimensional terminal singularity of index $m \geq 2$. Assume that $X$ is not of type $(\mathrm{cD} / 2)$. Then there is at least one divisorial blow up of $X$ with discrepancy $1 / \mathrm{m}$. Moreover, for each divisor $E$ over $X$ with discrepancy $1 / m$, there is a $v=(j, \sigma) \in \mathscr{W}_{1 / m}$ such that the $v$-blow up $\pi: \bar{X} \rightarrow$ $X$ satisfies the following :

(i ) $\bar{X}$ has only canonical singularities, and

(ii) $E$ is the exceptional divisor of $\pi$.

4.5. Corollary. Let $X$ be a germ of a 3-dimensional terminal singularity at $P$ $\in X$. Assume that the index $m$ of $X$ at $P$ is $\geq 2$ and that $X$ is not of type (cD/2). Let $\pi: \bar{X} \rightarrow X$ be an arbitrary divisorial blow up with discrepancy $1 / m$. Then we have

$$
\sum_{Q \in \bar{X}}(\operatorname{aw}(\bar{X}, Q)-1) \leq \operatorname{aw}(X, P)-1 .
$$

The equality holds only if $X$ is a cyclic quotient singualrity or of type (cD/3).

4.6. Corollary. Let $X$ be a germ of a 3-dimensional terminal singularity of index $m \geq 2$. Let $n$ be the number of divisors of discrepancies $1 / m$. Using the notation of (2.3), we have the following :

$(\mathrm{cA} / \mathrm{m})$ Let $\tau-w t(z)=1 / m, \tau-w t(u)=1$. Then $n=\tau$-wt $(f(z, u))$.

$(\mathrm{cAx} / 4)$ Let $\tau$-wt $(z)=1 / 4, \tau$-wt $(u)=1 / 2$ and assume that $\tau-w t(f(z, u))=(2 k+$ 1)/2. Then, 
(i ) $n=1$ if $f_{\tau-w t=(2 k+1) / 2}(z, u)$ is not a square,

(ii) $n=2$ otherwise.

$(\mathrm{cAx} / 2)$ Let $\tau-w t(z)=\tau-w t(u)=1 / 2$ and assume that $\tau-w t(f(z, u))=k$. Then,

(i ) $n=1$ if $f_{\tau-w t=k}(z, u)$ is not a square,

(ii) $n=2$ otherwise.

(cD/3-1) $n=3$.

(cD/3-2) $n=2$.

(cD/3-3) We denote the power series expansion of $\alpha\left(z^{3}\right)$ etc. by $\alpha\left(z^{3}\right)=\sigma_{0}+\alpha_{1} z^{3}+$ $\alpha_{2} z^{6}+\cdots$, etc. Consider the system of equations

$$
x^{3}+\beta_{0} x+\delta_{0}=0, \quad 3 x^{2}+\beta_{0}=0, \quad \alpha_{0} x+\gamma_{0}=0, \quad \beta_{1} x+\delta_{1}=0
$$

in $x$. Then,

(i ) $n=1$ if (*) has no solutions,

(ii) $n=2$ if (*) has a solution.

(cE/2) We denote the degree 4 part of $h(y, z)$ by $h_{\operatorname{deg} 4}(y, z)$ and the power series expansion of $g(y, z)$ and $h(y, z)$ by $g(y, z)=\sum_{i, j} a_{i, j} y^{i} z^{j}$ and $h(y, z)=\sum_{j, j} b_{i, j} y^{i} z^{j}$ respectively.

(i) If $h_{\operatorname{deg}} 4(y, z)$ does not have a triple or a 4-ple factor, then $n=$ the number of distinct factors of $h_{\operatorname{deg}} 4(y, z)$. In particular, $n \leq 4$.

(ii) If $h_{\operatorname{deg} 4}(y, z)$ has a triple factor and a single factor, and if we assume $h_{\operatorname{deg} 4}(y, z)=y^{3} z$, consider the system of equations

$$
x^{3}+a_{0,4} x+b_{0,6}=0, \quad 3 x^{2}+b_{0,6}=0, \quad a_{0,6} x+b_{0,8}=0, \quad a_{1,3} x+b_{1,5}=0
$$

in $x$. Then,

(ii - i ) $n=2$ if ( $\dagger$ ) has no solutions,

(ii-ii) $n=3$ if ( $\dagger$ ) has a solution.

(iii) If $h_{\operatorname{deg} 4}(y, z)$ has a 4-ple factor, and if we assume $h_{\operatorname{deg} 4}(y, z)=y^{4}$, we first consider the above system of equations $(\dagger)$.

(iii - i ) $n=1$ if ( $\dagger$ ) has no solutions.

If $(\dagger)$ has a solution, there is an embedding $j_{1}$ of $X$ such that

$$
\begin{gathered}
j_{1}: X \simeq\left\{u_{1}^{2}+x_{1}^{3}+\lambda x_{1}^{2} z_{1}^{2}+g_{1}\left(y_{1}, z_{1}\right) x_{1}+h_{1}\left(y_{1}, z_{1}\right)=0\right\} / \mathbb{Z}_{2}(0,1,1,1) \\
\subseteq\left(x_{1}, y_{1}, z_{1}, u_{1}\right) / \mathbb{Z}_{2}(0,1,1,1)
\end{gathered}
$$

where $\lambda \in \mathbb{C}, \tau$-wt $\left(g_{1}(y, z)\right) \geq 3, \tau$-wt $\left(h_{1}(y, z)\right) \geq 5$ when we set $\tau$-wt $(y)=3 / 2$ and $\tau$-wt $(u)=1 / 2$. Now we consider

$$
\Phi=u_{1}^{2}+\lambda x_{1}^{2} z_{1}^{2}+g_{1, \tau-w t=3}\left(y_{1}, z_{1}\right) x_{1}+h_{1, \tau-w t=5}\left(y_{1}, z_{1}\right) .
$$


Then,

(iii - ii) $n=2$ if $\Phi$ is irreducible and reduced,

(iii - iii) $n=3$ if $\Phi$ has 2 distinct irreducible components.

(iii-iv) $n=2$ if $\Phi=u_{1}^{2}$ and the $\left(j_{1}, \frac{1}{2}(4,3,1,7)\right)$-blow up of $X$ is divisorial,

(iii-v ) $n=3$ if $\Phi=u_{1}^{2}$ and the $\left(j_{1}, \frac{1}{2}(4,3,1,7)\right)$-blow up of $X$ is not divisorial.

If $X=(x, y, z) / \mathbb{Z}_{m}(\alpha,-\alpha, 1)$ is a cyclic quotient terminal singularitiy, then (4.5) and (4.6) hold by [Kaw96] and (5.1). We define the embedding

$$
j: X \simeq\{u=0\} / \mathbb{Z}_{m}(\alpha,-\alpha, 1,0) \subseteq(x, y, z, u) / \mathbb{Z}_{m}(\alpha,-\alpha, 1,0)
$$

and a weight $\sigma=(\langle\alpha / m\rangle,\langle-\alpha / m\rangle, 1 / m, 1)$. We denote $v=(j, \sigma)$. Then the $v$ blow up of $X$ extracts the unique divisor with discrepancy $1 / m$ over $X$. This proves (4.4) if $X$ is a cyclic quotient terminal singularity. We shall omit cyclic quotient singularities when we consider terminal singularities of type $(\mathrm{cA} / \mathrm{m})$.

Many parts of these corollaries have counterparts in the $(\mathrm{cD} / 2)$ case. For instance, (4.5) also holds and we can calculate the number of divisors with discrepancy $1 / 2$ for terminal singularities of type $(\mathrm{cD} / 2)$. These also will be treated in our future paper.

\section{§ 5. Some Auxiliary Results}

In this section, we shall collect some results which will be used in the following sections.

The following proposition is due to Danilov and Barlow (see [Reid87, (5.7)]) which shows the existence of economic resolutions of cyclic quotient terminal singularities.

5.1. Proposition. Let $X=(x, y, z) / \mathbb{Z}_{m}(\alpha,-\alpha, 1)$ ( $\alpha$ is prime to $m$ ) be the germ of a cyclic quotient terminal singularity of index $m \geq 2$. Then there is $a$ projective birational morphism $\nu: Z \rightarrow X$ such that

(i) $Z$ is non-singular,

(ii) $K_{Z}=\nu^{*}\left(K_{X}\right)+\sum_{i=1}^{m-1} \frac{i}{m} F_{i}$, where $\sum_{i=1}^{m-1} F_{i}$ is the exceptional divisor of $\nu$. Furthermore, if $D$ is a $\mathbb{Q}$-Cartier Weil divisor on $X$ defined by a $\mathbb{Z}_{m}$-semi-invariant $f(x, y, z) \in \mathbb{C}\{x, y, z\}$, then

(iii) $\nu^{*}(D)=\nu^{-1}[D]+\sum_{i=1}^{m-1} d_{i} F_{i}$, where $\nu^{-1}[D]$ denotes the proper transform of $D$ by $\nu$, and $d_{i}=\sigma_{i}-w t(f(x, y, z)), \sigma_{i}=(\langle\alpha i / m\rangle,\langle-\alpha i / m\rangle, i / m)$.

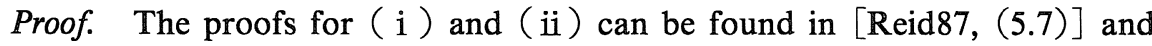
(iii) follows from (3.5). 
5.2. Corollary. Let $X$ be a germ of a 3-dimensional cyclic quotient terminal singularity of index $m \geq 2$. Then there is a unique divisor with discrepancy $i / m$ over $X$ for each $i=1,2, \ldots, m-1$.

The following will be used to estimate the number of divisors with minimal discrepancies :

5.3. Proposition. Let $X$ be a germ of a 3-dimensional terminal singularity of index $m \geq 2$. Let $\pi: \bar{X} \rightarrow X$ be a divisorial blow up with discrepancy $1 / m$ and let $E$ be the exceptional divisor of $\pi$. Let $\nu: Z \rightarrow \bar{X}$ be a partial resolution of $\bar{X}$ and $\Sigma F_{i}$ be the exceptional divisor of $\nu$. If $\nu^{*}(E)=\nu^{-1}[E]+\sum a_{i} F_{i}$, then we have

$$
a\left(F_{i}, X\right)=a\left(F_{i}, \bar{X}\right)+a_{i} / m
$$

for each $i$. In particular, if $Q \in \bar{X}$ is of index $\leq m$ and $Q \in E$, then there are no prime divisors over $Q$ with discrepancies $1 / m$ over $X$.

Proof. The first part follows by comparing $K_{Z}$ with $\nu^{*}\left(\pi^{*}\left(K_{X}\right)\right)$. The second part is easily deduced from the first part.

Next, we shall show some lemmas which will be used to determine the maximal elements in $\mathscr{W}_{1 / m}$. In the following lemmas (5.4)-(5.8), $X$ denotes a germ of a 3dimensional terminal singularity of index $m \geq 2$ and assume that the canonical cover of $X$ is not smooth.

5.4. Lemma. Let $v=(j, \sigma), v^{\prime}=\left(j^{\prime}, \sigma^{\prime}\right) \in \mathscr{W}_{1 / m}$ and assume that $\sigma=\sigma^{\prime}$. If $\sigma=\frac{1}{m}(a, b, c, d)$ satisfisfies $0<a, b, c, d \leq m$, then we have $v \sim v^{\prime}$.

Proof. Let $\chi:\left(x^{\prime}, y^{\prime}, z^{\prime}, u^{\prime}\right) / \mathbb{Z}_{m} \rightarrow(x, y, z, u) \mathbb{Z}_{m}$ be a liftable automorphism such that $\chi \circ j^{\prime}=j$. Since $\chi$ comes from a $\mathbb{Z}_{m}$-equivariant automorphism of $\mathbb{C}^{4}$, we have

$$
\sigma^{\prime}-w t s\left(\chi^{*}(x, y, z, u)\right) \equiv \frac{1}{m}(a, b, c, d) \quad \bmod \mathbb{Z}^{4}
$$

Thus our assumption implies that

$$
\sigma^{\prime}-w t s\left(\chi^{*}(x, y, z, u)\right) \geq \frac{1}{m}(a, b, c, d)=\sigma-w t s(x, y, z, u) .
$$

This shows that $v^{\prime}>v$. Similarly, we can prove $v^{\prime}<v$.

5.5. Lemma. Let $v=(j, \sigma), v^{\prime}=\left(j^{\prime}, \sigma^{\prime}\right) \in \mathscr{W}_{1 / m}$ and assume that $\sigma=\sigma^{\prime}$ and $v<v^{\prime}$. Then we have $v \sim v^{\prime}$. 
Proof. Let $\chi:\left(x^{\prime}, y^{\prime}, z^{\prime}, u^{\prime}\right) / \mathbb{Z}_{m} \rightarrow(x, y, z, u) / \mathbb{Z}_{m}$ be a liftable automorphism such that $\chi \circ j^{\prime}=j$ and let $f(x, y, z, u) \in \mathbb{C}\{x, y, z, u\}$ be a $\mathbb{Z}_{m}$-semi-invariant. We assume that $\sigma$-wt $(f)=l$ and write $f=f_{\sigma-w t=l}+f_{\sigma-w t>l}$. Since $v<v^{\prime}$, we have $\sigma^{\prime}-w t\left(\chi^{*}(f)\right) \geq l$. Let $f_{1}$ be the lowest degree part of $f_{\sigma-w t=l}$. Since $\chi$ comes from an automorphism of $\mathbb{C}^{4}, \chi^{*}\left(f_{1}\right)$ does not vanish and $\sigma^{\prime}-w t\left(\chi^{*}\left(f_{1}\right)\right)=\sigma-w t\left(f_{1}\right)=l$. Hence $\sigma^{\prime}-w t\left(\chi^{*}(f)\right)=l$ and we know that $v \sim v^{\prime}$.

5.6. Lemma. Let $v, v^{\prime} \in \mathscr{W}_{1 / m}$ satisfy $v \sim v^{\prime}$. Then the $v$-blow up of $X$ and $v^{\prime}-$ blow up of $X$ are isomorphic.

Proof. This is obvious since blowing up ideals coincide.

The following proposition will be used to prove our main results and it is proved in [FA92, 6.2] and [Luo98, 2.4].

5.7. Proposition. Let $\pi: \bar{X} \rightarrow X$ (resp. $\pi^{\prime}: \bar{X}^{\prime} \rightarrow X$ ) be a divisorial blow up with exceptional divisor $E$ (resp. $E^{\prime}$ ). If $E$ and $E^{\prime}$ define the same valuation on the function field $\mathbb{C}(X)$ of $X$, then $\bar{X}$ and $\bar{X}^{\prime}$ are isomorphic over $X$.

Proof. Since $K_{\bar{X}}=\pi^{*}\left(K_{X}\right)+\alpha E$ for some $\alpha>0$, we see that $E$ is $\mathbb{Q}$-Cartier and $-E$ is $\pi$-ample. Similarly, $E^{\prime}$ is also $\mathbb{Q}$-Cartier and $-E^{\prime}$ is $\pi^{\prime}$-ample. By our assumption, $E$ corresponds to $E^{\prime}$ by the rational map $\pi^{\prime-1} \circ \pi$. Thus we get that

$$
\bar{X} \simeq \operatorname{Proj}\left(\bigoplus_{\imath \geq 0} \pi_{*}(-i E)\right) \simeq \operatorname{Proj}\left(\bigoplus_{i \geq 0} \pi_{*}^{\prime}\left(-i E^{\prime}\right)\right) \simeq \bar{X}^{\prime} .
$$

The following lemma easily follows from [Kaw96] and will also be used in the proof of our main results :

5.8. Lemma. Let $v=(j, \sigma), v^{\prime}=\left(j^{\prime}, \sigma^{\prime}\right) \in \mathscr{W}_{1 / m}$ satisfy $v<v^{\prime}$. If the $v$-blow up of $X$ is divisorial with discrepancy $1 / m$, then we have $v \sim v^{\prime}$.

Proof. Let $\pi: \bar{X} \rightarrow X$ be the $v$-blow up and let $E$ be the exceptional divisor of $\pi$. Since $-E$ is $\pi$-ample, there is a general member $L \in|-l E|$ for a sufficient large and divisible $l$. Let $D=\pi_{*}(L)$. Since $D \sim 0, D$ is defined by a $\mathbb{Z}_{m}$-invariant $f(x, y$, $z, u)$. Then we have $\pi^{*}(D)=\pi^{-1}[D]+(\sigma-w t(f)) E$ by (3.5).

Let $\bar{\pi}^{\prime}: \bar{Y}^{\prime} \rightarrow\left(x^{\prime}, y^{\prime}, z^{\prime}, u^{\prime}\right) / \mathbb{Z}_{m}$ be the $\sigma^{\prime}$-blow up, let $\bar{E}^{\prime}$ be the exceptional divisor of $\bar{\pi}^{\prime}$ and let $\pi^{\prime}: \bar{X}^{\prime} \rightarrow X$ be the restriction of $\bar{\pi}^{\prime}$. By (3.5) and $d\left(v^{\prime}\right)=1 / m$, we have

$$
K_{\bar{X}^{\prime}}=\pi^{*} K_{X}+\frac{1}{m}\left(\left.\bar{E}^{\prime}\right|_{\bar{X}^{\prime}}\right) \text { and } \pi^{\prime *}(D)=\pi^{\prime-1}[D]+\left(\sigma^{\prime}-w t\left(\chi^{*}(f)\right)\right)\left(\left.\bar{E}^{\prime}\right|_{\bar{X}^{\prime}}\right)
$$

where $\chi:\left(x^{\prime}, y^{\prime}, z^{\prime}, u^{\prime}\right) / \mathbb{Z}_{m} \rightarrow(x, y, z, u) / \mathbb{Z}_{m}$ is a liftable automorphism such that 
$\chi \circ j^{\prime}=j$

Let $\nu: Z \rightarrow \bar{X}$ and $\nu^{\prime}: Z \rightarrow \bar{X}^{\prime}$ be common resolutions and let $\Sigma F_{j}$ be the exceptional diviosr of $\nu$.

Since $\bar{X}$ has only terminal singularities, we have $K_{Z}=\nu^{*}\left(K_{\bar{X}}\right)+\sum a_{j} F_{j}$ with all $a_{j}>0$. So we see that $K_{Z}=\nu^{*} \pi^{*}\left(K_{X}\right)+\frac{1}{m}\left(\nu^{*}(E)+\sum m a_{j} F_{j}\right)$ and therefore we get $\left.\bar{E}^{\prime}\right|_{\bar{X}^{\prime}}=\nu_{*}^{\prime}\left(\nu^{*}(E)+\sum m a_{j} F_{j}\right)$. On the other hand, we have $\pi^{\prime *}(D)=\pi^{\prime-1}[D]+$ $(\sigma-w t(f)) \nu_{*}^{\prime} \nu^{*}(E)$ since $L=\pi^{-1}[D]$ is general. Thus we know that

$$
\left(\sigma-w t(f)-\sigma-w t\left(\chi^{*}(f)\right)\right) \nu_{*}^{\prime} \nu^{*}(E)=\left(\sigma^{\prime}-w t\left(\chi^{*}(f)\right)\right) \nu_{*}^{\prime}\left(\sum m a_{j} F_{j}\right)
$$

Since $v^{\prime}>v$, we have $\sigma$-wt $(f) \leq \sigma^{\prime}-w t\left(\chi^{*}(f)\right)$ so that we have $\nu_{*}^{\prime}\left(\sum m a_{j} F_{j}\right)=0$. Since all the $a_{j}>0$, we see that $\left.\bar{E}^{\prime}\right|_{\bar{X}^{\prime}}$ is irreducible and reduced. We also see that $\left.\bar{E}^{\prime}\right|_{\bar{X}^{\prime}}$ and $E$ define the same valuation on the function field of $X$. Therefore $\bar{X}$ and $\bar{X}^{\prime}$ are isomorphic over $X$ by (5.7).

Let $D_{x}$ be the $\mathbb{Q}$-Cartier Weil divisor on $X$ defined by $x=0$. Then we have

$$
\begin{aligned}
& \pi^{*}\left(D_{x}\right)=\pi^{-1}\left[D_{x}\right]+(\sigma-w t(x)) E \text { and } \\
& \pi^{*}\left(D_{x}\right)=\pi^{\prime-1}\left[D_{x}\right]+\left(\sigma^{\prime}-w t\left(\chi^{*}(x)\right)\right)\left(\left.\bar{E}\right|_{\bar{X}^{\prime}}\right),
\end{aligned}
$$

since the canonical cover of $X$ is not smooth. Since $\bar{X}$ and $\bar{X}^{\prime}$ are isomorphic, we see that $\sigma$-wt $(x)=\sigma^{\prime}-w t\left(\chi^{*}(x)\right)$. Thus $\sigma$-wt $(g)=\sigma^{\prime}-w t\left(\chi^{*}(g)\right)$ for all $\mathbb{Z}_{m}$-semiinvariant $g \in \mathbb{C}\{x, y, z, u\}$. This shows that $v \sim v^{\prime}$.

\section{$\S$ 6. Terminal Singularities of Type $(\mathbf{c A} / \mathrm{m})$}

6.1. Let $X$ be a germ of a 3-dimensional terminal singularity of type $(\mathrm{cA} / \mathrm{m})$ with $m \geq 2$. By (2.3), there is a standard embedding

$$
j: X \simeq\{x y+f(z, u)=0\} / \mathbb{Z}_{m}(\alpha,-\alpha, 1,0) \subseteq(x, y, z, u) / \mathbb{Z}_{m}(\alpha,-\alpha, 1,0)
$$

where $\alpha$ is prime to $m$ and $f(z, u)$ is a $\mathbb{Z}_{m}$-invariant. In this case, we have aw $(X)$ $=\operatorname{ord}(f(0, u))$. We denote the defining equation of $j$ as $\varphi=x y+f(z, u)$. We assume that $X$ is not a cyclic quotient singularity.

6.2. Lemma. Let $j^{\prime}: X \hookrightarrow\left(x^{\prime}, y^{\prime}, z^{\prime}, u^{\prime}\right) / \mathbb{Z}_{m}(\alpha,-\alpha, 1,0)$ be an arbitrary embedding and let $\varphi^{\prime}$ be the defining equation of $j^{\prime}$. Then, after a permutation of coordinates if necessary, we have
(1) $x^{\prime} y^{\prime} \in \varphi^{\prime}$ if $m \geq 3$.
(2) $x^{\prime 2}, y^{\prime 2} \in \varphi^{\prime}$ or $x^{\prime} y^{\prime} \in \varphi^{\prime}$ if $m=2$. 
Furthermore, if $\left(j^{\prime}, \sigma^{\prime}\right) \in \mathscr{W}_{1 / m}$, then $\sigma^{\prime}-w t\left(z^{\prime}\right)=1 / m, \sigma^{\prime}-w t\left(u^{\prime}\right)=1$.

Proof. Since $X$ is not a cyclic quotient singularity, $\varphi^{\prime}$ does not contain linear terms, so the first part follows. To prove the second part, we denote $w=\sigma^{\prime}-w t$. Since $x^{\prime} y^{\prime} \in \varphi^{\prime}$ or $x^{\prime 2}, y^{\prime 2} \in \varphi^{\prime}$, we have $w\left(x^{\prime}\right)+w\left(y^{\prime}\right) \geq w\left(\varphi^{\prime}\right)$. By (3.9.1), we see that

$$
1 / m=w\left(x^{\prime}\right)+w\left(y^{\prime}\right)+w\left(z^{\prime}\right)+w\left(u^{\prime}\right)-w\left(\varphi^{\prime}\right)-1 \geq w\left(z^{\prime}\right)+w\left(u^{\prime}\right)-1 .
$$

Thus we get $w\left(z^{\prime}\right)=1 / m$ and $w\left(u^{\prime}\right)=1$ since $w\left(u^{\prime}\right)$ is a positive integer.

For the standard embedding $j$, we denote $\tau$-wt $(z)=1 / m, \tau$-wt $(u)=1$ and assume that $\tau-w t(f(z, u))=k$. This $k$ is a positive integer. We define $\sigma_{a, b}=\frac{1}{m}(a$, $b, 1, m)$ and $v_{a, b}=\left(j, \sigma_{a, b}\right)$ for positive integers $a$ and $b$.

6.3. Proposition. For the standard embedding $j$, we have

$$
\mathscr{W}_{1 / m}(j)=\left\{v_{a, b} \mid 0<a, b \in \mathbb{Z}, a+b \leq m k, a \equiv-b \equiv \alpha(\bmod m)\right\}
$$

In particular, maximal elements in $\mathscr{W}_{1 / m}(j)$ are those $v_{a, b}$ with $a+b=m k$.

Proof. If $(j, \sigma) \in \mathscr{W}_{1 / m}(j)$, then $\sigma-w t(z)=1 / m$ and $\sigma-w t(u)=1$ by (6.2). It follows from (3.9.1) that $\sigma$-wt $(x y)=\sigma$-wt $(\varphi)$. We also have $\sigma$-wt $(\varphi) \leq \tau$-wt $(f(z$, $u)$ ). Hence $a+b \leq m k$. Since $\sigma \in \mathbb{Z}^{4}+\frac{1}{m}(\alpha,-\alpha, 1,0) \mathbb{Z}$, we get the desired result.

6.4. Theorem. For each $v_{a, b} \in \mathscr{W}_{1 / m}(j)$ with $a+b=m k$, the $v_{a, b}$-blow up $\pi_{a, b}: \bar{X}_{a, b} \rightarrow X$ is divisorial with discrepancy $1 / m$ and $\sum_{Q \in \bar{X}_{a, b}}\left(a w\left(\bar{X}_{a, b}, Q\right)-1\right)=$ $\max \{\operatorname{aw}(X)-k-1,0\}$. These $\pi_{a, b}$ are not mutually isomorphic over $X$. Furthermore, there are exactly divisors with discrepancies $1 / m$ over $X$.

Proof. We first show that $\bar{X}_{a, b}$ has only terminal singularities. Let $E_{a, b}$ be the exceptional divisor of $\pi_{a, b}$. Let $Q_{1}$ (resp. $Q_{2}, Q_{4}$ ) be the origin of the $x$-chart (resp. $y$-chart, $u$-chart). Since

$$
E_{a, b} \simeq\left\{x y+f_{\tau-w t=k}(z, u)=0\right\} \subseteq \mathbb{P}(a, b, 1, m),
$$

we see that $E_{a, b}$ is Cartier outside $\left\{Q_{1}, Q_{2}, Q_{4}\right\} \cap E_{a, b}$ and that

$$
\operatorname{Sing}\left(E_{a, b}\right) \subseteq\left\{Q_{1}, Q_{2}\right\} \cup\{x=y=0\}
$$

By (3.2), $\bar{X}_{a, b}$ is covered by the following four affine open sets : 


$$
\begin{aligned}
& U_{1}=\left\{\bar{y}+f\left(\bar{x}^{1 / m} \bar{z}, \bar{x} \bar{u}\right) / \bar{x}^{k}=0\right\} / \mathbb{Z}_{a}(m,-b,-1,-m), \\
& U_{2}=\left\{\bar{x}+f\left(\bar{y}^{1 / m} \bar{z}, \bar{y} \bar{u}\right) / \bar{y}^{k}=0\right\} / \mathbb{Z}_{b}(-a, m,-1,-m), \\
& U_{3}=\left\{\bar{x} \bar{y}+f\left(\bar{z}^{1 / m}, \bar{z} \bar{u}\right) / \bar{z}^{k}=0\right\} \subseteq \mathbb{C}^{4}, \\
& U_{4}=\left\{\bar{x} \bar{y}+f\left(\bar{z} \bar{u}^{1 / m}, \bar{u}\right) / \bar{u}^{k}=0\right\} \mathbb{Z}_{m}(a, b, 1,0) .
\end{aligned}
$$

Since $X$ has only isolated singularities and $\pi_{a, b}$ is isomorphic over the smooth locus of $X, \bar{X}_{a, b}$ has singularities only on $E_{a, b}$. By the above description, the origin $Q_{1}$ of $U_{1}$ is isomorphic to $(\bar{x}, \bar{z}, \bar{u}) / \mathbb{Z}_{a}(m,-1,-m)$ and the origin $Q_{2}$ of $U_{2}$ is isomorphic to $(\bar{y}, \bar{z}, \bar{u}) / \mathbb{Z}_{b}(m,-1,-m)$. We also see that $\bar{X}_{a, b}$ has only isolated cDV points along the $\bar{u}$-axis of $U_{3}$, and the origin $Q_{4}$ of $U_{4}$ is at worst terminal of type $(\mathrm{cA} / \mathrm{m})$. Thus we see that $\bar{X}_{a, b}$ has only terminal singularities. We also have

$$
\sum_{Q \in \bar{X}_{a, b}}\left(\operatorname{aw}\left(\bar{X}_{a, b}, Q\right)-1\right)= \begin{cases}\operatorname{aw}\left(\bar{X}_{a, b}, Q_{4}\right)-1=\operatorname{aw}(X)-k-1 & \text { if } Q_{4} \in \bar{X}_{a, b}, \\ 0 & \text { if } Q_{4} \notin \bar{X}_{a, b} .\end{cases}
$$

Since $Q_{4} \in X_{a, b}$ if and only if aw $(X)>k$, we see that $\sum_{Q \in \bar{X}_{a, b}}\left(\operatorname{aw}\left(\bar{X}_{a, b}, Q\right)-1\right)=$ $\max \{\operatorname{aw}(X)-k-1,0\}$.

Since $E_{a, b}$ is irreducible, we have $K_{\bar{X}_{a, b}}=\pi_{a, b}^{*}\left(K_{X}\right)+\frac{1}{m} E_{a, b}$. Therefore $\pi_{a, b}$ is divisorial with discrepancy $1 / \mathrm{m}$.

Let $D$ be the $\mathbb{Q}$-Cartier Weil divisor on $X$ defined by $x=0$. By (3.5), we have

$$
\pi_{a, b}^{*}(D)=\pi_{a, b}^{-1}[D]+\frac{a}{m} E_{a, b}
$$

Hence these blow ups are not isomorphic over $X$.

In order to count the number of divisors with discrepancies $1 / m$, we first take some $v_{a, b} \in \mathscr{W}_{1 / m}$ with $a+b=m k$ and blow up as above. By (5.3), singularities on $\bar{X}_{a, b}$ with index $\leq m$ will not produce divisors with discrepancies $1 / m$ over $X$. So we shall study singularities on $\bar{X}_{a, b}$ with index $>m$. We can resolve the origin $Q_{1}$ of $U_{1}$ by using (5.1). There is a projective birational morphism $\nu: Z \rightarrow \bar{X}_{a, b}$ such that

$$
K_{Z}=\nu^{*}\left(K_{\bar{X}_{a, b}}\right)+\sum_{i=1}^{a-1} \frac{i}{a} F_{i},
$$

where $\sum_{i=1}^{a-1} F_{i}$ is the exceptional divisor of $\nu$ over $Q_{1}$. Since $E_{a, b}$ is defined by $\bar{x}=$ 0 near $Q_{1}$, we have

$$
\nu^{*}\left(E_{a, b}\right)=\nu^{-1}\left[E_{a, b}\right]+\sum_{i=1}^{a-1}\left\langle-\frac{m i}{a}\right\rangle F_{i}
$$

By (5.3), we have 


$$
a\left(F_{i}, X\right)=\frac{i}{a}+\frac{1}{m}\left\langle-\frac{m i}{a}\right\rangle \text {. }
$$

Hence $a\left(F_{i}, X\right)=1 / m$ if and only if $i=1,2, \ldots,[a / m]$. Similarly there are exactly $[b / m]$ divisors with discrepancies $1 / m$ over $Q_{2}$. Including $E_{a, b}$, the number of divisors with discrepancies $1 / m$ is $[a / m]+[b / m]+1=k$ since $a$ and $b$ are both prime to $m$.

6.5. Proposition. If $v^{\prime}=\left(j^{\prime}, \sigma^{\prime}\right) \in \mathscr{W}_{1 / m}$ is maximal, then there exists $v_{a, b}$ with $a+b=m k$ such that $v^{\prime}>v_{a, b}$.

Proof. Let $\mathscr{A}=\left\{v_{a, b} \in \mathscr{W}_{1 / m}(j) \mid v_{a, b}<v^{\prime}\right\}$. By (5.4), we have $v^{\prime}>v_{a_{0}, b_{0}}$ if $a_{0}$, $b_{0}<m$. Thus we see that $\mathscr{A} \neq \varnothing$. Hence there is a maximal element $v_{a, b} \in \mathscr{A}$. Assuming that $a+b<m k$, we shall derive a contradiction. Let $\chi:\left(x^{\prime}, y^{\prime}, z^{\prime}, u^{\prime}\right) /$ $\mathbb{Z}_{m} \rightarrow(x, y, z, u) / \mathbb{Z}_{m}$ be a liftable automorphism such that $\chi \circ j^{\prime}=j$. We shall denote

$$
p=\chi^{*}(x), \quad q=\chi^{*}(y), \quad r=\chi^{*}(z), \quad s=\chi^{*}(u) \in \mathbb{C}\left\{x^{\prime}, y^{\prime}, z^{\prime}, u^{\prime}\right\}
$$

Then $\varphi^{\prime}=p q+f(r, s)$ is the defining equation of $j^{\prime}$. Since $v^{\prime}>v_{a, b}$, we have $\sigma^{\prime}-w t(p)$ $\geq a / m$ and $\sigma^{\prime}-w t(q) \geq b / m$. If at least one of these inequalities are strict, then $v^{\prime}>$ $v_{a+m, b}$ or $v^{\prime}>v_{a, b+m}$, which is a contradiction. Hence $\sigma^{\prime}-w t(p)=a / m$ and $\sigma^{\prime}-w t(q)$ $=b / m$. Since $a+b<m k$ and $\sigma^{\prime}-w t(f(r, s)) \geq k$, we have $\sigma^{\prime}-w t\left(\varphi^{\prime}\right)=(a+b) / m$. It follows from (6.2) that, after a permutation of coordinates if necessary,

$$
\sigma^{\prime}-w t s\left(x^{\prime}, y^{\prime}, z^{\prime}, u^{\prime}\right)=\frac{1}{m}\left(a^{\prime}, b^{\prime}, 1, m\right)
$$

for some positive integers $a^{\prime}$ and $b^{\prime}$. Since $d\left(v^{\prime}\right)=1 / m$, we see that $a^{\prime}+b^{\prime}=a+b$. Since $\chi$ is induced by an automorphism of $\mathbb{C}^{4}$, after a permutation of $x^{\prime}$ and $y^{\prime}$ if necessary, we see that $a=a^{\prime}$ and $b=b^{\prime}$. Thus we have $v^{\prime} \sim v_{a, b}$ by (5.5). This also contradict the maximality of $v^{\prime}$. Therefore $a+b=m k$, which completes the proof.

6.6. By (6.4), (6.5) and (5.8), we see that $v_{a, b} \in \mathscr{W}_{1 / m}$ with $a+b=m k$ represent all the maximal elements in $\mathscr{W}_{1 / m} / \sim$. Hence there are exactly $k$ maximal elements in $\mathscr{W}_{1 / m} / \sim$. On the other hand, we have $k$ divisors with discrepancies $1 / m$ over $X$ by (6.4). By (4.2), this completes the proofs for (4.1) and (4.4). (4.5) and (4.6) are the direct consequences of (6.4).

Figure 1 shows the elements of $\mathscr{W}_{1 / m} / \sim$ and their relations, in which we are assuming that $0<\alpha<m$ and $\beta=m-\alpha$. For the $v_{a, b}$-blow up $\pi_{a, b}: \bar{X}_{a, b} \rightarrow X$ with $a$ $+b<m k$, the exceptional divisor of $\pi_{a, b}$ is not irreducible. 


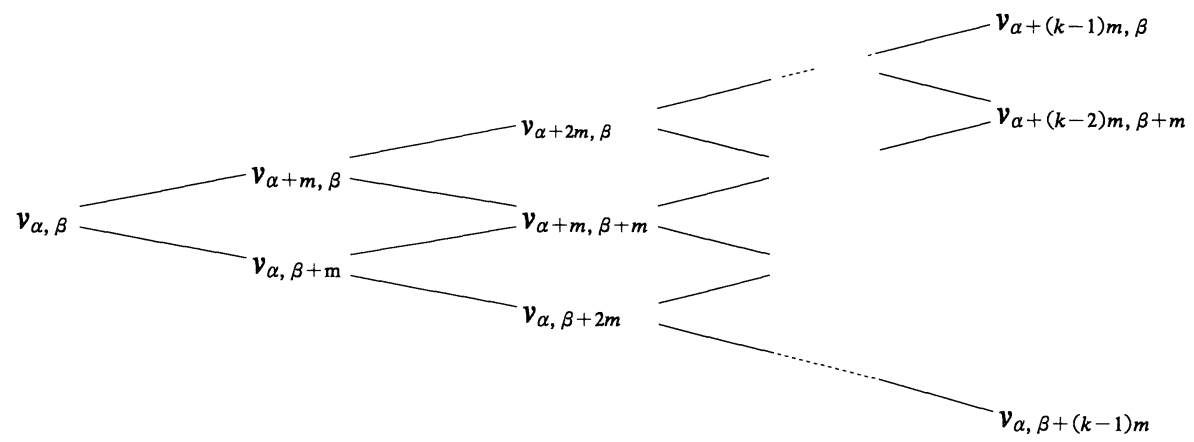

Figure 1. $\mathscr{W}_{1 / m} / \sim$ for $(\mathrm{cA} / \mathrm{m})$ type terminal singularities

\section{§ 7. Terminal Singularities of Type (cAx/4)}

7.1. Let $X$ be a germ of a 3-dimensional terminal singularity of type (cAx/4). By (2.3), there is a standard embedding

$$
j: X \simeq\left\{x^{2}+y^{2}+f(z, u)=0\right\} / \mathbb{Z}_{4}(1,3,1,2) \subseteq(x, y, z, u) / \mathbb{Z}_{4}(1,3,1,2),
$$

where $f(z, u)$ is a $\mathbb{Z}_{4}$-semi-invariant and $u \not f f(z, u)$. In this case, we have aw $(X)$ $=(\operatorname{ord}(f(0, u))+1) / 2$. We denote the defining equation of $j$ as $\varphi=x^{2}+y^{2}+$ $f(z, u)$.

7.2. Lemma. Let $j^{\prime}: X \hookrightarrow\left(x^{\prime}, y^{\prime}, z^{\prime}, u^{\prime}\right) / \mathbb{Z}_{4}(1,3,1,2)$ be an arbitrary embedding, and let $\varphi^{\prime}$ be the defining equation of $j^{\prime}$. Then, after a permutation of coordinates $x^{\prime}$ and $z^{\prime}$ if necessary, we have $x^{\prime 2}, y^{\prime 2} \in \varphi^{\prime}$ or $x^{\prime} z^{\prime}, y^{\prime 2} \in \varphi^{\prime}$. Furthermore, if we assume that $\left(j^{\prime}, \sigma^{\prime}\right) \in \mathscr{W}_{1 / 4}$, then the following holds :

(1) If $x^{\prime 2}, y^{\prime 2} \in \varphi^{\prime}$, then $\sigma^{\prime}=\frac{1}{4}(2 l+1,2 l+3,1,2)$ for some even integer $l$ or $\sigma^{\prime}=$ $\frac{1}{4}(2 l+3,2 l+1,1,2)$ for some odd integer $l$.

(2) If $x^{\prime} z^{\prime}, y^{\prime 2} \in \varphi^{\prime}$, then $\sigma^{\prime}=\frac{1}{4}(1,3,1,2), \frac{1}{4}(1,3,5,2)$ or $\frac{1}{4}(5,3,1,2)$.

Proof. The first part is obvious. For the second part, we write $w=\sigma^{\prime}-w t$. In case (1), since $x^{\prime 2}, y^{\prime 2} \in \varphi^{\prime}$, we have $2 w\left(x^{\prime}\right), 2 w\left(y^{\prime}\right) \geq w\left(\varphi^{\prime}\right)$, in particular $w\left(x^{\prime}\right)+$ $w\left(y^{\prime}\right) \geq w\left(\varphi^{\prime}\right)$. Since $d\left(v^{\prime}\right)=1 / 4$, we have

$$
1 / 4=w\left(x^{\prime}\right)+w\left(y^{\prime}\right)+w\left(z^{\prime}\right)+w\left(u^{\prime}\right)-w\left(\varphi^{\prime}\right)-1 \geq w\left(z^{\prime}\right)+w\left(u^{\prime}\right)-1 .
$$

Since $\sigma^{\prime} \in \mathbb{Z}^{4}+\frac{1}{4}(1,3,1,2) \mathbb{Z}$, we see that $w\left(x^{\prime}\right) \equiv w\left(z^{\prime}\right) \equiv 1 / 4, w\left(y^{\prime}\right) \equiv 3 / 4, w\left(u^{\prime}\right)$ $\equiv w\left(\varphi^{\prime}\right) \equiv 1 / 2 \bmod \mathbb{Z}$. Hence we get $w\left(z^{\prime}\right)=1 / 4$ and $w\left(u^{\prime}\right)=1 / 2$. Then $w\left(x^{\prime}\right)+$ $w\left(y^{\prime}\right)=w\left(\varphi^{\prime}\right)+1 / 2$, so we get $\left|w\left(x^{\prime}\right)-w\left(y^{\prime}\right)\right|=1 / 2$. 
In case (2), we can prove more easily.

For the standard embedding $j$, we denote $\tau$-wt $(z)=1 / 4, \tau-w t(u)=1 / 2$ and assume that $\tau-w t(f(z, u))=(2 k+1) / 2$. Since $\tau-w t(\varphi) \equiv 1 / 2 \bmod \mathbb{Z}$, we see that $k$ is a non-negative integer. For non-negative integers $l$, we define

$$
\sigma_{l}= \begin{cases}\frac{1}{4}(2 l+1,2 l+3,1,2) & \text { if } l \text { is even, } \\ \frac{1}{4}(2 l+3,2 l+1,1,2) & \text { if } l \text { is odd }\end{cases}
$$

and $v_{l}=\left(j, \sigma_{l}\right)$

7.3. Proposition. For the standard embedding $j$, we have

$$
\mathscr{W}_{1 / 4}(j)=\left\{v_{l} \mid l=0,1,2, \ldots, k\right\}
$$

In particular, $v_{k}$ is the unique maximal element in $\mathscr{W}_{1 / 4}(j)$.

Proof. If $\left(j, \sigma_{l}\right) \in \mathscr{W}_{1 / 4}(j)$, then it follows from (3.9.1) that $(2 l+1) / 2=$ $\sigma_{l}-w t(\varphi) \leq \tau-w t(f(z, u))=(2 k+1) / 2$, hence $l \leq k$.

7.4. Theorem. Let $\pi: \bar{X} \rightarrow X$ be the $v_{k}$-blow up and let $E$ be the exceptional divisor of $\pi$. Then $\bar{X}$ has only terminal singularities and $\Sigma_{Q \in \bar{X}}(\operatorname{aw}(\bar{X}, Q)-1)=\max$ $\{\operatorname{aw}(X)-k-2,0\}$. Moreover, if $f_{\tau-w t=(2 k+1) / 2}(z, u)$ is not a square, then $\pi$ is divisorial with discrepancy $1 / 4$ and $E$ is the unique divisor with discrepancy $1 / 4$ over $X$.

Proof. We first show that $\bar{X}$ has only terminal singularities. We shall assume that $k$ is even. Let $Q_{1}$ (resp. $Q_{2}, Q_{4}$ ) be the origin of the $x$-chart (resp. $y$-chart, $u$ chart). Since

$$
E \simeq\left\{x^{2}+f_{\tau-w t=(2 k+1) / 2}(z, u)=0\right\} \subseteq \mathbb{P}(2 k+1,2 k+3,1,2),
$$

we see that $E$ is Cartier outside $\left\{Q_{2}, Q_{4}\right\} \cap E$ and that $\operatorname{Sing}(E) \subseteq\{x=0\}$. By (3.2), $\bar{X}$ is covered by four affine open sets $U_{1}, U_{2}, U_{3}$ and $U_{4}$. Since $Q_{1} \not \subset E$, we do not need $U_{1}$ to cover $\bar{X}$ :

$$
\begin{aligned}
& U_{2}=\left\{\bar{x}^{2}+\bar{y}+f\left(\bar{y}^{1 / 4} \bar{z}, \bar{y}^{1 / 2} \bar{u}\right) / \bar{y}^{(2 k+1) / 2}=0\right\} / \mathbb{Z}_{2 k+3}(2,4,-1,-2), \\
& U_{3}=\left\{\bar{x}^{2}+\bar{z} \bar{y}^{2}+f\left(\bar{z}^{1 / 4}, \bar{z}^{1 / 2} \bar{u}\right) / \bar{z}^{(2 k+1) / 2}=0\right\} \subseteq \mathbb{C}^{4}, \\
& U_{4}=\left\{\bar{x}^{2}+\bar{u} \bar{y}^{2}+f\left(\bar{u}^{1 / 4} \bar{z}, \bar{u}^{1 / 2}\right) / \bar{u}^{(2 k+1) / 2}=0\right\} / \mathbb{Z}_{2}(1,1,1,0) .
\end{aligned}
$$


The origin $Q_{2}$ of $U_{2}$ is isomorphic to $(\bar{x}, \bar{z}, \bar{u}) / \mathbb{Z}_{2 k+3}(2,-1,-2)$, the origin $Q_{4}$ of $U_{4}$ is at worst terminal of type $(\mathrm{cD} / 2)$, and other singularities of $\bar{X}$ are all isolated cDV points. Hence $\bar{X}$ has only terminal singularities. We also have

$$
\sum_{Q \in X}(\operatorname{aw}(\bar{X}, Q)-1)= \begin{cases}\operatorname{aw}\left(\bar{X}, Q_{4}\right)-1=\operatorname{aw}(X)-k-2 & \text { if } Q_{4} \in \bar{X}, \\ 0 & \text { if } Q_{4} \notin \bar{X} .\end{cases}
$$

Since $Q_{4} \in \bar{X}$ if and only if aw $(X)>k+1$, we see that $\Sigma_{Q \in \bar{X}}(\operatorname{aw}(\bar{X}, Q)-1)=\max$ $\{$ aw $(X)-k-2,0\}$.

It is easy to see that $E$ is reducible if and only if $f_{\tau-w t=(2 k+1) / 2}(z, u)$ is a square. Thus we know that if $f_{\tau-w t=(2 k+1) / 2}(z, u)$ is not a square, then $\pi$ is a divisorial blow up with discrepancy $1 / 4$.

In order to count the number of divisors with discrepancies $1 / 4$, we shall study each singularity on $\bar{X}$. We can resolve the origin $Q_{2}$ of $U_{2}$ by using (5.1) and get a projective birational morphism $\nu: Z \rightarrow \bar{X}$ such that

$$
K_{Z}=\nu^{*}\left(K_{\bar{X}}\right)+\sum_{i=1}^{2 k+2} \frac{i}{2 k+3} F_{i},
$$

where $\sum_{i=1}^{2 k+2} F_{i}$ is the exceptional divisor of $\nu$ over $Q_{2}$. Since $E$ is defined by $\bar{x}^{2}+$ $f_{\tau-w t=(2 k+1) / 2}(\bar{z}, \bar{u})$ near $Q_{2}$, we have

$$
\nu^{*}(E)=\nu^{-1}[E]+\sum_{i=1}^{k+1} \frac{4 k+6-4 i}{2 k+3} F_{i}+\sum_{i=k+2}^{2 k+2} d_{i} F_{i}
$$

for some $d_{i}>0$. By (5.3), we have

$$
a\left(F_{i}, X\right)= \begin{cases}\frac{i}{2 k+3}+\frac{1}{4} \cdot \frac{4 k+6-4 i}{2 k+3}=\frac{1}{2} & \text { if } i=1, \ldots, k+1, \\ \frac{i}{2 k+3}+\frac{1}{4} d_{i}>\frac{1}{2} & \text { if } i=k+2, \ldots, 2 k+2 .\end{cases}
$$

Thus there are no divisors with discrepancies $1 / 4$ over $Q_{2}$. By (5.3), we see that other singularities on $\bar{X}$ will not produce divisors with discrepancies $1 / 4$ over $X$. Therefore $E$ is the unique divisor with discrepancy $1 / 4$ over $X$.

If $k$ is odd, we can do the same calculation as above by replacing $x$ with $y$.

7.5. Proposition. If $v^{\prime}=\left(j^{\prime}, \sigma^{\prime}\right)$ is maximal in $\mathscr{W}_{1 / 4}$, then $v^{\prime}>v_{k}$.

Proof. Let $l=\max \left\{l \mid v^{\prime}>v_{l}\right\}$. Since $v^{\prime}>v_{0}$, we see that $l \geq 0$. Assuming that $l<k$, we shall derive a contradiction. Let $\chi:\left(x^{\prime}, y^{\prime}, z^{\prime}, u^{\prime}\right) / \mathbb{Z}_{4} \rightarrow(x, y, z, u) / \mathbb{Z}_{4}$ be a liftable automorphism such that $\chi \circ j^{\prime}=j$, then $\varphi^{\prime}=\chi^{*}(\varphi)$ is the defining equation of $j^{\prime}$. We first assume that $l$ is even. Since $v^{\prime}>v_{l}$, we see that 


$$
\sigma^{\prime}-w t s\left(\chi^{*}(x, y, z, u)\right) \geq \frac{1}{4}(2 l+1,2 l+3,1,2) .
$$

If $\sigma^{\prime}-w t\left(\chi^{*}(x)\right)>(2 l+1) / 4$, then $v^{\prime}>v_{l+1}$, which contradicts the maximality of $l$. Hence $\sigma^{\prime}-w t\left(\chi^{*}(x)\right)=(2 l+1) / 4$. We also see that $\sigma^{\prime}-w t\left(\chi^{*}(f(z, u))\right) \geq$ $\tau-w t(f(z, u))=(2 k+1) / 2$. Hence $\sigma^{\prime}-w t\left(\varphi^{\prime}\right)=\sigma^{\prime}-w t\left(\chi^{*}\left(x^{2}+y^{2}+f(z, u)\right)\right)=$ $(2 l+1) / 2$. By (7.2) and (3.9.1), we have $\sigma^{\prime}=\sigma_{l}$. Therefore $v^{\prime} \sim\left(j, \sigma_{l}\right)$ by (5.5). This also contradicts the maximality of $v^{\prime}$ since $l<k$. Similarly, we can derive a contradiction if $l$ is odd.

7.6. In the case $f_{\tau-w t}=(2 k+1) / 2(z, u)$ is not a square, we have $v^{\prime} \sim v_{k}$ for every maximal element $v^{\prime} \in \mathscr{W}_{1 / 4}$ by (7.4), (7.5) and (5.8). Thus (4.1) and (4.4) hold. By (7.4), we also see that (4.5) and (4.6) hold. Figure 2 shows the elements of $\mathscr{W}_{1 / 4} / \sim$ and their relations.

7.7. We shall assume that $f_{\tau-w t=(2 k+1) / 2}(z, u)$ is a square in the rest of this section. Let $f_{\tau-w t=(2 k+1) / 2}(z, u)=-g(z, u)^{2}$. By (7.4), the exceptional divisor of $v_{k}$-blow up is reducible. This naturally leads to the following automorphisms and embeddings. We only treat the case $k$ is even, since otherwise the same proof below will work by replacing $x$ with $y$. Let $\chi_{ \pm}:(x, y, z, u) / \mathbb{Z}_{4} \rightarrow\left(x_{1}, y_{1}, z_{1}, u_{1}\right) / \mathbb{Z}_{4}$ be the automorphisms defined by

$$
\chi_{ \pm}^{*}\left(x_{1}\right)=x \pm g(z, u), \quad \chi_{ \pm}^{*}\left(y_{1}\right)=y, \quad \chi_{ \pm}^{*}\left(z_{1}\right)=z \quad \text { and } \quad \chi_{ \pm}^{*}\left(u_{1}\right)=u,
$$

and let $j_{ \pm}=\chi_{ \pm} \circ j: X \hookrightarrow\left(x_{1}, y_{1}, z_{1}, u_{1}\right) / \mathbb{Z}_{4}(1,3,1,2)$ be the embeddings. Then the defining equations of $j_{ \pm}$are

$$
\varphi_{ \pm}=x_{1}^{2} \mp 2 x_{1} g\left(z_{1}, u_{1}\right)+y_{1}^{2}+h\left(z_{1}, u_{1}\right)
$$

where $h(z, u)=f_{\tau-w t>(2 k+1) / 2}(z, u)$.

7.8. Proposition. For each embedding $j_{ \pm}$, we have

$$
\mathscr{W}_{1 / 4}\left(j_{ \pm}\right)=\left\{\left(j_{ \pm}, \sigma_{l}\right) \mid l=0,1,2, \ldots, k+1\right\}
$$

In particular, $v_{ \pm}=\left(j_{ \pm}, \sigma_{k+1}\right)$ is the unique maximal element in $\mathscr{W}_{1 / 4}\left(j_{ \pm}\right)$.

Proof. Assume that $\left(j_{ \pm}, \sigma_{l}\right) \in \mathscr{W}_{1 / 4}$. Then one sees that $\sigma_{l}-w t\left(\varphi_{ \pm}\right)=(2 l+$ 1) $/ 2$. On the other hand, $\sigma_{l}-w t\left(\varphi_{ \pm}\right) \leq \sigma_{l}-w t\left(x_{1} g\left(z_{1}, u_{1}\right)\right) \leq(k+l+2) / 2$, hence we have $l \leq k+1$.

7.9. Theorem. Let $\pi_{ \pm}: \bar{X}_{ \pm} \rightarrow X$ be the $v_{ \pm}-$blow ups. Then $\pi_{ \pm}$are both divisor- 
ial with discrepancies $1 / 4$ and $\sum_{Q \in \bar{X}_{ \pm}}\left(\operatorname{aw}\left(\bar{X}_{ \pm}, Q\right)-1\right)=\max \{\operatorname{aw}(X)-k-3,0\}$. These $\pi_{+}$and $\pi_{-}$are not isomorphic over $X$. Furthermore, there are exactly 2 divisors with discrepancies $1 / 4$ over $X$.

Proof. Let $E_{ \pm}$be the exceptional divisor of $\pi_{ \pm}$and let $Q_{1}\left(\right.$ resp. $Q_{2}, Q_{4}$ ) be the origin of the $x$-chart (resp. $y$-chart, $u$-chart). Since

$$
E_{ \pm} \simeq\left\{\mp 2 g(z, u) x+y^{2}+h_{\tau-w t=(2 k+3) / 2}(z, u)=0\right\} \subseteq \mathbb{P}(2 k+5,2 k+3,1,2),
$$

we see that $E_{ \pm}$is Cartier outside $\left\{Q_{1}, Q_{4}\right\} \cap E$ and that $\operatorname{Sing}(E) \subseteq\{y=0\}$. As in the proof of (7.4), $\bar{X}_{ \pm}$is covered by three affine open sets :

$$
\begin{aligned}
& U_{1}=\left\{\bar{x} \mp 2 g(\bar{z}, \bar{u})+\bar{y}^{2}+h\left(\bar{x}^{1 / 4} \bar{z}, \bar{x}^{1 / 2} \bar{u}\right) / \bar{x}^{(2 k+3) / 2}=0\right\} / \mathbb{Z}_{2 k+5}(4,2,-1,-2), \\
& U_{3}=\left\{\bar{x}^{2} \bar{z} \mp 2 g(1, \bar{u}) \bar{x}+\bar{y}^{2}+h\left(\bar{z}^{1 / 4}, \bar{z}^{1 / 2} \bar{u}\right) / \bar{z}^{(2 k+3) / 2}=0\right\} \subseteq \mathbb{C}^{4}, \\
& U_{4}=\left\{\bar{x}^{2} \bar{u} \mp 2 g(\bar{z}, 1) \bar{x}+\bar{y}^{2}+h\left(\bar{u}^{1 / 4} \bar{z}, \bar{u}^{1 / 2}\right) / \bar{u}^{(2 k+3) / 2}=0\right\} / \mathbb{Z}_{2}(1,1,1,0) .
\end{aligned}
$$

The origin $Q_{1}$ of $U_{1}$ is isomorphic to $(\bar{y}, \bar{z}, \bar{u}) / \mathbb{Z}_{2 k+5}(2,-1,-2)$, the origin $Q_{4}$ of $U_{4}$ is at worst terminal of type $(\mathrm{cD} / 2)$, and other singularities on $\bar{X}$ are all isolated cDV points. Hence $\bar{X}_{ \pm}$has only terminal singularities. We also have

$$
\sum_{Q \in \bar{X}_{ \pm}}\left(\operatorname{aw}\left(\bar{X}_{ \pm}, Q\right)-1\right)= \begin{cases}\operatorname{aw}\left(\bar{X}_{ \pm}, Q_{4}\right)-1=\operatorname{aw}(X)-k-3 & \text { if } Q_{4} \in \bar{X}_{ \pm}, \\ 0 & \text { if } Q_{4} \not \subset \bar{X}_{ \pm} .\end{cases}
$$

Since $Q_{4} \in \bar{X}_{ \pm}$if and only if aw $(X)>k+2$, we see that $\sum_{Q \in \bar{X}_{ \pm}}\left(\operatorname{aw}\left(\bar{X}_{ \pm}, Q\right)-1\right)=$ $\max \{\operatorname{aw}(X)-k-3,0\}$.

Since $E_{ \pm}$is an irreducible divisor, we have $K_{\bar{X}_{ \pm}}=\pi_{ \pm}^{*}\left(K_{X}\right)+\frac{1}{4} E_{ \pm}$. Thus $\pi_{ \pm}$are both divisorial with discrepancies $1 / 4$.

Let $D$ be the $\mathbb{Q}$-Cartier Weil divisor on $X$ defined by $x+g(z, u)=0$. By (3.5), we have

$$
\pi_{+}^{*}(D)=\pi_{+}^{-1}[D]+\frac{2 k+5}{4} E_{+} \quad \text { and } \quad \pi_{-}^{*}(D)=\pi_{-}^{-1}[D]+\frac{2 k+1}{4} E_{-} .
$$

Hence $\pi_{+}$and $\pi_{-}$are not isomorphic over $X$.

In order to count the number of divisors with discrepancies $1 / 4$, we shall use $\pi_{+}$as the first blow up. We can resolve the origin $Q_{1}$ of $U_{1}$ by (5.1) and get a projective birational morphism $\nu: Z \rightarrow \bar{X}_{+}$such that

$$
K_{Z}=\nu^{*}\left(K_{\bar{X}_{+}}\right)+\sum_{i=1}^{2 k+4} \frac{i}{2 k+5} F_{i}
$$

where $\sum_{i=1}^{2 k+4} F_{i}$ is the exceptional divisor of $\nu$ over $Q_{1}$. Since $E_{+}$is defined by $-2 g(\bar{z}, \bar{u})+\bar{y}^{2}+h_{\tau-w t=(2 k+3) / 2}(\bar{z}, \bar{u})=0$ near $Q_{1}$, we have 


$$
\nu^{*}\left(E_{+}\right)=\nu^{-1}\left[E_{+}\right]+\frac{2 k+1}{2 k+5} F_{1}+\sum_{i=2}^{k+2} \frac{4 k+10-4 i}{2 k+5} F_{i}+\sum_{i=k+3}^{2 k+4} d_{i} F_{i}
$$

where $d_{i}>0$. Hence we get

$$
a\left(F_{i}, X\right)= \begin{cases}\frac{1}{2 k+5}+\frac{1}{4} \cdot \frac{2 k+1}{2 k+5}=\frac{1}{4} & \text { if } i=1, \\ \frac{i}{2 k+5}+\frac{1}{4} \cdot \frac{4 k+10-4 i}{2 k+5}=\frac{1}{2} & \text { if } i=2, \ldots, k+2, \\ \frac{i}{2 k+5}+\frac{1}{4} d_{i}>\frac{1}{2} & \text { if } i=k+3, \ldots, 2 k+4 .\end{cases}
$$

By (5.3), we see that other singularities on $\bar{X}_{+}$will not produce divisors with discrepancies $1 / 4$. Therefore $E_{+}$and $F_{1}$ are the divisors with discrepancies $1 / 4$ over $X$.

7.10. Proposition. If $v^{\prime}=\left(j^{\prime}, \sigma^{\prime}\right)$ is a maximal element in $\mathscr{W}_{1 / 4}$, then $v^{\prime}>v_{+}$ or $v^{\prime}>v_{-}$.

Proof. We shall show that $v^{\prime}>v_{-}$assuming $v^{\prime} \ngtr v_{+}$. Let $\chi:\left(x^{\prime}, y^{\prime}, z^{\prime}, u^{\prime}\right) / \mathbb{Z}_{4}$ $\rightarrow\left(x_{1}, y_{1}, z_{1}, u_{1}\right) / \mathbb{Z}_{4}$ be a liftable automorphism such that $\chi \circ j^{\prime}=j_{+}$. Then $\varphi^{\prime}=$ $\chi^{*}\left(\varphi_{+}\right)$is a defining equation of $j^{\prime}$. By (7.5), we see that $v^{\prime}>v_{k} \sim\left(j_{+}, \sigma_{k}\right)$. Hence we get

$$
\sigma^{\prime}-w t s\left(\chi^{*}\left(x_{1}, y_{1}, z_{1}, u_{1}\right)\right) \geq \frac{1}{4}(2 k+1,2 k+3,1,2) .
$$

Our assumption $v^{\prime} \ngtr v_{+}$implies $\sigma^{\prime}-w t\left(\chi^{*}\left(x_{1}\right)\right)=(2 k+1) / 4$. If we assume that $\sigma^{\prime}-w t\left(\chi^{*}\left(x_{1}-2 g\left(Z_{1}, u_{1}\right)\right)\right)=(2 k+1) / 4$, then $\sigma^{\prime}-w t\left(\varphi^{\prime}\right)=\sigma^{\prime}-w t\left(\chi^{*}(x(x-2 g(z\right.$, $\left.\left.u))+y^{2}+h(z, u)\right)\right)=(2 k+1) / 2$. Since $d\left(v^{\prime}\right)=1 / 4$, (3.9.1) and (7.2) imply that $\sigma^{\prime}=\sigma_{k}$. By (5.5), we see that $v^{\prime} \sim\left(j_{+}, \sigma_{k}\right)$ is not maximal. Therefore $\sigma^{\prime}-w t\left(\chi^{*}\left(x_{1}\right.\right.$ $\left.\left.-2 g\left(z_{1}, u_{1}\right)\right)\right) \geq(2 k+5) / 4$, and this shows that $v^{\prime}>v_{-}$.

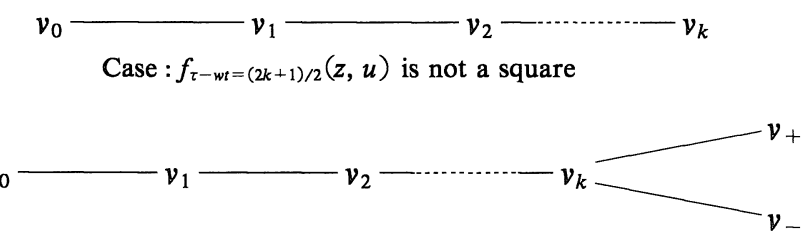

Case $: f_{\tau-w t}=(2 k+1) / 2(z, u)$ is a square

Figure 2. $\mathscr{W}_{1 / 4}$ for $(\mathrm{cAx} / 4)$ type terminal singularities 
7.11. In the case $f_{\tau-w t=(2 k+1) / 2}(z, u)$ is a square, we have $v^{\prime} \sim v_{+}$or $v^{\prime} \sim v_{-}$for every maximal element $v^{\prime} \in \mathscr{W}_{1 / 4}$ by (7.9), (7.10) and (5.8). Thus (4.1) and (4.4) hold. By (7.9), we also see that (4.5) and (4.6) hold. Figure 2 shows the elements of $\mathscr{W}_{1 / 4} / \sim$ and their relations.

\section{§ 8. Terminal Singularities of Type (cAx/2)}

8.1. Let $X$ be a germ of a 3-dimensional terminal singularity of type (cAx/2). By (2.3), there is a standard embedding

$$
j: X \simeq\left\{x^{2}+y^{2}+f(z, u)=0\right\} / \mathbb{Z}_{2}(0,1,1,1) \subseteq(x, y, z, u) / \mathbb{Z}_{2}(0,1,1,1)
$$

where $f(z, u) \in(z, u)^{4} \mathbb{C}\{z, u\}$ is a $\mathbb{Z}_{2}$-invariant. In this case, we have aw $(X)=2$.

The discussions below are almost the same as the one in section 7 and the proofs can be done by a similar method. So we shall omit the proofs here.

8.2. Lemma. Let $j^{\prime}: X \hookrightarrow\left(x^{\prime}, y^{\prime}, z^{\prime}, u^{\prime}\right) / \mathbb{Z}_{2}(0,1,1,1)$ be an arbitrary embedding, and let $\varphi^{\prime}$ be the defining equation of $j^{\prime}$. Then, after a permutation of coordinates if necessary, we have $x^{\prime 2}, y^{\prime 2} \in \varphi^{\prime}$. Furthermore, assume that $\left(j^{\prime}, \sigma^{\prime}\right) \in$ $\mathscr{W}_{1 / 2}$, then $\sigma^{\prime}=\frac{1}{2}(l, l+1,1,1)$ for some even integer $l$ or $\sigma^{\prime}=\frac{1}{2}(l+1, l, 1,1)$ for some odd integer $l$.

For the embedding $j$, we denote $\tau$-wt $(z)=\tau$-wt $(u)=1 / 2$, and assume that $\tau$-wt $(f(z, u))=k$. Then $k$ is a positive integer. For positive integers $l$, we define

$$
\sigma_{l}= \begin{cases}\frac{1}{2}(l, l+1,1,1), & \text { if } l \text { is even, } \\ \frac{1}{2}(l+1, l, 1,1), & \text { if } l \text { is odd },\end{cases}
$$

and $v_{l}=\left(j, \sigma_{l}\right)$.

8.3. Proposition. For the standard embedding $j$, we have

$$
\mathscr{W}_{1 / 2}(j)=\left\{v_{l} \mid l=1,2, \ldots, k\right\}
$$

In particular, $v_{k}$ is the unique maximal element in $\mathscr{W}_{1 / 2}(j)$.

8.4. Theorem. Let $\pi: \bar{X} \rightarrow X$ be the $v_{k}$-blow up and let $E$ be the exceptional 
divisor of $\pi$. Then $\bar{X}$ has only terminal singularties and $\Sigma_{Q \in \bar{X}}(\operatorname{aw}(\bar{X}, Q)-1)=0$. Moreover, if $f_{\tau-w t=k}(z, u)$ is not a square, then $\pi$ is divisorial with discrepancy $1 / 2$ and $E$ is the unique divisor with discrepancy $1 / 2$ over $X$.

8.5. Proposition. If $v^{\prime}=\left(j^{\prime}, \sigma^{\prime}\right)$ is a maximal element in $\mathscr{W}_{1 / 2}$, then $v^{\prime}>v_{k}$.

8.6. We shall assume that $f_{\tau-w t=k}(z, u)$ is a square in the rest of this section. Let $f_{\tau-w t=k}(z, u)=-g(z, u)^{2}$. As in section 7, we only treat the case $k$ is even.

Let $\chi_{ \pm}:(x, y, z, u) / \mathbb{Z}_{2} \rightarrow\left(x_{1}, y_{1}, z_{1}, u_{1}\right) / \mathbb{Z}_{2}$ be the automorphisms defined by

$$
\chi_{ \pm}^{*}\left(x_{1}\right)=x \pm g(z, u), \quad \chi_{ \pm}^{*}\left(y_{1}\right)=y, \quad \chi_{ \pm}^{*}\left(z_{1}\right)=z \quad \text { and } \quad \chi_{ \pm}\left(u_{1}\right)=u,
$$

and let $j_{ \pm}=\chi_{ \pm} \circ j: X \hookrightarrow\left(x_{1}, y_{1}, z_{1}, u_{1}\right) / \mathbb{Z}_{2}(0,1,1,1)$ be the embeddings. Then the defining equations of $j_{ \pm}$are

$$
\varphi_{ \pm}=x_{1}^{2} \mp 2 x_{1} g\left(z_{1}, u_{1}\right)+y_{1}^{2}+h\left(z_{1}, u_{1}\right)
$$

where $h(z, u)=f_{\tau-w t>k}(z, u)$.

8.7. Proposition. For each embedding $j_{ \pm}$, we have

$$
\mathscr{W}_{1 / 2}\left(j_{ \pm}\right)=\left\{\left(j_{ \pm}, \sigma_{l}\right) \mid l=1,2, \ldots, k+1\right\}
$$

In particular, $v_{ \pm}=\left(j_{ \pm}, \sigma_{k+1}\right)$ is the unique maximal element in $\mathscr{W}_{1 / 2}\left(j_{ \pm}\right)$.

8.8. Theorem. Let $\pi_{ \pm}: \bar{X}_{ \pm} \rightarrow X$ be the $v_{ \pm}$-blow ups. Then $\pi_{ \pm}$are both divisorial with discrepancies $1 / 2$ and $\sum_{Q \in \bar{X}_{+}}(\operatorname{aw}(\bar{X}, Q)-1)=0$. These $\pi_{+}$and $\pi_{-}$are not isomorphic over $X$. Furtheremore, there are exactly 2 divisors with discrepancies $1 / 2$ over $X$.

8.9. Proposition. If $v^{\prime}=\left(j^{\prime}, \sigma^{\prime}\right)$ is a maximal element in $\mathscr{W}_{1 / 2}$, then $v^{\prime}>v_{+}$ or $v^{\prime}>v_{-}$.

8.10. By the same discussion as in section 7 , the above propositions and theorems complete the proofs of (4.1), (4.4), (4.5) and (4.6). The elements of $\mathscr{W}_{1 / 2} / \sim$ and their relations are almost the same as in Figure 2. (The minimal element is $v_{1}$ in this case.)

\section{§ 9. Terminal Singularities of Type (cD/3)}

9.1. Let $X$ be a germ of a 3-dimensional terminal singularity of type (cD/3). 
By (2.3), there is a standard embedding

$$
j: X \simeq\{\varphi(x, y, z, u)=0\} / \mathbb{Z}_{3}(2,1,1,0) \subseteq(x, y, z, u) / \mathbb{Z}_{3}(2,1,1,0) .
$$

In the above expression,

$$
\varphi=\left\{\begin{array}{l}
u^{2}+x^{3}+y z(y+z), \\
u^{2}+x^{3}+y z^{2}+x y^{4} \lambda\left(y^{3}\right)+y^{6} \mu\left(y^{3}\right), \quad \text { or } \\
u^{2}+x^{3}+y^{3}+x y z^{3} \alpha\left(z^{3}\right)+x z^{4} \beta\left(z^{3}\right)+y z^{5} \gamma\left(z^{3}\right)+z^{6} \delta\left(z^{3}\right),
\end{array}\right.
$$

where $\lambda\left(y^{3}\right), \mu\left(y^{3}\right) \in \mathbb{C}\left\{y^{3}\right\}, 4 \lambda^{3}+27 \mu^{2} \neq 0, \alpha\left(z^{3}\right), \beta\left(z^{3}\right), \gamma\left(z^{3}\right), \delta\left(z^{3}\right) \in \mathbb{C}\left\{z^{3}\right\}$. In this case, we have aw $(X)=2$.

As before, we first determine all the possible weights $\sigma^{\prime}$ such that $\left(j^{\prime}, \sigma^{\prime}\right) \in$ $\mathscr{W}_{1 / 3}$ for some embedding $j^{\prime}: X \hookrightarrow\left(x^{\prime}, y^{\prime}, z^{\prime}, u^{\prime}\right) / \mathbb{Z}_{3}(2,1,1,0)$.

9.2. Lemma. Let $j^{\prime}: X \hookrightarrow\left(x^{\prime}, y^{\prime}, z^{\prime}, u^{\prime}\right) / \mathbb{Z}_{3}(2,1,1,0)$ be an arbitrary embedding and let $\varphi^{\prime}$ be the defining equation of $j^{\prime}$. Then $u^{\prime 2}, x^{\prime 3}$ and some cubic terms in $y^{\prime}$ and $z^{\prime}$ appear in $\varphi^{\prime}$. Furthermore, if $\left(j^{\prime}, \sigma^{\prime}\right) \in \mathscr{W}_{1 / 3}$, then the following holds :

(1) If $y^{\prime 3} \in \varphi^{\prime}$, then $\sigma^{\prime}=\frac{1}{3}(2,1,1,3), \frac{1}{3}(2,4,1,3)$ or $\frac{1}{3}(5,4,1,6)$.

(2) If $y^{\prime 2} z^{\prime} \in \varphi^{\prime}$ or $y^{\prime} z^{\prime 2} \in \varphi^{\prime}$, then $\sigma^{\prime}=\frac{1}{3}(2,1,1,3), \frac{1}{3}(2,4,1,3)$ or $\frac{1}{3}(2,1,4$, 3). In particular, if the cubic part in $y^{\prime}$ and $z^{\prime}$ of $\varphi^{\prime}$ does not have a triple factor, then $\sigma^{\prime}=\frac{1}{3}(2,1,1,3), \frac{1}{3}(2,1,4,3)$ or $\frac{1}{3}(2,4,1,3)$.

Proof. The first part is obvious. Let $w=\sigma^{\prime}-w t$ and $v^{\prime}=\left(j^{\prime}, \sigma^{\prime}\right)$. Then in case (1), we have $3 w\left(x^{\prime}\right) \geq w\left(\varphi^{\prime}\right), 3 w\left(y^{\prime}\right) \geq w\left(\varphi^{\prime}\right)$ and $2 w\left(u^{\prime}\right) \geq w\left(\varphi^{\prime}\right)$. Since $d\left(v^{\prime}\right)=$ $1 / 3$, (3.9.1) implies that

$$
1 / 3=w\left(x^{\prime}\right)+w\left(y^{\prime}\right)+w\left(z^{\prime}\right)+w\left(u^{\prime}\right)-w\left(\varphi^{\prime}\right)-1 \geq w\left(z^{\prime}\right)+w\left(\varphi^{\prime}\right) / 6-1 .
$$

Since $\sigma^{\prime} \in \mathbb{Z}^{4}+\frac{1}{3}(2,1,1,0) \mathbb{Z}$ and $w\left(\varphi^{\prime}\right) \in \mathbb{Z}$, we see that $w\left(x^{\prime}\right) \equiv 2 / 3, w\left(y^{\prime}\right) \equiv$ $w\left(z^{\prime}\right) \equiv 1 / 3, w\left(u^{\prime}\right) \equiv 0 \bmod \mathbb{Z}$. So the above inequality shows that $w\left(z^{\prime}\right)=1 / 3$ and $w\left(\varphi^{\prime}\right) \leq 6$. Using these, the rest follows from case by case analysis. For example, if $w\left(\varphi^{\prime}\right)=6$, then we have $w\left(x^{\prime}\right) \geq 8 / 3, w\left(y^{\prime}\right) \geq 7 / 3, w\left(u^{\prime}\right) \geq 3$. Thus we get $w\left(x^{\prime}\right)+w\left(y^{\prime}\right)+w\left(z^{\prime}\right)+w\left(u^{\prime}\right)-w\left(\varphi^{\prime}\right)-1 \geq 4 / 3$, which is a contradiction. Therefore $w\left(\varphi^{\prime}\right)=6$ does not occur. Case (2) can be treated similarly.

We define 


$$
\begin{aligned}
& \sigma_{0}=\frac{1}{3}(2,1,1,3), \quad \sigma_{1,1}=\frac{1}{3}(2,4,1,3), \\
& \sigma_{1,2}=\frac{1}{3}(2,1,4,3) \quad \text { and } \sigma_{2}=\frac{1}{3}(5,4,1,6) .
\end{aligned}
$$

9.3. Let $j^{\prime}: X \hookrightarrow\left(x^{\prime}, y^{\prime}, z^{\prime}, u^{\prime}\right) / \mathbb{Z}_{3}(2,1,1,0)$ be an embedding and let $\varphi^{\prime}$ be the defining equation of $j^{\prime}$. We shall consider the following conditions on $\varphi^{\prime}$. (See (3.4) for the definition of $I^{\sigma_{1}}, 1(4)$ etc.)

(9.3.1) $\varphi^{\prime} \equiv u^{\prime 2}+x^{\prime 3}+y^{\prime} z^{\prime 2} \bmod I^{\sigma_{1}, 1}$ (3).

(9.3.2) $\varphi^{\prime} \equiv\left(u^{\prime 2}+x^{\prime 3}+x^{\prime} z^{\prime 4} \beta_{0}+z^{\prime 6} \delta_{0}\right)+\left(y^{\prime 2} z^{\prime}+x^{\prime} z^{\prime 7} \beta_{1}+z^{\prime 9} \delta_{1}\right) \bmod I^{\sigma_{1,1}}$ (4) for some $\beta_{0}, \beta_{1}, \delta_{0}, \delta_{1} \in \mathbb{C}$.

(9.3.3) $\varphi^{\prime} \equiv\left(u^{\prime 2}+x^{\prime 3}+x^{\prime} z^{\prime 4} \beta_{0}+z^{\prime 6} \delta_{0}\right)+y^{\prime} z^{\prime 3}\left(x^{\prime} \alpha_{0}+z^{\prime 2} \gamma_{0}\right)+z^{\prime 7}\left(x^{\prime} \beta_{1}+z^{\prime 2} \delta_{1}\right)$ $\bmod I^{\sigma_{1}, 1}(4)$ for some $\alpha_{0}, \ldots, \delta_{1} \in \mathbb{C}$, and $y^{\prime 3} \in \varphi^{\prime}$.

(9.3.4) $\varphi^{\prime}=u^{\prime 2}+\lambda x^{\prime 2} z^{\prime 2}+y^{\prime 3}+\alpha x^{\prime} y^{\prime} z^{\prime 3}+\beta x^{\prime} z^{\prime 7}+\gamma y^{\prime} z^{\prime 8}+\delta z^{\prime 12} \bmod I^{\sigma_{2}}$ (5) for some $\alpha, \beta, \gamma, \delta, \lambda \in \mathbb{C}$ and $x^{\prime 3} \in \varphi^{\prime}$.

9.4. Proposition. Let $\pi: \bar{X} \rightarrow X$ be the $\left(j^{\prime}, \sigma_{1,1}\right)$-blow up and $E$ be the exceptional divisor of $\pi$. Then the following holds :

(1) If (9.3.1) or (9.3.2) holds, then $\pi$ is divisorial with discrepancy $1 / 3$.

(2) If (9.3.3) holds, then $\bar{X}$ has only canonical singularities and $E$ is irreducible. Furthermore, if the system of equations

$$
x^{3}+x \beta_{0}+\delta_{0}=0, \quad 3 x^{2}+\beta_{0}=0, \quad x \alpha_{0}+\gamma_{0}=0, \quad x \beta_{1}+\delta_{1}=0
$$

has no solutions in $x$, then $\pi$ is divisorial with discrepancy $1 / 3$.

(3) Assume that (9.3.1), (9.3.2) or (9.3.3) holds. In case (9.3.3), we further assume that $(*)$ has no solutions. Then the origin of the $y^{\prime}$-chart of $\bar{X}$ is the unique non Gorenstein point and it is terminal of type (cAx/4) with axial weight 2.

Proof. First we assume that (9.3.1) holds. Then $E \simeq\left\{u^{2}+x^{3}+y z^{2}=0\right\} \subseteq \mathbb{P}(2$, $4,1,3)$, and it is irreducible. We easily see that $E$ is Cartier outside

$$
(\{z=u=0\} \cup\{x=y=z=0\}) \cap E=\{(0: 1: 0: 0)\}
$$

and that $\operatorname{Sing}(E)=\{(0: 1: 0: 0)\}$. As in $(3.3), \bar{X}$ is covered by four affine chart $U_{1}, \ldots, U_{4}$. Since $X$ has only isolated singularities, we see that $\bar{X}$ is smooth outside the origin of the $y^{\prime}$-chart $U_{2}$. On the other hand, since

$$
U_{2}=\left\{\bar{u}^{2}+\bar{x}^{3}+\bar{z}^{2}+\bar{y} \phi(\bar{x}, \bar{y}, \bar{z}, \bar{u})=0\right\} / \mathbb{Z}_{4}(2,1,1,3)
$$

for some $\phi \in \mathbb{C}\{\bar{x}, \bar{y}, \bar{z}, \bar{u}\}$, we know that the origin of $U_{2}$ is terminal of type (cAx/ 4) with axial weight 2. Since $d\left(v^{\prime}\right)=1 / 3$, we see that $K_{\bar{X}}=\pi^{*}\left(K_{X}\right)+\frac{1}{3} E$. Thus $\pi$ 
is divisorial with discrepancy $1 / 3$. This proves (1) and (3) when $\varphi^{\prime}$ satisfies (9.3.1).

Next, we assume that $\varphi^{\prime}$ satisfies (9.3.3). Then $E \simeq\left\{u^{2}+x^{3}+x z^{4} \beta_{0}+z^{6} \delta_{0}=0\right\}$ $\subseteq \mathbb{P}(2,4,1,3)$, and it is also irreducible. As before $E$ is Cartier outside $\{(0: 1$ : $0: 0)\}$ and

$$
\operatorname{Sing}(E)=\left\{\begin{array}{lr}
\{(0: 1: 0: 0)\} & \text { if } x^{3}+x \beta_{0}+\delta_{0} \text { has no multiple root, } \\
\{(0: 1: 0: 0)\} \cup\{(\xi: *: 1: 0) \mid * \in \mathbb{C}\} & \text { if }(x-\xi)^{2} \mid x^{3}+x \beta_{0}+\delta_{0}
\end{array}\right.
$$

Thus, in order to study singularities of $\bar{X}$, we need only the $y^{\prime}$-chart $U_{2}$ and the $z^{\prime}$ chart $U_{3}$ :

$$
U_{2}=\left\{\varphi_{2}(\bar{x}, \bar{y}, \bar{z}, \bar{u})=0\right\} / \mathbb{Z}_{4}(2,1,1,3) \quad \text { and } \quad U_{3}=\left\{\varphi_{3}(\bar{x}, \bar{y}, \bar{z}, \bar{u})=0\right\} \subseteq \mathbb{C}^{4} \text {, }
$$

where

$$
\begin{aligned}
& \varphi_{2} \equiv \bar{u}^{2}+\bar{x}^{3}+\bar{x} \bar{z}^{4} \beta_{0}+\bar{z}^{6} \delta_{0} \quad \bmod \bar{y} \mathbb{C}\{\bar{x}, \bar{y}, \bar{z}, \bar{u}\}, \\
& \varphi_{3} \equiv \bar{u}^{2}+\bar{x}^{3}+\bar{x} \beta_{0}+\delta_{0}+\bar{y} \bar{z}\left(\bar{x} \alpha_{0}+\gamma_{0}\right)+\bar{z}\left(\bar{x} \beta_{1}+\delta_{1}\right) \quad \bmod \bar{z}^{2} \mathbb{C}(\bar{x}, \bar{y}, \bar{z}, \bar{u}\} .
\end{aligned}
$$

We also have $\bar{y}^{2} \in \varphi_{2}$ and $\bar{y}^{3} \bar{z}^{2} \in \varphi_{3}$. Since $X$ has only isolated singularities, we see that $\operatorname{Sing}\left(U_{2}\right) \subseteq\{\bar{y}=0\}$ and $\operatorname{Sing}\left(U_{3}\right) \subseteq\{\bar{z}=0\}$.

If (*) has no solutions, then we see that $U_{2}$ and $U_{3}$ have only isolated singularities and these are all isolated $\mathrm{CDV}$ points except at the origin of $U_{2}$. The origin of $U_{2}$ is a terminal singularity of type (cAx/4) with axial weight 2 . If (*) has a solution $x=\xi$, then $\bar{X}$ has 1-dimensional singular locus, which are canonical.

In each case, we have $K_{\bar{X}}=\pi^{*}\left(K_{X}\right)+\frac{1}{3} E$ since $d\left(v^{\prime}\right)=1 / 3$. Hence $\pi$ is divisorial with discrepancy $1 / 3$ if $(*)$ has no solutions. This proves (2) and (3) when $\varphi^{\prime}$ satisfies (9.3.3).

The proof for the case (9.3.2) is almost the same as the one for (9.3.3). In this case, $\operatorname{Sing}(\bar{X})$ is always isolated and $\pi$ is always divisorial with discrepancy $1 / 3$.

9.5. Proposition. Assume that $\varphi^{\prime}$ satisfies (9.3.4). Let $\pi: \bar{X} \rightarrow X$ be the $\left(j^{\prime}, \sigma_{2}\right)$-blow up and let $E$ be the exceptional divisor of $\pi$. Then $\pi$ is divisorial with discrepancy $1 / 3$ and $\bar{X}$ is Gorenstein outside two points. One of them is the origin $Q_{1}$ of the $x^{\prime}$-chart of $\bar{X}$ and it is isomorphic to $(\bar{y}, \bar{z}, \bar{u}) / \mathbb{Z}_{5}(1,4,4)$. The other point $Q_{2}$ is isomorphic to $\mathbb{C}^{3} / \mathbb{Z}_{2}(1,1,1)$. Furthermore, $E$ is defined by $\bar{u}^{2}+\lambda \bar{z}^{2}+\bar{y}^{3}+\alpha \bar{y} \bar{z}^{3}$ $+\beta \bar{z}^{7}+\gamma \bar{y} \bar{z}^{8}+\delta \bar{z}^{12}=0$ near $Q_{1}$.

Proof. This can be proved by almost the same method as (9.4). 


\section{\$ 9.A. Terminal singularities of type $(\mathrm{cD} / 3-1)$}

9.6. In this subsection, we assume that $X$ has a standard embedding $j: X \simeq\left\{u^{2}+x^{3}+y z(y+z)=0\right\} / \mathbb{Z}_{3}(2,1,1,0) \subseteq(x, y, z, u) / \mathbb{Z}_{3}(2,1,1,0)$.

The following is immediate from (9.2).

9.7. Proposition. For the standard embedding $j$, we have

$$
\mathscr{W}_{1 / 3}(j)=\left\{(j, \sigma) \mid \sigma=\sigma_{0}, \sigma_{1,1} \text { or } \sigma_{1,2}\right\}
$$

In particular, $v_{1,1}=\left(j, \sigma_{1,1}\right)$ and $v_{1,2}=\left(j, \sigma_{1,2}\right)$ are the maximal elements in $\mathscr{W}_{1 / 3}(j)$.

9.8. We need another pseudo weighted valuation for our study of (cD/3-1) case. This naturally arises if we consider the $\left(j, \sigma_{0}\right)$-blow up of $X$.

Let $\chi_{1}:(x, y, z, u) / \mathbb{Z}_{3} \rightarrow\left(x_{1}, y_{1}, z_{1}, u_{1}\right) / \mathbb{Z}_{3}$ be the automorphism defined by

$$
\chi_{1}^{*}\left(x_{1}\right)=x, \quad \chi_{1}^{*}\left(y_{1}\right)=y+z, \quad \chi_{1}^{*}\left(z_{1}\right)=z \quad \text { and } \quad \chi_{1}^{*}\left(u_{1}\right)=u \text {. }
$$

We get the embedding $j_{1}=\chi_{1} \circ j: X \hookrightarrow\left(x_{1}, y_{1}, z_{1}, u_{1}\right) / \mathbb{Z}_{3}(2,1,1,0)$. The defining equation of $j_{1}$ is

$$
\varphi_{1}=u_{1}^{2}+x_{1}^{3}+y_{1} z_{1}\left(y_{1}-z_{1}\right)
$$

We denote $v_{1,3}=\left(j_{1}, \sigma_{1,1}\right)$, which is maximal in $\mathscr{W}_{1 / 3}\left(j_{1}\right)$.

9.9. Theorem. For each $i=1,2$ and 3 , the $v_{1, l}$-blow up $\pi_{l}: \bar{X}_{i} \rightarrow X$ is divisorial with discrepancy $1 / 3$ and $\sum_{Q \in \bar{X}_{i}}\left(\operatorname{aw}\left(\bar{X}_{i}, Q\right)-1\right)=1$. These $\pi_{i}$ are not mutually isomorphic over $X$. Furthermore, there are exactly 3 divisors with discrepancies $1 / 3$ over $X$.

Proof. By (9.4) (1), $\pi_{1}$ is divisorial with discrepancy $1 / 3$. We also see from (9.4) (3) that $\sum_{Q \in \bar{X}_{i}}\left(\operatorname{aw}\left(\bar{X}_{i}, Q\right)-1\right)=1$. Similarly, $\pi_{2}$ and $\pi_{3}$ have the same properties.

Let $D$ be the $\mathbb{Q}$-Cartier Weil divisor on $X$ defined by $y=0$ and let $E_{i}$ be the exceptional divisor of $\pi_{i}(i=1,2,3)$. By (3.5), we have

$$
\pi_{1}^{*}(D)=\pi_{1}^{-1}[D]+\frac{4}{3} E_{1} \text { and } \pi_{2}^{*}(D)=\pi_{2}^{-1}[D]+\frac{1}{3} E_{2} .
$$

Hence $\bar{X}_{1}$ and $\bar{X}_{2}$ are not isomorphic over $X$. Similarly $\pi_{1}, \pi_{2}$ and $\pi_{3}$ are not 
isomorphic over $X$.

In order to count the number of divisors with discrepancies $1 / 3$, we use $\pi_{1}$ as the first blow up. By (9.4) (3), $\bar{X}_{1}$ has a unique non Gorenstein point which is of type (cAx/4). By (7.4) and (7.9), every terminal singularity of type (cAx/4) has at most 2 divisors with discrepancies 1/4. Hence there are at most 3 divisors (including $E_{1}$ ) with discrepancies $1 / 3$ over $X$ by (5.3). On the other hand, we already know that there are 3 divisors with discrepancies $1 / 3$ over $X$. Therefore there are exactly 3 divisors with discrepancies $1 / 3$ over $X$.

9.10. Proposition. If $v^{\prime}=\left(j^{\prime}, \sigma^{\prime}\right)$ is a maximal element in $\mathscr{W}_{1 / 3}$, then $v^{\prime}>v_{1, i}$ for some $i=1,2$ or 3 .

Proof. If $\sigma^{\prime}=\sigma_{0}$, then $\boldsymbol{v}^{\prime} \sim\left(j, \sigma_{0}\right)$ is not maximal by (5.4). Hence we may assume that $\sigma^{\prime}=\sigma_{1,1}$ or $\sigma_{1,2}$ by (9.2). By symmetry, we assume that $\sigma^{\prime}=\sigma_{1,1}$. We shall show that $v^{\prime}>v_{1,3}$ assuming that $v^{\prime} \ngtr v_{1,1}$ and $v^{\prime} \ngtr v_{1,2}$. Let $\chi:\left(x^{\prime}, y^{\prime}, z^{\prime}, u^{\prime}\right) /$ $\mathbb{Z}_{3} \rightarrow(x, y, z, u) / \mathbb{Z}_{3}$ be a liftable automorphism such that $\chi \circ j^{\prime}=j$. By our assumption,

$$
\sigma^{\prime}-w t s\left(\chi^{*}(x, y, z, u)\right) \nsupseteq \frac{1}{3}(2,4,1,3) \text { and } ¥ \frac{1}{3}(2,1,4,3) \text {. }
$$

Thus we see that $\sigma^{\prime}-w t\left(\chi^{*}(y)\right)=\sigma^{\prime}-w t\left(\chi^{*}(z)\right)=1 / 3$. For the defining equation $\varphi^{\prime}=\chi^{*}(\varphi)$ of $j^{\prime}$, we have $\sigma^{\prime}-w t\left(\varphi^{\prime}\right)=2$ since $d\left(v^{\prime}\right)=1 / 3$. On the other hand, if $\sigma^{\prime}-w t\left(\chi^{*}(y+z)\right)=1 / 3$, then $\sigma^{\prime}-w t\left(\varphi^{\prime}\right)=\sigma^{\prime}-w t\left(\chi^{*}\left(u^{2}+x^{3}+y z(y+z)\right)\right)=1$, which is a contradiction. Hence $\sigma^{\prime}-w t\left(\chi^{*}(y+z)\right) \geq 4 / 3$, which shows that $v^{\prime}>$ $v_{1,3}$.

9.11. By (9.9) and (9.10), we see that (4.1) and (4.4) hold. (4.5) and (4.6) follows from (9.9). Figure 3(9.A) shows the elements of $\mathscr{W}_{1 / 3} / \sim$ and their relations.

\section{§ 9.B. Terminal singularities of type (cD/3-2)}

9.12. In this subsection, we assume that $X$ has a standard embedding

$$
\begin{aligned}
j: X & \simeq\left\{u^{2}+x^{3}+y z^{2}+x y^{4} \lambda\left(y^{3}\right)+y^{6} \mu\left(y^{3}\right)=0\right\} / \mathbb{Z}_{3}(2,1,1,0) \\
& \subseteq(x, y, z, u) / \mathbb{Z}_{3}(2,1,1,0),
\end{aligned}
$$

where $\lambda\left(y^{3}\right), \mu\left(y^{3}\right) \in \mathbb{C}\left\{y^{3}\right\}$ and $4 \lambda^{3}+27 \mu^{2} \neq 0$.

As before, we first determine $\mathscr{W}_{1 / 3}(j)$. 
9.13. Proposition. For the standard embedding $j$, we have

$$
\mathscr{W}_{1 / 3}(j)=\left\{(j, \sigma) \mid \sigma=\sigma_{0}, \sigma_{1,1} \text { or } \sigma_{1,2}\right\}
$$

In particular, $v_{1,1}=\left(j, \sigma_{1,1}\right)$ and $v_{1,2}=\left(j, \sigma_{1,2}\right)$ are the maximal elements in $\mathscr{W}_{1 / 3}(j)$.

9.14. Theorem. For each $i=1$ and 2, the $v_{1, i}$-blow up $\pi_{i}: \bar{X}_{i} \rightarrow X$ is divisorial with discrepancy $1 / 3$ and $\sum_{Q \in \bar{X}_{i}}\left(\operatorname{at}\left(\bar{X}_{i}, Q\right)-1\right)=1$. These $\pi_{1}$ and $\pi_{2}$ are not isomorphic over $X$. Furthermore, there are exactly 2 divisors with discrepancies $1 / 3$ over $X$.

Proof. By (9.4) (1), $\pi_{1}$ is divisorial with discrepancy 1/3. By changing $y$ and $z$ and applying $(9.4)(1), \pi_{2}$ is also divisorial with discrepancy $1 / 3$. Thus we get 2 divisorial blow ups with discrepancies $1 / 3$. By (9.4)(3), we also see that $\sum_{Q \in \bar{X}_{i}}$ $\left(\operatorname{aw}\left(\bar{X}_{i}, Q\right)-1\right)=1$ for $i=1$ and 2. As in the proof of (9.9), these are not isomorphic over $X$.

For the last part, we use $\pi_{1}$ as the first blow up. By (9.4)(3), $\bar{X}$ is non Gorenstein only at the origin of

$$
U_{2}=\left\{\bar{u}^{2}+\bar{x}^{3}+\bar{z}^{2}+\bar{x} \bar{y}^{4} \lambda\left(\bar{y}^{4}\right)+\bar{y}^{6} \mu\left(\bar{y}^{4}\right)=0\right\} / \mathbb{Z}_{4}(2,1,1,3)
$$

Since $\bar{x}^{3}+\bar{x} \bar{y}^{4} \lambda(0)+\bar{y}^{6} \mu(0)$ can not be a square, (7.4) shows that there is a unique divisor with discrepancy $1 / 4$ over $\bar{X}_{1}$. Thus there are exactly 2 divisors with discrepancies $1 / 3$ over $X$ as in the proof of (9.9).

9.15. Proposition. If $v^{\prime}=\left(j^{\prime}, \sigma^{\prime}\right)$ is a maximal element in $\mathscr{W}_{1 / 3}$, then $v^{\prime}>$ $v_{1,1}$ or $v^{\prime}>v_{1,2}$.

Proof. As in the proof of (9.10), we may assume that $\sigma^{\prime}=\sigma_{1,1}$. We shall derive a contradiction assuming that $v^{\prime} \Varangle_{v_{1,1}}$ and $v^{\prime} \ngtr v_{1,2}$. Let $\chi:\left(x^{\prime}, y^{\prime}, z^{\prime}, u^{\prime}\right) /$ $\mathbb{Z}_{3} \rightarrow(x, y, z, u) / \mathbb{Z}_{3}$ be a liftable automorphism such that $\chi \circ j^{\prime}=j$. Then we have $\sigma^{\prime}-w t\left(\chi^{*}(y)\right)=\sigma^{\prime}-w t\left(\chi^{*}(z)\right)=1 / 3$ by our assumption, so that $\sigma^{\prime}-w t\left(\chi^{*}\left(y z^{2}\right)\right)=$ 1. Thus we get $\sigma^{\prime}-w t\left(\varphi^{\prime}\right)=1$ for the defining equation $\varphi^{\prime}=\chi^{*}(\varphi)$ of $j^{\prime}$. This is a contradiction since $d\left(v^{\prime}\right)=1 / 3$.

9.16. By (9.14) and (9.15), we see that (4.1) and (4.4) hold if $X$ is of type (cD/3-2). By (9.14), we also see that (4.5) and (4.6) hold. Figure 3(9.B) shows the elements of $\mathscr{W}_{1 / 3} / \sim$ and their relations. 


\section{§ 9.C. Terminal singularities of type (cD/3-3)}

9.17. In this subsection, we assume that $X$ has a standard embedding

$$
\begin{gathered}
j: X \simeq\left\{u^{2}+x^{3}+y^{3}+x y z^{3} \alpha\left(z^{3}\right)+x z^{4} \beta\left(z^{3}\right)+y z^{5} \gamma\left(z^{3}\right)+z^{6} \delta\left(z^{3}\right)=0\right\} \\
/ \mathbb{Z}_{3}(2,1,1,0) \subseteq(x, y, z, u) / \mathbb{Z}_{3}(2,1,1,0)
\end{gathered}
$$

where $\alpha\left(z^{3}\right), \beta\left(z^{3}\right), \gamma\left(z^{3}\right), \delta\left(z^{3}\right) \in \mathbb{C}\left\{z^{3}\right\}$. We denote the power series expansion of $\alpha\left(z^{3}\right)$, etc. by $\alpha\left(z^{3}\right)=\alpha_{0}+\alpha_{1} z^{3}+\alpha_{2} z^{6}+\alpha_{3} z^{9}+\cdots$, etc. Here we consider the system of equations

$$
x^{3}+x \beta_{0}+\delta_{0}=0, \quad 3 x^{2}+\beta_{0}=0, \quad \alpha_{0} x+\gamma_{0}=0, \quad \beta_{1} x+\delta_{1}=0
$$

in $x$, and divide the case whether $(*)$ has a solution or not. As we shall see later, the $\left(j, \sigma_{1,1}\right)$-blow up of $X$ is divisorial if $(*)$ has no solutions. But if $(*)$ has a solution, then the $\left(j, \sigma_{1,1}\right)$-blow up of $X$ is not divisorial and we need another pseudo weighted valuation for our study of this case.

9.18. Under the notation and the assumption (9.17), we first assme that $(*)$ has no solutions in $x$. We shall study this case in (9.19)-(9.22).

9.19. Proposition. For the standard embedding $j$, we have

$$
\mathscr{W}_{1 / 3}(j)=\left\{(j, \sigma) \mid \sigma=\sigma_{0} \text { or } \sigma_{1,1}\right\}
$$

In particular, $v_{1,1}=\left(j, \sigma_{1,1}\right)$ is the unique maximal element in $\mathscr{W}_{1 / 3}(j)$.

Proof. By (9.2), it is enough to prove that $\left(j, \sigma_{2}\right) \notin \mathscr{W}_{1 / 3}(j)$. If $\left(j, \sigma_{2}\right) \in$ $\mathscr{W}_{1 / 3}(j)$, then (3.9.1) implies that $\sigma_{2}-w t(\varphi)=4$. Thus we get $\beta_{0}=\gamma_{0}=\delta_{0}=\delta_{1}=0$. This is a contradiction since $(*)$ has a solution $x=0$.

9.20. Theorem. The $v_{1,1}$-blow up $\pi: \bar{X} \rightarrow X$ is divisorial with discrepancy $1 / 3$ and $\sum_{Q \in \bar{X}}(\operatorname{aw}(\bar{X}, Q)-1)=1$. The exceptional divisor $E$ of $\pi$ is the unique divisor with discrepancy $1 / 3$ over $X$.

Proof. Since (*) has no solutions, (9.4)(2) implies that $\pi$ is divisorial with discrepancy $1 / 3$. By $(9.4)(3)$, we have $\sum_{Q \in \bar{X}}(\operatorname{aw}(\bar{X}, Q)-1)=1$ and $\bar{X}$ is non Gorenstein only at the origin $Q$ of

$$
\begin{aligned}
U_{2}= & \left\{\bar{u}^{2}+\bar{x}^{3}+\bar{y}^{2}+\bar{x} \bar{y} \bar{z}^{3} \alpha\left(\bar{y} \bar{z}^{3}\right)+\bar{x} \bar{z}^{4} \beta\left(\bar{y} \bar{z}^{3}\right)+\bar{y} \bar{z}^{5} \gamma\left(\bar{y} \bar{z}^{3}\right)+\bar{z}^{6} \delta\left(\bar{y} \bar{z}^{3}\right)=0\right\} \\
& / \mathbb{Z}_{4}(2,3,3,1) .
\end{aligned}
$$


As $x^{3}+x z^{4} \beta_{0}+z^{6} \delta_{0}$ is not a square, (7.4) implies that there is exactly one divisor $F$ with discrepancy $1 / 4$ over $Q$ and $F$ is obtained by the weighted blow up with weight $\frac{1}{4}(2,5,1,3)$ over $U_{2}$. We denote this blow up by $\nu: Z \rightarrow \bar{X}$. Since $E$ is defined by $\bar{y}=0$ in $U_{2}$, (3.5) implies that $\nu^{*}(E)=\nu^{-1}[E]+\frac{5}{4} F$. By (5.3), we see that $a(F, X)$ $=1 / 4+5 / 4 \cdot 1 / 3=2 / 3$. Thus we know that $E$ is the unique divisor with discrepancy $1 / 3$ over $X$.

9.21. Proposition. If $v^{\prime}=\left(j^{\prime}, \sigma^{\prime}\right)$ is a maximal element in $\mathscr{W}_{1 / 3}$, then $v^{\prime}>$ $v_{1,1}$.

Proof. As in the proof of (9.10), we may assume that $\sigma^{\prime}=\sigma_{1,1}$ or $\sigma_{2}$. We shall derive a contradiction assuming that $v^{\prime} \ngtr_{v_{1,1}}$. Let $\chi:\left(x^{\prime}, y^{\prime}, z^{\prime}, u^{\prime}\right) / \mathbb{Z}_{3} \rightarrow(x, y, z$, $u) / \mathbb{Z}_{3}$ be a liftable automorphism such that $\chi \circ j^{\prime}=j$ and $\varphi^{\prime}=\chi^{*}(\varphi)$ be the defining equation of $j^{\prime}$. By our assumption, we see that $z^{\prime} \in \chi^{*}(y)$. This implies that $z^{\prime 3} \in$ $\varphi^{\prime}$, so we have $\sigma^{\prime}-w t\left(\varphi^{\prime}\right)=1$. This contradicts the fact that $d\left(v^{\prime}\right)=1 / 3$.

9.22. By (9.20) and (9.21), we see that (4.1), (4.4), (4.5) and (4.6) hold under the assumption (9.18). Figure 3(9.C.a) shows the elements of $\mathscr{W}_{1 / 3} / \sim$ and their relations.

9.23. Under the notation and assumption (9.17), we next assume that $(*)$ has a solution $x=\xi \in \mathbb{C}$. Then $x=\xi$ is a double root of $x^{3}+\beta_{0} x+\delta_{0}=0$, so we can write $x^{3}+\beta_{0} x z^{4}+\delta_{0} z^{6}=\left(x-\xi z^{2}\right)^{2}\left(x+2 \xi z^{2}\right)$. This leads the automorphism $\chi_{1}:(x$, $y, z, u) / \mathbb{Z}_{3} \rightarrow\left(x_{1}, y_{1}, z_{1}, u_{1}\right) / \mathbb{Z}_{3}$ defined by

$$
\chi_{1}^{*}\left(x_{1}\right)=x-\xi z^{2}, \quad \chi_{1}^{*}\left(y_{1}\right)=y, \quad \chi_{1}^{*}\left(z_{1}\right)=z \quad \text { and } \quad \chi_{1}^{*}\left(u_{1}\right)=u,
$$

and the embedding $j_{1}=\chi_{1} \circ j: X \hookrightarrow\left(x_{1}, y_{1}, z_{1}, u_{1}\right) / \mathbb{Z}_{3}(2,1,1,3)$. The defining equation of $j_{1}$ is

$$
\varphi_{1}=u_{1}^{2}+x_{1}^{3}+3 \xi x_{1}^{2} z_{1}^{2}+y_{1}^{3}+x_{1} y_{1} z_{1}^{3} \alpha^{\prime}\left(z_{1}^{3}\right)+x_{1} z_{1}^{7} \beta^{\prime}\left(z_{1}^{3}\right)+y_{1} z_{1}^{8} \gamma^{\prime}\left(z_{1}^{3}\right)+z_{1}^{12} \delta^{\prime}\left(z_{1}^{3}\right),
$$

where $\alpha^{\prime}\left(z^{3}\right)=\alpha\left(z^{3}\right), \beta^{\prime}\left(z^{3}\right)=\left(\beta\left(z^{3}\right)-\beta_{0}\right) / z^{3}, \gamma^{\prime}\left(z^{3}\right)=\left(\xi \alpha\left(z^{3}\right)+\gamma\left(z^{3}\right)\right) / z^{3}, \delta^{\prime}\left(z^{3}\right)$ $=\left[\left(\beta\left(z^{3}\right)-\beta_{0}\right) \xi+\left(\delta\left(z^{3}\right)-\delta_{0}\right)\right] / z^{6}$. As before, we denote the power series expansion of $\alpha^{\prime}\left(z^{3}\right)$, etc. by $\alpha_{0}^{\prime}+\alpha_{1}^{\prime} z^{3}+\alpha_{2}^{\prime} z^{6}+\cdots$, etc. We shall study this case in (9.24)-(9.28).

9.24. Proposition. For the embedding $j_{1}$, we have

$$
\mathscr{W}_{1 / 3}\left(j_{1}\right)=\left\{\left(j_{1}, \sigma\right) \mid \sigma=\sigma_{0}, \sigma_{1,1} \text { or } \sigma_{2}\right\}
$$


In particular, $v_{2}^{\prime}=\left(j_{1}: \sigma_{2}\right)$ is the unique maximal element in $\mathscr{W}_{1 / 3}\left(j_{1}\right)$.

9.25. Theorem. The $v_{2}^{\prime}$-blow up $\pi^{\prime}: \bar{X}^{\prime} \rightarrow X$ is divisorial with discrepancy $1 / 3$ and $\Sigma_{Q \in \bar{X}^{\prime}}\left(\operatorname{aw}\left(\bar{X}^{\prime}, Q\right)-1\right)=0$. There are exactly 2 divisors with discrepancies $1 / 3$ over $X$.

Proof. Since the defining equation $\varphi_{1}$ satisfies (9.3.4), we can apply (9.5) and see that $\pi^{\prime}$ is divisorial with discrepancy $1 / 3$ and that $\Sigma_{Q \in \bar{X}^{\prime}}\left(\operatorname{aw}\left(\bar{X}^{\prime}, Q\right)-1\right)=0$.

Each point of $\bar{X}^{\prime}$ has index $\leq 3$ except at the origin $Q_{1}$ of the $x_{1}$-chart $U_{1}$ and $\bar{X}^{\prime}$ is isomorphic to $(\bar{y}, \bar{z}, \bar{u}) / \mathbb{Z}_{5}(1,4,4)$ near $Q_{1}$. We can resolve $Q_{1} \in \bar{X}^{\prime}$ by using (5.1) and get a projective birational morphism $\nu: Z \rightarrow \bar{X}^{\prime}$ such that $K_{Z}=\nu^{*}\left(K_{\bar{X}^{\prime}}\right)$ $+\sum_{i=1}^{4} \frac{i}{5} F_{i}$, where $\sum_{i=1}^{4} F_{i}$ is the exceptional divisor of $\nu$ over $Q_{1}$. Let $E^{\prime}$ be the exceptional divisor of $\pi^{\prime}$. By (9.5) and (5.1), we have

$$
\nu^{*}\left(E^{\prime}\right)=\nu^{-1}\left[E^{\prime}\right]+\frac{2}{5} F_{1}+\frac{4}{5} F_{2}+\frac{6}{5} F_{3}+\frac{3}{5} F_{4} .
$$

Using (5.3), we see that $a\left(F_{1}, X\right)=1 / 3$ and $a\left(F_{i}, X\right) \geq 2 / 3$ for $i \geq 2$. Therefore $E^{\prime}$ and $F_{1}$ are the divisors with discrepancies $1 / 3$ over $X$.

9.26. Remark. Thus we know that there are exactly 2 divisors with discrepancies $1 / 3$ over $X$. One of them is obtained as the exceptional divisor of the $v_{2}^{\prime}$-blow up. The other one is obtained as the exceptional divisor of the weighted blow up $\pi: \bar{X} \rightarrow X$ associated to $\left(j, \sigma_{1,1}\right) \sim\left(j_{1}, \sigma_{1,1}\right)$. This is not divisorial since $\bar{X}$ has non-terminal singularities.

9.27. Proposition. If $v^{\prime}=\left(j^{\prime}, \sigma^{\prime}\right)$ is a maximal element in $\mathscr{W}_{1 / 3}$, then $v^{\prime}>$ $v_{2}^{\prime}$.

Proof. We may assume that $\sigma^{\prime}=\sigma_{1,1}$ or $\sigma_{2}$ as in the proof of (9.10). By (9.21), we see that $v^{\prime}>\left(j, \sigma_{1,1}\right) \sim\left(j_{1}, \sigma_{1,1}\right)$.

If $\sigma^{\prime}=\sigma_{1,1}$, then (5.5) shows that $v^{\prime} \sim\left(j_{1}, \sigma_{1,1}\right)$ is not maximal.

Let $\sigma^{\prime}=\sigma_{2}$ and let $\chi:\left(x^{\prime}, y^{\prime}, z^{\prime}, u^{\prime}\right) / \mathbb{Z}_{3} \rightarrow\left(x_{1}, y_{1}, z_{1}, u_{1}\right) / \mathbb{Z}_{3}$ be a liftable automorphism such that $\chi \circ j^{\prime}=j_{1}$. Since $v^{\prime}>\left(j_{1}, \sigma_{1,1}\right)$, we have

$$
\sigma^{\prime}-w t s\left(\chi^{*}\left(x_{1}, y_{1}, z_{1}, u_{1}\right)\right) \geq \frac{1}{3}(2,4,1,3) \text {. }
$$

Thus we have to show that $\sigma^{\prime}-w t\left(\chi^{*}(x)\right) \geq 5 / 3$ and $\sigma^{\prime}-w t\left(\chi^{*}(u)\right) \geq 2$. Since $d\left(v^{\prime}\right)=1 / 3$, (3.9.1) shows that $\sigma^{\prime}-w t\left(\varphi^{\prime}\right)=4$ where $\varphi^{\prime}=\chi^{*}\left(\varphi_{1}\right)$ is the defining equation of $j^{\prime}$. Let

$$
p=\chi^{*}\left(x_{1}\right), \quad q=\chi^{*}\left(y_{1}\right), \quad r=\chi^{*}\left(z_{1}\right), \quad s=\chi^{*}\left(u_{1}\right) \in \mathbb{C}\left\{x^{\prime}, y^{\prime}, z^{\prime}, u^{\prime}\right\} .
$$


If $z^{\prime 3} \in s$, then $z^{\prime 6} \in \varphi^{\prime}$, so we have $\sigma^{\prime}-w t\left(\varphi^{\prime}\right) \leq 2$, which is a contradiction. Hence $z^{\prime 3} \not s$ and we get $\sigma^{\prime}-w t(s) \geq 2$. Let $p=a x^{\prime}+b z^{\prime 2}+$ (other terms) and $r=c y^{\prime}+d z^{\prime}+$ (other terms). Since $\chi$ is an automorphism, $a \neq 0, d \neq 0$. Since $\sigma^{\prime}-w t\left(\varphi^{\prime}\right)=4$, the coefficients of $x^{\prime} z^{\prime 4}$ and $z^{\prime 6}$ in $\varphi^{\prime}$ are both zero, so that we have $a b(3 b+6 \xi d)=b^{2}(b$ $\left.+3 \xi d^{2}\right)=0$. These equalities imply that $b=0$ which shows $\sigma^{\prime}-w t(p) \geq 5 / 3$. Thus we know that $v^{\prime}>v_{2}^{\prime}$.

9.28. By (9.25), (9.26) and (9.27), we see that (4.1), (4.4), (4.5) and (4.6) hold under the assumption (9.23). Figure 3(9.C.b) shows the elements of $\mathscr{W}_{1 / 3} /$ $\sim$ and their relations.

9.29. By (9.22) and (9.28), we complete the proof of (4.1), (4.4), (4.5) and (4.6) if $X$ is of type $(\mathrm{cD} / 3-3)$.

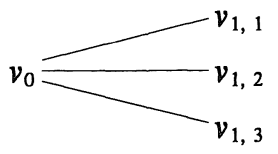

(9.A)

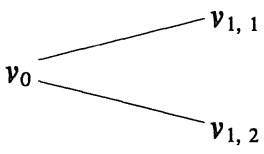

(9.B)

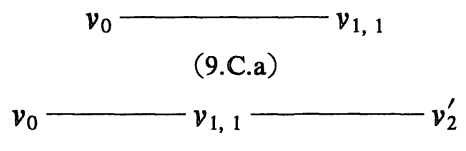

(9.C.b)

Figure 3. $\mathscr{W}_{1 / 3}$ for $(\mathrm{cD} / 3)$ type terminal singularities

\section{$\S 10$. Terminal Singularities of Type ( $c E / 2)$}

10.1. Let $X$ be a germ of a 3-dimensional terminal singularity of type (cE/2). By (2.3), there is a standard embedding

$$
\begin{aligned}
j: X & \simeq\left\{u^{2}+x^{3}+g(y, z) x+h(y, z)=0\right\} / \mathbb{Z}_{2}(0,1,1,1) \\
& \subseteq(x, y, z, u) / \mathbb{Z}_{2}(0,1,1,1)
\end{aligned}
$$

where $g(y, z), h(y, z) \in(y, z)^{4} \mathbb{C}\{y, z\}$ and the degree 4 part $h_{\operatorname{deg}} 4(y, z)$ of $h(y, z)$ is not zero. In this case, we have $\operatorname{aw}(X)=3$. We shall denote the defining equation as $\varphi=u^{2}+x^{3}+g(y, z) x+h(y, z)$, also we denote the power series expansion of $g(y, z)$ and $h(y, z)$ as $g(y, z)=\sum_{i, j} a_{i, j} y^{i} z^{j}$ and $h(y, z)=\sum_{i, j} b_{i, j} y^{i} z^{j}$ respectively.

As before, we first determine all the possible weights $\sigma^{\prime}$ such that $\left(j^{\prime}, \sigma^{\prime}\right) \in \mathscr{W}_{1 / 2}$ for some embedding $j^{\prime}: X \hookrightarrow\left(x^{\prime}, y^{\prime}, z^{\prime}, u^{\prime}\right) / \mathbb{Z}_{2}(0,1,1,1)$.

10.2. Lemma. Let $j^{\prime}: X \hookrightarrow\left(x^{\prime}, y^{\prime}, z^{\prime}, u^{\prime}\right) / \mathbb{Z}_{2}(0,1,1,1)$ be an arbitrary embedding and let $\varphi^{\prime}$ be the defining equation of $j^{\prime}$. Then, after a permutation of 
coordinates if necessary, $u^{\prime 2}, x^{\prime 3}$ and some degree 4 terms in $y^{\prime}, z^{\prime}$ appear in $\varphi^{\prime}$. Furthermore, if $\left(j^{\prime}, \sigma^{\prime}\right) \in \mathscr{W}_{1 / 2}$, then the following holds :

(1) If $y^{\prime 4} \in \varphi^{\prime}$, then $\sigma^{\prime}=\frac{1}{2}(2,1,1,1), \frac{1}{2}(2,1,1,3), \frac{1}{2}(2,3,1,3), \frac{1}{2}(4,3,1,5)$, $\frac{1}{2}(4,3,1,7)$ or $\frac{1}{2}(6,5,1,9)$.

(2) If $y^{\prime 3} z^{\prime} \in \varphi^{\prime}$, then $\sigma^{\prime}=\frac{1}{2}(2,1,1,1), \frac{1}{2}(2,1,1,3), \frac{1}{2}(2,3,1,3), \frac{1}{2}(2,1,3$, 3) or $\frac{1}{2}(4,3,1,5)$.

(3) If $y^{\prime 2} z^{\prime 2} \in \varphi^{\prime}$, then $\sigma^{\prime}=\frac{1}{2}(2,1,1,1), \frac{1}{2}(2,1,1,3), \frac{1}{2}(2,3,1,3)$ or $\frac{1}{2}(2,1$, $3,3)$. In particular, if the degree 4 part in $y^{\prime}$ and $z^{\prime}$ of $\varphi^{\prime}$ does not have a triple or a 4-ple factor, then $\sigma^{\prime}=\frac{1}{2}(2,1,1,1), \frac{1}{2}(2,1,1,3), \frac{1}{2}(2,3,1,3)$ or $\frac{1}{2}(2,1,3,3)$.

Proof. The first part is obvious. Let $w=\sigma^{\prime}-w t$ and $v^{\prime}=\left(j^{\prime}, \sigma^{\prime}\right)$. If $u^{\prime 2}, x^{\prime 3}, y^{\prime 4}$ $\in \varphi^{\prime}$, then we see that $2 w\left(u^{\prime}\right) \geq w\left(\varphi^{\prime}\right), 3 w\left(x^{\prime}\right) \geq w\left(\varphi^{\prime}\right)$ and $4 w\left(y^{\prime}\right) \geq w\left(\varphi^{\prime}\right)$. Since $d\left(v^{\prime}\right)=1 / 2$, it follows from (3.9.1) that

$$
1 / 2=w\left(x^{\prime}\right)+w\left(y^{\prime}\right)+w\left(z^{\prime}\right)+w\left(u^{\prime}\right)-w\left(\varphi^{\prime}\right)-1 \geq w\left(z^{\prime}\right)+w\left(\varphi^{\prime}\right) / 12-1 .
$$

Since $\sigma^{\prime} \in \mathbb{Z}^{4}+\frac{1}{2}(0,1,1,1) \mathbb{Z}$ and since $w\left(\varphi^{\prime}\right) \in \mathbb{Z}$, we see that $w\left(x^{\prime}\right) \equiv 0, w\left(y^{\prime}\right) \equiv$ $w\left(z^{\prime}\right) \equiv w\left(u^{\prime}\right) \equiv 1 / 2 \bmod \mathbb{Z}$. So the above inequality shows that $w\left(z^{\prime}\right)=1 / 2$ and $w\left(\varphi^{\prime}\right) \leq 12$. The rest follows from case by case analysis. For example, if $w\left(\varphi^{\prime}\right)=$ 9 , then we have $w\left(x^{\prime}\right) \geq 3, w\left(y^{\prime}\right) \geq 5 / 2, w\left(u^{\prime}\right) \geq 9 / 2$. Since $\sigma^{\prime}-w t\left(x^{\prime} y^{\prime} z^{\prime} u^{\prime}\right)=21 / 2$, these inequalities must be equalities. Therefore we have $\sigma^{\prime}=\frac{1}{2}(6,5,1,9)$. We can prove other cases similarly.

We define

$$
\begin{gathered}
\sigma_{0}=\frac{1}{2}(2,1,1,1), \sigma_{1}=\frac{1}{2}(2,1,1,3), \sigma_{2,1}=\frac{1}{2}(2,3,1,3), \sigma_{2,2}=\frac{1}{2}(2,1,3,3), \\
\sigma_{3}=\frac{1}{2}(4,3,1,5), \quad \sigma_{4}=\frac{1}{2}(4,3,1,7) \text { and } \sigma_{5}=\frac{1}{2}(6,5,1,9) .
\end{gathered}
$$

10.3. Let $j^{\prime}: X \hookrightarrow\left(x^{\prime}, y^{\prime}, z^{\prime}, u^{\prime}\right) / \mathbb{Z}_{2}(0,1,1,1)$ be an embedding and let $\varphi^{\prime}$ be the defining equation of $j^{\prime}$. We define $\tau$-wt $\left(y^{\prime}\right)=3 / 2, \tau-w t\left(z^{\prime}\right)=1 / 2$ and $\tau^{\prime}-w t\left(y^{\prime}\right)$ $=5 / 2, \tau^{\prime}-w t\left(z^{\prime}\right)=1 / 2$. We shall consider the following conditions on $\varphi^{\prime}$. (See (3.4) for the definition of $I^{\sigma}, 1$ (4) etc.)

(10.3.1) $\varphi^{\prime} \equiv u^{\prime 2}+x^{\prime 3}+a_{0,4} z^{\prime 4} x^{\prime}+b_{0,6} z^{\prime 6}+y^{\prime} z^{\prime 3} \bmod I^{\sigma_{2,1}}$ (4).

(10.3.2) $\varphi^{\prime} \equiv\left(u^{\prime 2}+x^{\prime 3}+a_{0,4} z^{\prime 4} x^{\prime}+b_{0,6} z^{\prime 6}\right)+y^{\prime} z^{\prime 3}\left(a_{1,3} x^{\prime}+b_{1,5} z^{\prime 2}\right)+z^{\prime 6}\left(a_{0,6} x^{\prime}\right.$ $\left.+b_{0,8} z^{\prime 2}\right)+y^{\prime 2} z^{\prime 2} \bmod I^{\sigma_{2,1}}(5)$ for some $a_{i, j}, b_{i, j} \in \mathbb{C}$.

(10.3.3) $\varphi^{\prime} \equiv\left(u^{\prime 2}+x^{\prime 3}+a_{0,4} z^{\prime 4} x^{\prime}+b_{0,6} z^{\prime 6}\right)+y^{\prime} z^{\prime 3}\left(a_{1,3} x^{\prime}+b_{1,5} z^{\prime 2}\right)+z^{\prime 6}\left(a_{0,6} x^{\prime}\right.$ $\left.+b_{0,8} z^{\prime 2}\right) \bmod I^{\sigma_{2,1}}(5)$ for some $a_{i, j}, b_{i, j} \in \mathbb{C}$, and $y^{\prime 3} z^{\prime}$ or $y^{\prime 4} \in \varphi^{\prime}$.

(10.3.4) $\varphi^{\prime} \equiv u^{\prime 2}+\lambda x^{\prime 2} z^{\prime 2}+g_{\tau-w t=3}\left(y^{\prime}, z^{\prime}\right) x^{\prime}+h_{\tau-w t=5}\left(y^{\prime}, z^{\prime}\right) \bmod I^{\sigma_{3}}(6)$ for some $\lambda \in \mathbb{C}, g\left(y^{\prime}, z^{\prime}\right), h\left(y^{\prime}, z^{\prime}\right) \in \mathbb{C}\left\{y^{\prime}, z^{\prime}\right\}$, and $x^{\prime 3} \in \varphi^{\prime}$.

(10.3.5) $\varphi^{\prime} \equiv\left(\alpha x^{\prime} z^{\prime}+\beta y^{\prime} z^{\prime 2}+\gamma z^{\prime 5}\right) u^{\prime}+x^{\prime 3}+g_{\tau-w t=4}\left(y^{\prime}, z^{\prime}\right) x^{\prime}+h_{\tau-w t=6}\left(y^{\prime}, z^{\prime}\right) \bmod$ 
$I^{\sigma_{4}}(7)$ for some $\alpha, \beta, \gamma \in \mathbb{C}, g\left(y^{\prime}, z^{\prime}\right), h\left(y^{\prime}, z^{\prime}\right) \in \mathbb{C}\left\{y^{\prime}, z^{\prime}\right\}$, and $u^{\prime 2} \in \varphi^{\prime}$.

(10.3.6) $\varphi^{\prime} \equiv u^{\prime 2}+x^{\prime 3}+g_{\tau^{\prime}-w t=6}\left(y^{\prime}, z^{\prime}\right) x^{\prime}+h_{\tau^{\prime}-w t=9}\left(y^{\prime}, z^{\prime}\right) \bmod I^{\sigma_{5}}$ (10) for some $g\left(y^{\prime}, z^{\prime}\right), h\left(y^{\prime}, z^{\prime}\right) \in \mathbb{C}\left\{y^{\prime}, z^{\prime}\right\}$, and $y^{\prime 4} \in \varphi^{\prime}$.

The proofs for the following propositions (10.4)-(10.7) are almost the same as the one for (9.4) and (9.5). So we shall omit their proofs.

10.4. Proposition. Let $\pi: \bar{X} \rightarrow X$ be the $\left(j^{\prime}, \sigma_{2,1}\right)$-blow up and let $E$ be the exceptional divisor of $\pi$. Then the following holds :

(1) If (10.3.1) or (10.3.2) holds, then $\pi$ is divisorial with discrepancy $1 / 2$.

(2) If (10.3.3) holds, then $\bar{X}$ has only canonical singularities and $E$ is irreducible. Furthermore, if the system of equations

$$
x^{3}+\alpha_{0,4} z^{4} x+b_{0,6}=0, \quad 3 x^{2}+a_{0,4}=0, \quad a_{1,3} x+b_{1,5}=0, \quad a_{0,6} x+b_{0,8}=0
$$

has no solutions in $x$, then $\pi$ is divisorial with discrepancy $1 / 2$.

(3) Assume that (10.3.1), (10.3.2) or (10.3.3) holds. In case (10.3.3), we further assume that $(\dagger)$ has no solutions. Then the origin of the $y^{\prime}$-chart of $\bar{X}$ is the unique non Gorenstein point and it is of type $(\mathrm{cD} / 3)$.

10.5. Proposition. Assume that $\varphi^{\prime}$ satisfies (10.3.4). Let $\pi: \bar{X} \rightarrow X$ be the $\left(j^{\prime}, \sigma_{3}\right)$-blow up and let $E$ be the exceptional divisor of $\pi$. Then the following holds :

(1) If $y^{\prime 3} z^{\prime} \in \varphi^{\prime}$, then $\pi$ is divisorial with discrepancy $1 / 2$.

(2) If $y^{\prime 3} z^{\prime} \notin \varphi^{\prime}, u^{\prime 2}+\lambda x^{\prime 2} z^{\prime 2}+g_{\tau-w t=3}\left(y^{\prime}, z^{\prime}\right) x^{\prime}+h_{\tau-w t=5}\left(y^{\prime}, z^{\prime}\right)$ is irreducible and reduced and $y^{\prime 4} \in \varphi$, then $\pi$ is also divisorial with discrepancy $1 / 2$.

(3) Under the above conditions of (1) or (2), $\bar{X}$ is Gorenstein outside two points. One of them is the origin $Q_{1}$ of the $x^{\prime}$-chart of $\bar{X}$ and it is isomorphic to $(\bar{y}, \bar{z}, \bar{u}) / \mathbb{Z}_{4}$ $(3,1,1)$. The other point is the origin $Q_{2}$ of the $y^{\prime}$-chart of $\bar{X}$ and it is isomorphic to $(\bar{x}, \bar{y}, \bar{u}) / \mathbb{Z}_{3}(1,1,2)$ in case (1) and $(\bar{x}, \bar{z}, \bar{u}) / \mathbb{Z}_{3}(1,1,2)$ in case (2). Moreover, $E$ is defined by $\bar{u}^{2}+\lambda \bar{z}^{2}+g_{\tau-w t=3}(\bar{y}, \bar{z})+h_{\tau-w t=5}(\bar{y}, \bar{z})=0$ near $Q_{1}$. Near $Q_{2}, E$ is defined by $\bar{y}=0$ in case (1) and $\bar{u}^{2}+\lambda \bar{x}^{2} \bar{z}^{2}+g_{\tau-w t=3}(1, \bar{z}) \bar{x}+h_{\tau-w t=5}(1, \bar{z})=0$ in case (2).

10.6. Proposition. Assume that $\varphi^{\prime}$ sotisfies (10.3.5). Let $\pi: \bar{X} \rightarrow X$ be the $\left(j^{\prime}, \sigma_{4}\right)$-blow up and let $E$ be the exceptional divisor of $\pi$. Then the following holds :

(1) If $(\alpha, \beta, \gamma) \neq(0,0,0)$, then $\pi$ is divisorial with discrepancy $1 / 2$.

(2) If $\alpha=\beta=\gamma=0$, then $\bar{X}$ has only canonical singularities and $E$ is irreducible. Moreover, $\bar{X}$ is terminal outside the $z^{\prime}$-chart of $\bar{X}$ and $\operatorname{Sing}(\bar{X})$ is isolated.

(3) $\bar{X}$ is Gorenstein outside the origin $Q$ of the $u^{\prime}$-chart of $\bar{X}$ and it is isomorphic to $(\bar{x}, \bar{y}, \bar{z}) / \mathbb{Z}_{7}(4,3,1) . \quad E$ is defined by $\left(\alpha \bar{x} \bar{z}+\beta \bar{y} \bar{z}^{2}+\gamma \bar{z}^{5}\right)+\bar{x}^{3}+g_{\tau-w t=4}(\bar{y}, \bar{z}) \bar{x}+$ $h_{\tau-w t=6}(\bar{y}, \bar{z})=0$ near $Q$. 
10.7. Proposition. Assume that $\varphi^{\prime}$ satisfies (10.3.6). Let $\pi: \bar{X} \rightarrow X$ be the $\left(j^{\prime}, \sigma^{\prime}\right)$-blow up and let $E$ be the exceptional divisor of $\pi$. Then $\pi$ is divisorial with discrepancy $1 / 2$ and $\bar{X}$ is Gorenstein outside two points. One of them is the origin $Q_{1}$ of the $y^{\prime}$-chart of $\bar{X}$ and it is isomorphic to $(\bar{x}, \bar{z}, \bar{u}) / \mathbb{Z}_{5}(4,4,1)$. The other point $Q_{2}$ is isomorphic to $\mathbb{C}^{3} / \mathbb{Z}_{3}(2,1,1) . \quad E$ is defined by $\bar{u}^{2}+\bar{x}^{3}+g_{\tau^{\prime}-w t=6}(1, \bar{z}) \bar{x}+$ $h_{\tau^{\prime}-w t=9}(1, \bar{z})=0$ near $Q_{1}$.

Now, we divide the case into several cases.

\section{§ 10.A. $h_{\operatorname{deg} 4}(y, z)$ has 4 distinct factors}

10.8. If $h_{\operatorname{deg} 4}(y, z)$ has 4 distinct factors, we may assume that the standard embedding $j$ is given so that $h_{\operatorname{deg} 4}(y, z)=y z\left(y+\lambda_{1} z\right)\left(y+\lambda_{2} z\right)$ for some $\lambda_{1}, \lambda_{2} \in \mathbb{C}$ by a linear change of $y$ and $z$. We shall treat this case in (10.9)-(10.13).

The following proposition is immediate from (10.2).

10.9. Proposition. For the standard embedding $j$, we have

$$
\mathscr{W}_{1 / 2}(j)=\left\{(j, \sigma) \mid \sigma=\sigma_{0}, \sigma_{1}, \sigma_{2,1} \text { or } \sigma_{2,2}\right\}
$$

In particular, $v_{2,1}=\left(j, \sigma_{2,1}\right)$ and $v_{2,2}=\left(j, \sigma_{2,2}\right)$ are the maximal elements in $\mathscr{W}_{1 / 2}(j)$.

10.10. We need two more pseudo weighted valuations, which naturally arise if we consider the $\left(j, \sigma_{1}\right)$-blow up of $X$. For each $i=1$ and 2 , let $\chi_{i}:(x, y, z, u) /$ $\mathbb{Z}_{2} \rightarrow\left(x_{i}, y_{i}, z_{i}, u_{i}\right) / \mathbb{Z}_{2}$ be the automorphism defined by

$$
\chi_{i}^{*}\left(x_{i}\right)=x, \quad \chi_{i}^{*}\left(y_{i}\right)=y+\lambda_{l} z, \quad \chi_{i}^{*}\left(z_{i}\right)=z \quad \text { and } \quad \chi_{i}^{*}\left(u_{i}\right)=u,
$$

and let $j_{i}=\chi_{i} \circ j: X \hookrightarrow\left(x_{i}, y_{i}, z_{i}, u_{i}\right) / \mathbb{Z}_{2}(0,1,1,1)$ be the embedding. Then their defining equations are

$$
\varphi_{i}=u_{i}^{2}+x_{i}^{3}+g_{i}\left(y_{i}, z_{i}\right) x_{i}+h_{i}\left(y_{i}, z_{i}\right) \text {, }
$$

where $g_{i}(y, z)=g\left(y-\lambda_{i} z, z\right), h_{i}(y, z)=h\left(y-\lambda_{i} z, z\right)$. In particular, the degree 4 part of $h_{i}(y, z)$ is $y z\left(y-\lambda_{i} z\right)\left(y-\mu_{i} z\right)$ where $\mu_{1}=\lambda_{1}-\lambda_{2}, \mu_{2}=\lambda_{2}-\lambda_{1}$. Let $v_{2,3}=$ $\left(j_{1}, \sigma_{2,1}\right), v_{2,4}=\left(j_{2}, \sigma_{2,1}\right)$, then $v_{2,3}, v_{2,4} \in \mathscr{W}_{1 / 2}$.

10.11. Theorem. For each $i=1,2,3$ and 4, the $v_{2, i}$-blow up $\pi_{i}: \bar{X}_{i} \rightarrow X$ is divisorial with discrepancy $1 / 2$ and $\sum_{Q \in \bar{X}_{i}}\left(\operatorname{aw}\left(\bar{X}_{i}, Q\right)-1\right)=1$. These $\pi_{i}$ are not mutually isomorphic over $X$. Furtheremore, there are exactly 4 divisors with dis- 
crepancies $1 / 2$ over $X$.

Proof. By $(10.4)(1), \pi_{1}$ is divisorial with discrepancy $1 / 2$. As terminal singularities of type $(\mathrm{cD} / 3)$ have axial weight 2 , we see from (10.4)(3) that $\sum_{Q \in \bar{X}_{1}}\left(\operatorname{aw}\left(\bar{X}_{1}, Q\right)-1\right)=1$. Similarly, $\pi_{2}, \pi_{3}$ and $\pi_{4}$ have the same properties.

Let $D$ be the $\mathbb{Q}$-Cartier Weil divisor on $X$ defined by $y=0$ and let $E_{i}$ be the exceptional divisor of $\pi_{i}(i=1,2,3,4)$. By (3.5), we have

$$
\pi_{1}^{*}(D)=\pi_{1}^{-1}[D]+\frac{3}{2} E_{1} \text { and } \pi_{i}^{*}(D)=\pi_{i}^{-1}[D]+\frac{1}{2} E_{i} \quad \text { if } i \neq 1 .
$$

Hence $\pi_{1}$ and $\pi_{i}(i \neq 1)$ are not isomorphic. Similarly $\pi_{1}, \pi_{2}, \pi_{3}$ and $\pi_{4}$ are not isomorphic over $X$.

In order to count the number of divisors with discrepancies $1 / 2$, we use $\pi_{1}$ as the first blow up. By (10.4) (3), the origin of the $z$-chart of $\bar{X}_{1}$ is the unique non Gorenstein point of $\bar{X}_{1}$ and it is of type (cD/3). By using the results in section 9, we see that there are at most 3 divisors with discrepancies $1 / 3$ over $\bar{X}_{1}$. By (5.3), there are at most 4 divisors with discrepancies $1 / 2$ over $X$ (including $E_{1}$ ). On the other hand we already have 4 divisors $E_{1}, \ldots, E_{4}$ with discrepancies $1 / 2$ over $X$.

10.12. Proposition. If $v^{\prime}=\left(j^{\prime}, \sigma^{\prime}\right) \in \mathscr{W}_{1 / 2}$ is maximal, then $v^{\prime}>v_{2, i}$ for some $i=1,2,3$ or 4 .

Proof. If $\sigma^{\prime}=\sigma_{0}$, then $v^{\prime} \sim\left(j, \sigma_{0}\right)$ is not maximal by (5.4). In case $\sigma^{\prime}=\sigma_{1}$, it is also easy to see that $v^{\prime} \sim\left(j, \sigma_{1}\right)$ is not maximal.

Thus we may assume that $\sigma^{\prime}=\sigma_{2,1}$ or $\sigma_{2,2}$ by (10.2). By symmetry, we assume that $\sigma^{\prime}=\sigma_{2,1}$. We shall show that $v^{\prime}>v_{2,3}$ or $v^{\prime}>v_{2,4}$ assuming that $v^{\prime} \ngtr v_{2,1}, v^{\prime} \ngtr$ $v_{2,2}$. Let $\chi:\left(x^{\prime}, y^{\prime}, z^{\prime}, u^{\prime}\right) / \mathbb{Z}_{2} \rightarrow(x, y, z, u) / \mathbb{Z}_{2}$ be a liftable automorphism such that $j=\chi \circ j^{\prime}$. Since $v^{\prime}>\left(j, \sigma_{1}\right)$, we have

$$
\sigma^{\prime}-w t s\left(\chi^{*}(x, y, z, u)\right) \geq \frac{1}{2}(2,1,1,3) .
$$

Thus our assumption implies that $\sigma^{\prime}-w t\left(\chi^{*}(y)\right)=\sigma^{\prime}-w t\left(\chi^{*}(z)\right)=1 / 2$. For the defining equation $\varphi^{\prime}=\chi^{*}(\varphi)$ of $j^{\prime}$, we have $\sigma^{\prime}-w t\left(\varphi^{\prime}\right)=3$ since $d\left(v^{\prime}\right)=1 / 2$. Hence $\sigma^{\prime}-w t\left(\chi^{*}\left(y+\lambda_{1} z\right)\right) \geq 3 / 2$ or $\sigma^{\prime}-w t\left(\chi^{*}\left(y+\lambda_{2} z\right)\right) \geq 3 / 2$. This proves that $v^{\prime}>v_{2,3}$ or $v^{\prime}>v_{2,4}$.

10.13. By (10.11) and (10.12), we see that (4.1), (4.4), (4.5) and (4.6) hold if $h_{\operatorname{deg} 4}(y, z)$ has 4 distinct factors. Figure 4(10.A) shows the elements of $\mathscr{W}_{1 / 2} / \sim$ and their relations. 


\section{§ 10.B. $h_{\operatorname{deg} 4}(y, z)$ has 1 double factor and 2 single factors}

10.14. If $h_{\mathrm{deg}} 4(y, z)$ has 1 double factor and 2 single factors, we may assume that the standard embedding $j$ is given so that $h_{\operatorname{deg} 4}(y, z)=y z^{2}(y+z)$ by a linear change in $y$ and $z$. We shall study this case in (10.15)-(10.19).

The following proposition easily follows from (10.2).

10.15. Proposition. For the standard embedding $j$, we have

$$
\mathscr{W}_{1 / 2}(j)=\left\{(j, \sigma) \mid \sigma=\sigma_{0}, \sigma_{1}, \sigma_{2,1} \text { or } \sigma_{2,2}\right\}
$$

In particular, $v_{2,1}=\left(j, \sigma_{2,1}\right)$ and $v_{2,2}=\left(j, \sigma_{2,2}\right)$ are the maximal elements in $\mathscr{W}_{1 / 2}(j)$.

10.16. As in (10.A), let $\chi_{1}:(x, y, z, u) / \mathbb{Z}_{2} \rightarrow\left(x_{1}, y_{1}, z_{1}, u_{1}\right) / \mathbb{Z}_{2}$ be the automorphism defined by

$$
\chi_{1}^{*}\left(x_{1}\right)=x, \quad \chi_{1}^{*}\left(y_{1}\right)=y+z, \quad \chi_{1}^{*}\left(z_{1}\right)=z \quad \text { and } \quad \chi_{1}^{*}\left(u_{1}\right)=u,
$$

and let $j_{1}=\chi_{1} \circ j: X \hookrightarrow\left(x_{1}, y_{1}, z_{1}, u_{1}\right) / \mathbb{Z}_{2}(0,1,1,1)$ be the embedding. The defining equation of $j_{1}$ is

$$
\varphi_{1}=u_{1}^{2}+x_{1}^{3}+g_{1}\left(y_{1}, z_{1}\right) x_{1}+h_{1}\left(y_{1}, z_{1}\right)
$$

where $g_{1}(y, z)=g(y-z, z), h_{1}(y, z)=h(y-z, z)$ so that degree 4 part of $h_{1}(y, z)$ is $y z^{2}(y-z)$. Then $v_{2,3}=\left(j_{1}, \sigma_{2,1}\right) \in \mathscr{W}_{1 / 2}$.

10.17. Theorem. For each $i=1,2$ and 3, the $v_{2, i}$-blow up $\pi_{i}: \bar{X}_{i} \rightarrow X$ is divisorial with discrepancy $1 / 2$ and $\sum_{Q \in \bar{X}_{i}}\left(\operatorname{aw}\left(\bar{X}_{i}, Q\right)-1\right)=1$. These $\pi_{i}$ are not mutually isomorphic over $X$. Furthermore, there are exactly 3 divisors with discrepancies $1 / 3$ over $X$.

Proof. By (10.4)(1), we see that each $\pi_{i}$ is divisorial with discrepancy $1 / 2$. By $(10.4)(3)$, we also see that $\sum_{Q \in \bar{X}_{i}}\left(\operatorname{aw}\left(\bar{X}_{i}, Q\right)-1\right)=1$. These $\pi_{i}$ are not isomorphic over $X$ as in the proof of (10.11).

In order to count the number of divisors with discrepancies $1 / 2$ over $X$, we shall use $\pi_{1}$ as the first blow up. By $(10.4)(3), \bar{X}_{1}$ has non Gorenstein point only at the origin of

$$
U_{2}=\left\{\bar{u}^{2}+\bar{x}^{3}+g\left(\bar{y}^{3 / 2}, \bar{y}^{1 / 2} \bar{z}\right) / \bar{y}^{2} \cdot \bar{x}+h\left(\bar{y}^{3 / 2}, \bar{y}^{1 / 2} \bar{z}\right) / \bar{y}^{3}=0\right\} / \mathbb{Z}_{3}(2,1,1,0)
$$


which is of type (cD/3-2). There are exactly 2 divisors with discrepancies $1 / 3$ over this point by (9.14). Thus we know that there are at most 3 divisors with discrepancies $1 / 2$ over $X$ by (5.3). On the other hand, we already know that there are at least 3 divisors with discrepancies $1 / 2$ over $X$.

We can prove the following by the same method as the proof of (10.12).

10.18. Proposition. If $v^{\prime}=\left(j^{\prime}, \sigma^{\prime}\right) \in \mathscr{W}_{1 / 2}$ is maximal, then $v^{\prime}>v_{2, i}$ for some $i=1,2$ or 3 .

10.19. By (10.17) and (10.18), we see that (4.1), (4.4), (4.5) and (4.6) hold if $h_{\operatorname{deg} 4}(y, z)$ has 1 double factor and 2 single factors. Figure 4(10.B) shows the elements of $\mathscr{W}_{1 / 2} / \sim$ and their relations.

\section{§ 10.C. $h_{\operatorname{deg} 4}(y, z)$ has 2 double factors}

10.20. If $h_{\operatorname{deg} 4}(y, z)$ has 2 double factors, we may assume that the standard embedding is given so that $h_{\operatorname{deg} 4}(y, z)=y^{2} z^{2}$ by a linear change of $y$ and $z$. We shall study this case in (10.21)-(10.24).

10.21. Proposition. For the standard embedding $j$, we have

$$
\mathscr{W}_{1 / 2}(j)=\left\{(j, \sigma) \mid \sigma=\sigma_{0}, \sigma_{1}, \sigma_{2,1} \text { or } \sigma_{2,2}\right\}
$$

In particular, $v_{2,1}=\left(j, \sigma_{2,1}\right)$ and $v_{2,2}=\left(j, \sigma_{2,2}\right)$ are the maximal elements in $\mathscr{W}_{1 / 2}(j)$.

10.22. Theorem. For each $i=1$ and 2 , the $v_{2, i}$-blow up $\pi_{i}: \bar{X}_{i} \rightarrow X$ is divisorial with discrepancy $1 / 2$ and $\sum_{Q \in \bar{X}_{i}}\left(\operatorname{aw}\left(\bar{X}_{i}, Q\right)-1\right)=1$. These $\pi_{1}$ and $\pi_{2}$ are not isomorphic over $X$. Furthermore, there are exactly 2 divisors with discrepancies $1 / 2$ over $X$.

Proof. By (10.4)(1), we see that $\pi_{1}$ and $\pi_{2}$ are both divisorial with discrepancies 1/2. By $(10.4)(3)$, we also see that $\sum_{Q \in \bar{X}_{i}}\left(\operatorname{aw}\left(\bar{X}_{i}, Q\right)-1\right)=1$. As in the proof of (10.11), $\pi_{1}$ and $\pi_{2}$ are not isomorphic over $X$.

For the last part, we shall use $\pi_{1}$ as the first blow up and let $E_{1}$ be the exceptional divisor of $\pi_{1}$. As in the proof of (10.17), there are 2 divisors $F_{1}$ and $F_{2}$ with discrepancies $1 / 3$ over the origin of $U_{2}$. These are obtained by the weighted blow up with weight $\tau_{1}=\frac{1}{3}(2,1,4,3)$ and $\tau_{2}=\frac{1}{3}(2,4,1,3)$ over $U_{3}$ respectively. Since $E_{1}$ is defined by $\bar{y}=0,(3.5)$ and (5.3) imply that $a\left(F_{1}, X\right)=1 / 3+1 / 2$. $4 / 3=1$ and $a\left(F_{2}, X\right)=1 / 3+1 / 2 \cdot 1 / 3=1 / 2$. Thus $E_{1}$ and $F_{2}$ are the divisors with discrepancies $1 / 2$ over $X$. 
We can easily prove the following as in the proof of (10.12).

10.23. Proposition. If $v^{\prime}=\left(j^{\prime}, \sigma^{\prime}\right) \in \mathscr{W}_{1 / 2}$ is maximal, then $v^{\prime}>v_{2,1}$ or $v^{\prime}>$ $v_{2,2}$.

10.24. By (10.22) and (10.23), we see that (4.1), (4.4), (4.5) and (4.6) hold if $h_{\operatorname{deg} 4}(y, z)$ has 2 double factors. Figure 4(10.C) shows the elements of $\mathscr{W}_{1 / 2} / \sim$ and their relations.

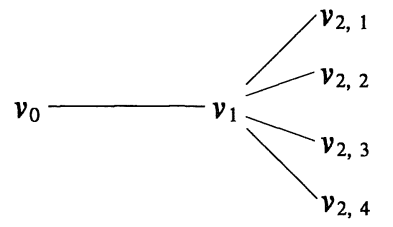

(10.A)

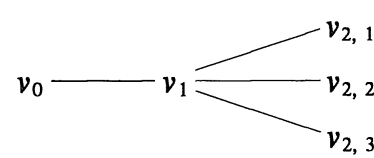

(10.B)

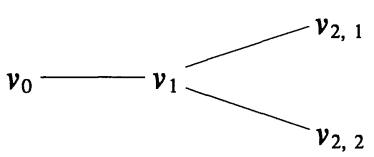

(10.C)

Figure 4. $\mathscr{W}_{1 / 2}$ for $(\mathrm{cE} / 2)$ type terminal singularities

\section{§ 10.D. $h_{\operatorname{deg} 4}(y, z)$ has a triple factor and a single factor}

10.25. In the case $h_{\operatorname{deg} 4}(y, z)$ has a triple factor and a single factor, we may assume that the standard embedding $j$ is given so that $h_{\operatorname{deg} 4}(y, z)=y^{3} z$ by a linear change of $y$ and $z$. As in (9.C), we first consider the system of algebraic equations

$$
x^{3}+a_{0,4} x+b_{0,6}=0, \quad 3 x^{2}+a_{0,4}=0, \quad a_{0,6} x+b_{0,8}=0, \quad a_{1,3} x+b_{1,5}=0
$$

in $x$ and divide the cases whether ( $\dagger$ ) has a solution or not.

10.26. We first assume that $(\dagger)$ has no solutions in $x$ and we shall study this case in (10.27)-(10.30).

10.27. Proposition. For the standard embedding $j$, we have

$$
\mathscr{W}_{1 / 2}(j)=\left\{(j, \sigma) \mid \sigma=\sigma_{0}, \sigma_{1}, \sigma_{2,1} \text { or } \sigma_{2,2}\right\}
$$

In particular, $v_{2,1}=\left(j, \sigma_{2,1}\right)$ and $v_{2,2}=\left(j, \sigma_{2,2}\right)$ are the maximal elements in $\mathscr{W}_{1 / 2}(j)$.

Proof. By (10.2), it is enough to prove that $\left(j, \sigma_{3}\right) \notin \mathscr{W}_{1 / 2}(j)$. If $\left(j, \sigma_{3}\right) \in$ $\mathscr{W}_{1 / 2}(j)$, then (3.9.1) implies $\sigma_{3}-w t(\varphi)=5$. Thus we get $a_{0,4}=b_{0,6}=b_{0,8}=b_{1,5}=0$. This is a contradiction since $(\dagger)$ has a solution $x=0$. 
10.28. Theorem. For each $i=1$ and 2, the $v_{2, i}$-blow up $\pi_{i}: \bar{X}_{i} \rightarrow X$ is divisorial with discrepancy $1 / 2$ and $\sum_{Q \in \bar{X}_{i}}\left(\operatorname{aw}\left(\bar{X}_{i}, Q\right)-1\right)=1$. These $\pi_{1}$ and $\pi_{2}$ are not isomorphic over $X$. Furthermore, there are exactly 2 divisors with discrepancies $1 / 2$ over $X$.

Proof. We see that $\pi_{1}$ (resp. $\pi_{2}$ ) is divisorial with discrepancy $1 / 2$ by (10.4) (2) (resp. $(10.4)(1))$. We also see that $\sum_{Q \in \bar{X}_{i}}\left(\operatorname{aw}\left(\bar{X}_{i}, Q\right)-1\right)=1$ by $(10.4)(3)$. Since singularities on $\bar{X}_{1}$ and $\bar{X}_{2}$ are different, $\pi_{1}$ and $\pi_{2}$ are not isomorphic over $X$.

We can prove the last part exactly by the same method as (10.22) using $\pi_{1}$ as the first blow up.

We can easily prove the following as in the proof of (10.12).

10.29. Proposition. If $v^{\prime}=\left(j^{\prime}, \sigma^{\prime}\right) \in \mathscr{W}_{1 / 2}$ is maximal, then $v^{\prime}>v_{2,1}$ or $v^{\prime}>$ $v_{2,2}$.

10.30. Thus, if $h_{\operatorname{deg} 4}(y, z)=y^{3} z$ and if $(\dagger)$ has no solutions in $x$, then (10.28) and (10.29) imply that (4.1), (4.4), (4.5) and (4.6) hold. Figure 5(10.D.a) shows the elements of $\mathscr{W}_{1 / 2} / \sim$ and their relations.

10.31. We next assume that $(\dagger)$ has a solution $x=\xi \in \mathbb{C}$ and we shall treat this case in (10.32)-(10.36).

In this case, $x^{3}+a_{0,4} x z^{4}+b_{0,6} z^{6}=\left(x-\xi z^{2}\right)^{2}\left(x+2 \xi z^{2}\right)$ as in (9.23). Let $\chi_{1}:(x$, $y, z, u) / \mathbb{Z}_{2} \rightarrow\left(x_{1}, y_{1}, z_{1}, u_{1}\right) / \mathbb{Z}_{2}$ be the automorphism defined by

$$
\chi_{1}^{*}\left(x_{1}\right)=x-\xi z^{2}, \quad \chi_{1}^{*}\left(y_{1}\right)=y, \quad \chi_{1}^{*}\left(z_{1}\right)=z \quad \text { and } \quad \chi_{1}^{*}\left(u_{1}\right)=u,
$$

and let $j_{1}=\chi_{1} \circ j: X \hookrightarrow\left(x_{1}, y_{1}, z_{1}, u_{1}\right) / \mathbb{Z}_{2}(0,1,1,1)$ be the embedding. Then the defining equation of $j_{1}$ is

$$
\varphi_{1}=u_{1}^{2}+x_{1}^{3}+3 \xi x_{1}^{2} z_{1}^{2}+g_{1}\left(y_{1}, z_{1}\right) x_{1}+h_{1}\left(y_{1}, z_{1}\right),
$$

where $g_{1}(y, z)=g(y, z)-a_{0,4} z^{4}, h_{1}(y, z)=\xi z^{2}\left(g(y, z)-a_{0,4} z^{4}\right)+(h(y, z)-$ $\left.b_{0,6} z^{6}\right)$. Since $x=\xi$ satisfies $(\dagger)$, we know that $\tau$-wt $\left(g_{1}(y, z)\right) \geq 3, \tau-w t\left(h_{1}(y, z)\right)$ $\geq 5$ if we denote $\tau$-wt $(y)=3 / 2, \tau$-wt $(z)=1 / 2$.

10.32. Proposition. For the embedding $j_{1}$, we have

$$
\mathscr{W}_{1 / 2}\left(j_{1}\right)=\left\{\left(j_{1}, \sigma\right) \mid \sigma=\sigma_{0}, \sigma_{1}, \sigma_{2,1}, \sigma_{2,2} \text { or } \sigma_{3}\right\}
$$

In particular, $v_{3}=\left(j_{1}, \sigma_{3}\right)$ and $v_{2,2}^{\prime}=\left(j_{1}, \sigma_{2,2}\right)$ are the maximal elements in $\mathscr{W}_{1 / 2}\left(j_{1}\right)$. 
10.33. Theorem. The $v_{3}$-blow up $\pi_{3}: \bar{X}_{3} \rightarrow X$ and the $v_{2,2}^{\prime}$-blow up $\pi_{2}: \bar{X}_{2} \rightarrow$ $X$ are both divisorial with discrepancies $1 / 2$ and these $\pi_{3}$ and $\pi_{2}$ are not isomorphic over $X$. We also have $\Sigma_{Q \in \bar{X}_{3}}\left(\operatorname{aw}\left(\bar{X}_{3}, Q\right)-1\right)=0$ and $\sum_{Q \in \bar{X}_{2}}\left(\operatorname{aw}\left(\bar{X}_{2}, Q\right)-1\right)=1$. Furthermore, there are exactly 3 divisors with discrepancies $1 / 2$ over $X$.

Proof. By (10.5)(1) (resp. (10.4)(1)), we see that $\pi_{3}$ (resp. $\pi_{2}$ ) is divisorial with discrepancy $1 / 2$. By $(10.5)(3)$ and $(10.4)(3)$, we also see that $\sum_{Q \in \bar{X}_{3}}\left(\operatorname{aw}\left(\bar{X}_{3}, Q\right)-1\right)=0$ and $\sum_{Q \in \bar{X}_{2}}\left(\operatorname{aw}\left(\bar{X}_{2}, Q\right)-1\right)=1$. These $\pi_{3}$ and $\pi_{2}$ are not isomorphic over $X$ since singularities of $\bar{X}_{2}$ and $\bar{X}_{3}$ are different.

In order to count the number of divisors with discrepancies $1 / 2$, we shall use $\pi_{3}$ as the first blow up. Let $E_{3}$ be the exceptional divisor of $\pi_{3}$. By (5.1), there is a projective birational morphism $\nu: Z \rightarrow \bar{X}_{3}$, which is a resolution of the origin $Q_{1}$ of the $x_{1}$-chart $U_{1}$ and the origin $Q_{2}$ of the $y_{1}$-chart $U_{2}$, such that $K_{Z}=\nu^{*}\left(K_{\bar{X}_{3}}\right)$ $+\sum_{i=1}^{3} \frac{i}{4} F_{i}+\sum_{j=1}^{2} \frac{j}{3} G_{j}$, where $\sum F_{i}$ (resp. $\sum G_{j}$ ) is the exceptional divisor of $\nu$ over $Q_{1}$ (resp. $Q_{2}$ ). By (10.5) (3) and (5.1), we have

$$
\nu^{*}\left(E_{3}\right)=\nu^{-1}\left[E_{3}\right]+\frac{1}{2} F_{1}+F_{2}+\frac{3}{2} F_{3}+\frac{1}{3} G_{1}+\frac{2}{3} G_{2} .
$$

Hence $a\left(F_{1}, X\right)=a\left(G_{1}, X\right)=1 / 2$ and $a\left(F_{2}, X\right), a\left(F_{3}, X\right), a\left(G_{2}, X\right) \geq 1$. Therefore $E_{3}, F_{1}$ and $G_{1}$ are the divisors with discrepancies $1 / 2$ over $X$.

10.34. Remark. Among these three divisors, two of them are obtained in (10.33). The remaining one is obtained as the exceptional divisor of the $v_{2,1}$-blow up $\pi_{2,1}: \bar{X}_{2,1} \rightarrow X$ where $v_{2,1}=\left(j, \sigma_{2,1}\right)$. This is not a divisorial blow up since $\bar{X}_{2,1}$ has non-terminal singularities.

10.35. Proposition. If $v^{\prime}=\left(j^{\prime}, \sigma^{\prime}\right) \in \mathscr{W}_{1 / 2}$ is maximal, then $v^{\prime}>v_{3}$ or $v^{\prime}>$ $v_{2,2}^{\prime}$.

Proof. As in the proof of (10.12), $v^{\prime}$ is not maximal if $\sigma^{\prime}=\sigma_{0}$ or $\sigma_{1}$. Hence we may assume $\sigma^{\prime}=\sigma_{2,1}$ or $\sigma_{3}$ by (10.2). We shall show that $v^{\prime}>v_{3}$ assuming that $v^{\prime} \ngtr$ $v_{2,2}^{\prime}$. Since $v_{2,2}^{\prime} \sim v_{2,2}$, (10.29) implies that $v^{\prime}>v_{2,1} \sim\left(j_{1}, \sigma_{2,1}\right)$. If $\sigma^{\prime}=\sigma_{2,1}$, then $v^{\prime} \sim\left(j_{1}, \sigma_{2,1}\right)$ is not maximal by (5.5). So we assume $\sigma^{\prime}=\sigma_{3}$ in the following.

Let $\chi:\left(x^{\prime}, y^{\prime}, z^{\prime}, u^{\prime}\right) / \mathbb{Z}_{2} \rightarrow\left(x_{1}, y_{1}, z_{1}, u_{1}\right) / \mathbb{Z}_{2}$ be a liftable automorphism such that $\chi \circ j^{\prime}=j_{1}$ and we dence

$$
p=\chi^{*}\left(x_{1}\right), \quad q=\chi^{*}\left(y_{1}\right), \quad r=\chi^{*}\left(z_{1}\right), \quad s=\chi^{*}\left(u_{1}\right) \in \mathbb{C}\left\{x^{\prime}, y^{\prime}, z^{\prime}, u^{\prime}\right\} .
$$

Then $\varphi^{\prime}=\chi^{*}\left(\varphi_{1}\right)$ is the defining equation of $j^{\prime}$. Since $v^{\prime}>\left(j_{1}, \sigma_{2,1}\right)$, we have

$$
\sigma^{\prime}-w t s\left(\chi^{*}\left(x_{1}, y_{1}, z_{1}, u_{1}\right)\right) \geq \frac{1}{2}(2,3,1,3)
$$


If $y^{\prime} \in s$, then $y^{\prime 2} \in \varphi^{\prime}$ so that $\sigma^{\prime}-w t\left(\varphi^{\prime}\right) \leq 3$, which is a contradiction. Hence $y^{\prime} \not s$. Similarly we see that $z^{\prime 3} \not \subset s$ and $x^{\prime} z^{\prime} \not \subset s$. Thus we get $\sigma^{\prime}-w t(s) \geq 5 / 2$. Let $p=a x^{\prime}+$ $b z^{\prime 2}+$ (other terms) and $q\left(x^{\prime}, y^{\prime}, z^{\prime}, u^{\prime}\right)=c y^{\prime}+d z^{\prime}+$ (other terms). Since $\chi$ is an automorphism and $z^{\prime} \notin r$, we see that $a \neq 0$ and $d \neq 0$. As $\sigma^{\prime}-w t\left(\varphi^{\prime}\right)=5$, we see that the coefficients of $x^{\prime} z^{\prime 4}$ and $z^{\prime 6}$ are both zero. So we have $a b\left(3 b+6 \xi d^{2}\right)=b^{2}(b+$ $\left.3 \xi d^{2}\right)=0$, which shows that $b=0$. Thus we get $\sigma^{\prime}-w t(p) \geq 2$. Therefore $v^{\prime}>v_{3}$.

10.36. By (10.33), (10.34) and (10.35), we see that (4.1), (4.4), (4.5) and (4.6) hold if $h_{\operatorname{deg} 4}(y, z)=y^{3} z$ and if $(\dagger)$ has a solution in $x$. Figure 5(10.D.b) shows the elements of $\mathscr{W}_{1 / 2} / \sim$ and their relations.

10.37. By (10.30) and (10.36), we complete the proof of (4.1), (4.4), (4.5) and (4.6) if $h_{\mathrm{deg}} 4(y, z)$ has a triple and a single factors.

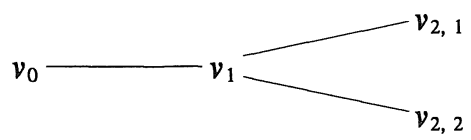

(10.D.a)



(10.D.b)

Figure 5. $\mathscr{W}_{1 / 2}$ for $(\mathrm{cE} / 2)$ type terminal singularities

\section{§ 10.E. $h_{\operatorname{deg} 4}(y, z)$ has a 4-ple factor}

10.38. If $h_{\operatorname{deg}} 4(y, z)$ has a 4-ple factor, we may assume that the standard embedding $j$ is given so that $h_{\operatorname{deg}} 4(y, z)=y^{4}$ by a linear change of $y$ and $z$. As in (10.D), we first consider the system of equations

$$
x^{3}+a_{0,4} x+b_{0,6}=0, \quad 3 x^{2}+a_{0,4}=0, \quad a_{0,6} x+b_{0,8}=0, \quad a_{1,3} x+b_{1,5}=0
$$

in $x$.

10.39. We first assume that $(\dagger)$ has no solutions in $x$ and this case will be treated in (10.40)-(10.43).

10.40. Proposition. For the standard embedding $j$, we have

$$
\mathscr{W}_{1 / 2}(j)=\left\{(j, \sigma) \mid \sigma=\sigma_{0}, \sigma_{1} \text { or } \sigma_{2,1}\right\}
$$

In particular, $v_{2,1}=\left(j, \sigma_{2,1}\right)$ is the unique maximal element in $\mathscr{W}_{1 / 2}(j)$.

Proof. This follows from (10.2) and a similar calculations as in the proof of 
(10.27).

10.41. Theorem. The $v_{2,1}$-blow up $\pi_{1}: \bar{X}_{1} \rightarrow X$ is divisorial with discrepancy $1 / 2$ and $\sum_{Q \in \bar{X}_{1}}\left(\operatorname{aw}\left(\bar{X}_{1}, Q\right)-1\right)=1$. The exceptional divisor of $\pi_{1}$ is the unique divisor with discrepancy $1 / 2$ over $X$.

Proof. By (10.4)(2) and (10.4)(3), we see that $\pi_{1}$ is divisorial with discrepancy $1 / 2$ and that $\sum_{Q \in \bar{X}_{1}}\left(\operatorname{aw}\left(\bar{X}_{1}, Q\right)-1\right)=1$. For the last part, we shall use $\pi_{1}$ as the first blow up. The origin of the $y$-chart of $\bar{X}_{1}$ is of type (cD/3-3) and we can extract the unique divisor $F$ with discrepancy $1 / 3$ over $\bar{X}_{1}$ by the weighted blow up with weight $\frac{1}{3}(2,1,4,3)$. So $a(F, X)=1 / 3+1 / 2 \cdot 4 / 3=1$. Thus $E_{1}$ is the unique divisor with discrepancy $1 / 2$ over $X$.

We can easily prove the following as in the proof of (10.12).

10.42. Proposition. If $v^{\prime}=\left(j^{\prime}, \sigma^{\prime}\right) \in \mathscr{W}_{1 / 2}$ is maximal, then $v^{\prime}>v_{2,1}$.

10.43. By (10.41) and (10.42), we see that (4.1), (4.4), (4.5) and (4.6) hold if $h_{\operatorname{deg} 4}(y, z)=y^{4}$ and if $(\dagger)$ has no solutions in $x$. Figure 6(10.E.a) shows the elements of $\mathscr{W}_{1 / 2} / \sim$ and their relations.

10.44. If $(\dagger)$ has a solution $x=\xi \in \mathbb{C}$, then we shall change the embedding as in (10.31). Let $\chi_{1}:(x, y, z, u) / \mathbb{Z}_{2} \rightarrow\left(x_{1}, y_{1}, z_{1}, u_{1}\right) / \mathbb{Z}_{2}$ be the automorphism defined by

$$
\chi_{1}^{*}\left(x_{1}\right)=x-\xi z^{2}, \quad \chi_{1}^{*}\left(y_{1}\right)=y, \quad \chi_{1}^{*}\left(z_{1}\right)=z \quad \text { and } \quad \chi_{1}^{*}\left(u_{1}\right)=u \text {, }
$$

and let $j_{1}=\chi_{1} \circ j: X \hookrightarrow\left(x_{1}, y_{1}, z_{1}, u_{1}\right) / \mathbb{Z}_{2}(0,1,1,1)$ be the embedding. Then the defining equation of $j_{1}$ is

$$
\varphi_{1}=u_{1}^{2}+x_{1}^{3}+3 \xi x_{1}^{2} z_{1}^{2}+g_{1}\left(y_{1}, z_{1}\right) x_{1}+h_{1}\left(y_{1}, z_{1}\right),
$$

where $\tau$-wt $\left(g_{1}(y, z)\right) \geq 3, \tau-w t\left(h_{1}(y, z)\right) \geq 5$ if we set $\tau$-wt $(y)=3 / 2, \tau$-wt $(z)=$ $1 / 2$. Let

$$
\Phi=u_{1}^{2}+3 \xi x_{1}^{2} y_{1}^{2}+g_{1, \tau-w t=3}\left(y_{1}, z_{1}\right) x_{1}+h_{1, \tau-w t=5}\left(y_{1}, z_{1}\right)
$$

Then the exceptional divisor of the $\left(j_{1}, \sigma_{3}\right)$-blow up of $X$ is isomorphic to $\{\Phi=0\}$ $\subseteq \mathbb{P}(4,1,3,5)$. We divide the cases whether $\Phi$ is irreducible and reduced, or not.

10.45. Under the notation and the assumption (10.44), we further assume that the above $\Phi$ is irreducible and reduced. This case will be treated in (10.46)- 
(10.50).

10.46. Proposition. For the embedding $j_{1}$, we have

$$
\mathscr{W}_{1 / 2}\left(j_{1}\right)=\left\{\left(j_{1}, \sigma\right) \mid \sigma=\sigma_{0}, \sigma_{1}, \sigma_{2,1} \text { or } \sigma_{3}\right\}
$$

In particular, $v_{3}=\left(j_{1}, \sigma_{3}\right)$ is the unique maximal element in $\mathscr{W}_{1 / 2}\left(j_{1}\right)$.

Proof. By (10.2), it is enough to prove that $\left(j_{1}, \sigma_{4}\right),\left(j_{1}, \sigma_{5}\right) \notin \mathscr{W}_{1 / 2}\left(j_{1}\right)$. If $\left(j_{1}, \sigma_{l}\right) \in \mathscr{W}_{1 / 2}\left(j_{1}\right)$ for $l=4$ or 5 , then (3.9.1) implies $\sigma_{l}-w t\left(\varphi_{1}\right) \geq 6$. Thus we get $\Phi=u_{1}^{2}$, which is not our case.

10.47. Theorem. The $v_{3}$-blow up $\pi_{3}: \bar{X}_{3} \rightarrow X$ is divisorial with discrepancy $1 / 2$ and $\sum_{Q \in \bar{X}_{3}}\left(\operatorname{aw}\left(\bar{X}_{3}, Q\right)-1\right)=0$. There are exactly 2 divisors with discrepancies $1 / 2$ over $X$.

Proof. By (10.5)(2) and (10.5)(3), we see that $\pi_{3}$ is divisorial with discrepancy $1 / 2$ and that $\sum_{Q \in \bar{X}_{3}}\left(\operatorname{aw}\left(\bar{X}_{3}, Q\right)-1\right)=0$. For the last part, we can carry out almost the same calculation as in the proof of (10.33). The only difference is the coefficient of $G_{1}$ in $\nu^{*}\left(E_{3}\right)$. In this case, it is $4 / 3$, so we have $a\left(G_{1}, X\right)=1$. Thus $E_{3}$ and $F_{1}$ are the divisors with discrepancies $1 / 2$ over $X$.

10.48. Remark. Among these two divisors with discrepancies $1 / 2$, one is obtained in (10.47), and the other one is obtained as the exceptional divisor of the weighted blow up associated to $v_{2,1} \sim\left(j_{1}, \sigma_{2,1}\right)$. The latter one is not a divisorial blow up as we saw in (10.34).

We can prove the following by a similar argument as in the proof of (10.35).

10.49. Proposition. If $v^{\prime}=\left(j^{\prime}, \sigma^{\prime}\right) \in \mathscr{W}_{1 / 2}$ is maximal, then $v^{\prime}>v_{3}$.

10.50. By (10.47)-(10.49), we see that (4.1), (4.4), (4.5) and (4.6) hold under the assumption (10.45). Figure 6(10.E.b) shows the elements of $\mathscr{W}_{1 / 2} / \sim$ and their relations.

10.51. Next we treat the case $\Phi$ is reducible or not reduced. If $\Phi$ is reducible, then $3 \xi x_{1}^{2} z_{1}^{2}+g_{1, \tau-w t=3}\left(y_{1}, z_{1}\right) x_{1}+h_{1, \tau-w t=5}\left(y_{1}, z_{1}\right)$ is square and we can set

$$
3 \xi x_{1}^{2} z_{1}^{2}+g_{1, \tau-w t=3}\left(y_{1}, z_{1}\right) x_{1}+h_{1, \tau-w t=5}\left(y_{1}, z_{1}\right)=-\left(\alpha x_{1} z_{1}+\beta y_{1} z_{1}^{2}+\gamma z_{1}^{5}\right)^{2}
$$

for some $\alpha, \beta, \gamma \in \mathbb{C}$. Let $\chi_{ \pm}:\left(x_{1}, y_{1}, z_{1}, u_{1}\right) / \mathbb{Z}_{2} \rightarrow\left(x_{2}, y_{2}, z_{2}, u_{2}\right) / \mathbb{Z}_{2}$ be the automorphisms defined by 


$$
\begin{aligned}
& \chi_{ \pm}^{*}\left(x_{2}\right)=x_{1}, \quad \chi_{ \pm}^{*}\left(y_{2}\right)=y_{1}, \quad \chi_{ \pm}^{*}\left(z_{2}\right)=z_{1} \\
& \text { and } \quad \chi_{ \pm}^{*}\left(u_{2}\right)=u_{1} \pm\left(\alpha x_{1} z_{1}+\beta y_{1} z_{1}^{2}+\gamma z_{1}^{5}\right),
\end{aligned}
$$

and let $j_{ \pm}=\chi={ }^{\circ} j_{1}: X \hookrightarrow\left(x_{2}, y_{2}, z_{2}, u_{2}\right) / \mathbb{Z}_{2}(0,1,1,1)$ be the embeddings. Then their defining equations are

$$
\varphi_{ \pm}=u_{2}^{2} \mp 2\left(\alpha x_{2} z_{2}+\beta y_{2} z_{2}^{2}+\gamma z_{2}^{5}\right) u_{2}+x_{2}^{3}+g_{2}\left(y_{2}, z_{2}\right) x_{2}+h_{2}\left(y_{2}, z_{2}\right)
$$

where $g_{2}(y, z)=g_{1, \tau-w t \geq 4}(y, z), h_{2}(y, z)=h_{1, \tau-w t \geq 6}(y, z)$. We denote the power series expansion of $g_{2}(y, z)$ and $h_{2}(y, z)$ by $g_{2}(y, z)=\sum_{i, j} a_{i, j}^{(2)} y^{i} z^{j}$ and $h_{2}(y, z)=$ $\sum_{i, j} b_{i, j}^{(2)} y^{i} z^{j}$ respectively.

10.52. Under the notation and the assumption (10.51), we further assume


treat this case in (10.53)-(10.57).

10.53. Proposition. For each embedding $j_{ \pm}$, we have

$$
\mathscr{W}_{1 / 2}\left(j_{ \pm}\right)=\left\{\left(j_{ \pm}, \sigma\right) \mid \sigma=\sigma_{0}, \sigma_{1}, \sigma_{2,1}, \sigma_{3} \text { or } \sigma_{4}\right\}
$$

In particular, $v_{ \pm}=\left(j_{ \pm}, \sigma_{4}\right)$ is the unique maximal element in $\mathscr{W}_{1 / 2}\left(j_{ \pm}\right)$.

Proof. If $\left(j_{ \pm}, \sigma_{5}\right) \in \mathscr{W}_{1 / 2}\left(j_{ \pm}\right)$, then (3.9.1) implies $\sigma_{5}-w t\left(\varphi_{ \pm}\right)=9$. On the other hand, we easily see that $\sigma_{5}-w t\left(\varphi^{\prime}\right) \leq \sigma_{5}-w t\left(\left(\alpha x_{2} y_{2}+\beta y_{2}^{5}+\gamma y_{2}^{2} z_{2}\right) u_{2}\right) \leq 8$, which is a contradiction.

10.54. Theorem. Let $\pi_{ \pm}: \bar{X}_{ \pm} \rightarrow X$ be the $v_{ \pm}$-blow ups. Then $\pi_{ \pm}$are both divisorial with discrepancies $1 / 2$ and $\sum_{Q \in \bar{X}_{ \pm}}\left(\operatorname{aw}\left(\bar{X}_{ \pm}, Q\right)-1\right)=0$. These $\pi_{+}$and $\pi_{-}$ are not isomorphic over $X$. Furthermore, there are exactly 3 divisors with discrepancies $1 / 2$ over $X$.

Proof. By (10.6)(1) and (10.6)(3), we see that $\pi_{ \pm}$are both divisorial with discrepancies $1 / 2$ and $\Sigma_{Q \in \bar{X}_{ \pm}}\left(\operatorname{aw}\left(\bar{X}_{ \pm}, Q\right)-1\right)=0$.

For the last part, we shall use $\pi_{+}$as the first blow up. Let $E_{+}$be the exceptional divisor of $\pi_{+}$. The origin $Q$ of the $u_{2}$-chart of $\bar{X}_{+}$is the unique non Gorenstein point. We can resolve this by (5.1) and get a projective birational morphism $\nu: Z \rightarrow \bar{X}_{+}$such that $K_{Z}=\nu^{*}\left(K_{\bar{X}_{+}}\right)+\sum_{i=1}^{6} \frac{i}{7} F_{i}$, where $\sum F_{i}$ is the exceptional divisor of $\nu$ over $Q$. By (10.5) (3) and (5.1),

$$
\nu^{*}\left(E_{+}\right)=\nu^{-1}\left[E_{+}\right]+\frac{5}{7} F_{1}+\frac{3}{7} F_{2}+\frac{8}{7} F_{3}+\frac{6}{7} F_{4}+\frac{4}{7} F_{5}+\frac{9}{7} F_{6} .
$$


Therefore $a\left(F_{1}, X\right)=a\left(F_{2}, X\right)=1 / 2$ and $a\left(F_{i}, X\right) \geq 1$ for $i \geq 3$. Thus $E_{+}, F_{1}$ and $F_{2}$ are the divisors with discrepancies $1 / 2$ over $X$.

10.55. Remark. Among these three divisors with discrepancies 1/2, two of them are obtained in (10.54), and the remaining one is obtained as the exceptional divisor of the weighted blow up associated to $v_{2,1} \sim\left(j_{ \pm}, \sigma_{2,1}\right)$. But this is not a divisorial blow up as we saw in (10.35).

10.56. Proposition. If $v^{\prime}=\left(j^{\prime}, \sigma^{\prime}\right) \in \mathscr{W}_{1 / 2}$ is maximal, then $v^{\prime}>v+$ or $v^{\prime}>$ $v_{-}$.

Proof. We shall show that $v^{\prime}>v_{-}$assuming $v^{\prime} \Varangle_{v_{+}}$. Let $\chi:\left(x^{\prime}, y^{\prime}, z^{\prime}, u^{\prime}\right) /$ $\mathbb{Z}_{2} \rightarrow\left(x_{2}, y_{2}, z_{2}, u_{2}\right) / \mathbb{Z}_{2}$ be a liftable automorphism such that $\chi \circ j^{\prime}=j_{+}$. Then $\varphi^{\prime}$ $=\chi^{*}\left(\varphi_{+}\right)$is the defining equation of $j^{\prime}$. By (10.49), we see that $v^{\prime}>v_{3}=\left(j_{1}, \sigma_{3}\right) \sim$ $\left(j_{+}, \sigma_{3}\right)$. We may assume that $\sigma^{\prime}=\sigma_{4}$ since otherwise $v^{\prime}$ is not maximal by $(5.5)$. Thus (3.9.1) implies that $\sigma^{\prime}-w t\left(\varphi^{\prime}\right)=6$. Since $v^{\prime}>\left(j_{+}, \sigma_{3}\right)$, we also have

$$
\sigma^{\prime}-w t s\left(\chi^{*}\left(x_{2}, y_{2}, z_{2}, u_{2}\right)\right) \geq \frac{1}{2}(4,3,1,5) \text {. }
$$

By our assumption, we have $\sigma^{\prime}-w t\left(\chi^{*}\left(u_{2}\right)\right)=5 / 2$. Thus $\sigma^{\prime}-w t\left(\varphi^{\prime}\right)=6$ implies that $\sigma^{\prime}-w t\left(\chi^{*}\left(u_{2}-2\left(\alpha x_{2} z_{2}+\beta y_{2} z_{2}^{2}+\gamma z_{2}^{5}\right)\right)\right) \geq 7 / 2$, which shows that $v^{\prime}>v_{-}$.

10.57. By (10.54)-(10.56), we see that (4.1), (4.4), (4.5) and (4.6) hold under the assumption (10.52). Figure 6(10.E. c) shows the elements of $\mathscr{W}_{1 / 2} / \sim$ and their relations.

10.58. Under the notation and the assumption (10.51), we next assume $\alpha x_{1} z_{1}$ $+\beta y_{1} z_{1}^{2}+\gamma z_{1}^{5}=0$. We see that $j_{+}=j_{-}$in this case. So we shall denote the common embedding by $j_{2}$ and its defining equation by $\varphi_{2}$. We also denote $v_{4}=\left(j_{2}, \sigma_{4}\right)$. Let $\pi_{4}: \bar{X}_{4} \rightarrow X$ be the $v_{4}$-blow up and $E_{+}$be the exceptional divisor of $\pi_{4}$. By (10.6) (2), $\bar{X}_{4}$ has only canonical singularities and $E_{+}$is irreducible. We also see that $\bar{X}_{4}$ is terminal outside the $z_{2}$-chart $U_{3}$ of $\bar{X}_{4}$ :

$$
U_{3}=\left\{\bar{u}^{2} \bar{z}+\bar{x}^{3}+g_{2}\left(\bar{y} \bar{z}^{3 / 2}, \bar{z}^{1 / 2}\right) / \bar{z}^{4} \cdot \bar{x}+h_{2}\left(\bar{y} \bar{z}^{3 / 2}, \bar{z}^{1 / 2}\right) / \bar{z}^{6}=0\right\} \subseteq \mathbb{C}^{4}
$$

We divide the case whether $\bar{X}_{4}$ has only terminal singularities or not.

10.59. Under the notation and the assumption (10.58), we first assume that $\bar{X}_{4}$ has only terminal singularities. This case will be studied in (10.60)-(10.64).

10.60. Proposition. For the embedding $j_{2}$, we have 


$$
\mathscr{W}_{1 / 2}\left(j_{2}\right)=\left\{\left(j_{2}, \sigma\right) \mid \sigma=\sigma_{0}, \sigma_{1}, \sigma_{2,1}, \sigma_{3} \text { or } \sigma_{4}\right\}
$$

In particular, $v_{4}=\left(j_{2}, \sigma_{4}\right)$ is the unique maximal element in $\mathscr{W}_{1 / 2}\left(j_{2}\right)$.

Proof. Assume that $\left(j_{2}, \sigma_{5}\right) \in \mathscr{W}_{1 / 2}\left(j_{2}\right)$. We see from (3.9.1) that $\sigma_{5}-w t\left(\varphi_{2}\right)$ $=9$, hence $a_{i, j}^{(2)}=0$ if $5 i+j \leq 12$ and $b_{i, j}^{(2)}=0$ if $5 i+j \leq 16$. These conditions imply that the origin of the $z_{2}$-chart $U_{3}$ of $\bar{X}_{4}$ is non-terminal.

10.61. Theorem. The $v_{4}$-blow up $\pi_{4}: \bar{X}_{4} \rightarrow X$ is divisorial with discrepancy $1 / 2$ and $\sum_{Q \in \bar{X}_{4}}\left(\operatorname{aw}\left(\bar{X}_{4}, Q\right)-1\right)=0$. There are exactly 2 divisors with discrepancies $1 / 2$ over $X$.

Proof. Since $E_{+}$is irreducible, the first part follows. For the second part, we can do almost the same calculation as in the proof of (10.54). The only difference is the coefficient of $F_{1}$ in $\nu^{*}\left(E_{+}\right)$. In this case, it is $12 / 7$ and $a\left(F_{1}, X\right)=1$. Hence $E_{+}$and $F_{2}$ are the divisors with discrepancies $1 / 2$ over X.

10.62. Remark. Among these two divisors with discrepancies $1 / 2$. One is obtained in (10.61), and the other one obtained as the exceptional divisor of the weighted blow up associated to $v_{2,1} \sim\left(j_{2}, \sigma_{2,1}\right)$. The latter one is not a divisorial blow up as we saw in (10.34).

We can prove the following by almost the same method as (10.56).

10.63. Proposition. If $v^{\prime}=\left(j^{\prime}, \sigma^{\prime}\right) \in \mathscr{W}_{1 / 2}$ is maximal, then $v^{\prime}>v_{4}$.

10.64. By (10.61)-(10.63), we see that (4.1), (4.4), (4.5) and (4.6) hold under the assumption (10.59). Figure 6(10.E.d) shows the elements of $\mathscr{W}_{1 / 2} / \sim$ and their relations.

10.65. Under the notation and the assumption (10.58), we next assume that $\bar{X}_{4}$ has non-terminal singularities. We shall study this case in (10.66)-(10.70). In this case, we first analyse the non-terminal singularities more closely. By (10.6) (2), we need to study only the $z_{2}$-chart $U_{3}$ of $\bar{X}_{4}$. We see from the description of $U_{3}$ that the non-terminal singularities in $U_{3}$ lies in $\{\bar{y}=\delta, \bar{z}=0\}$ for some $\delta \in \mathbb{C}$. Let $\chi_{3}:\left(x_{2}, y_{2}, z_{2}, u_{2}\right) / \mathbb{Z}_{2} \rightarrow\left(x_{3}, y_{3}, z_{3}, u_{3}\right) / \mathbb{Z}_{2}$ be the automorphism defined by

$$
\chi_{3}^{*}\left(x_{3}\right)=x_{2}, \quad \chi_{3}^{*}\left(y_{3}\right)=y_{2}-\delta z_{2}^{3}, \quad \chi_{3}^{*}\left(z_{3}\right)=z_{2} \quad \text { and } \quad \chi_{3}^{*}\left(u_{3}\right)=u_{2},
$$

and let $j_{3}=\chi_{3} \circ j_{2}: X \hookrightarrow\left(x_{3}, y_{3}, z_{3}, u_{3}\right) / \mathbb{Z}_{2}(0,1,1,1)$ be the embedding. Then the defining equation of $j_{3}$ is 


$$
\varphi_{3}=u_{3}^{2}+x_{3}^{3}+g_{3}\left(y_{3}, z_{3}\right) x_{3}+h_{3}\left(y_{3}, z_{3}\right) \text {, }
$$

where $g_{3}(y, z)=g_{2}\left(y+\delta z^{3}, z\right), h_{3}(y, z)=h_{2}\left(y+\delta z^{3}, z\right)$. We denote the power series expansion of $g_{3}(y, z)$ and $h_{3}(y, z)$ by $g_{3}(y, z)=\sum_{i, j} a_{i, j}^{(3)} y^{i} z^{j}$ and $h_{3}(y, z)=$ $\sum_{i, j} b_{i, j}^{(3)} y^{i} z^{j}$ respectively.

Now let $\pi_{4}^{\prime}: \bar{X}_{4}^{\prime} \rightarrow X$ be the $v_{4}^{\prime}$-blow up. We have $\bar{X}_{4}^{\prime} \simeq \bar{X}_{4}$ since $v_{4} \sim v_{4}^{\prime}$. Thus the $z_{3}$-chart of $\bar{X}_{4}^{\prime}$ has non-terminal singularities. Moreover, these lies in $\bar{y}=\bar{z}=0$. Thus, in addition to the conditions $\tau$-wt $\left(g_{3}(y, z)\right) \geq 4$ and $\tau$-wt $\left(h_{3}(y, z)\right) \geq 6$, we see that

$$
a_{1,5}^{(3)}=a_{0,8}^{(3)}=a_{0,10}^{(3)}=b_{2,6}^{(3)}=b_{1,9}^{(3)}=b_{1,11}^{(3)}=b_{0,12}^{(3)}=b_{0,14}^{(3)}=0,
$$

which implies that $\tau^{\prime}-w t\left(g_{3}(y, z)\right) \geq 6$ and $\tau^{\prime}-w t\left(h_{3}(y, z)\right) \geq 9$ if we set $\tau^{\prime}-w t(y)=$ $5 / 2, \tau^{\prime}-w t(z)=1 / 2$.

10.66. Proposition. For the embedding $j_{3}$, we have

$$
\mathscr{W}_{1 / 2}\left(j_{3}\right)=\left\{\left(j_{3}, \sigma\right) \mid \sigma=\sigma_{0}, \sigma_{1}, \sigma_{2,1}, \sigma_{3}, \sigma_{4} \text { or } \sigma_{5}\right\}
$$

In particular, $v_{5}=\left(j_{3}, \sigma_{5}\right)$ is the unique maximal element in $\mathscr{W}_{1 / 2}\left(j_{3}\right)$.

10.67. Theorem. The $v_{5}$-blow up $\pi_{5}: \bar{X}_{5} \rightarrow X$ is divisorial with discrepancy $1 / 2$ and $\sum_{Q \in \bar{X}_{5}}\left(\operatorname{aw}\left(\bar{X}_{5}, Q\right)-1\right)=0$. There are exactly 3 divisors discrepancies $1 / 2$ over $X$.

Proof. We see that $\pi_{5}: \bar{X}_{5} \rightarrow X$ is divisorial with discrepancy $1 / 2$ and that $\Sigma_{Q \in \bar{X}_{5}}\left(\operatorname{aw}\left(\bar{X}_{5}, Q\right)-1\right)=0$ by (10.7). Let $E_{5}$ be the exceptional divisor of $\pi_{5}$. By (5.1), there is a projective birational morphism $\nu: Z \rightarrow \bar{X}_{5}$ such that, which is a resolution of the origin of the $\bar{y}$-chart of $\bar{X}_{5}$, such that $K_{Z}=\nu^{*}\left(K_{\bar{X}_{5}}\right)+\sum_{i=1}^{4} \frac{i}{5} F_{i}$, where $\sum_{i=1}^{4} F_{i}$ is the exceptional divisor of $\nu$. By (10.7) and (5.1), we have

$$
\nu^{*}\left(E_{5}\right)=\nu^{-1}\left[E_{5}\right]+\frac{3}{5} F_{1}+\frac{6}{5} F_{2}+\frac{4}{5} F_{3}+\frac{2}{5} F_{4} .
$$

Hence $a\left(F_{1}, X\right)=1 / 2$ and $a\left(F_{i}, X\right) \geq 1$ for $i \geq 2$. There is also an index 3 point on $\bar{X}_{5}$, so there are at most 3 divisors with discrepancies $1 / 2$ over $X$. On the other hand, as we shall soon see in (10.68), there are at least 3 divisors with discrepancies $1 / 2$ over $X$.

10.68. Remark. Among these three divisors with discrepancies $1 / 2$, one is obtained in (10.67), others are obtained as the exceptional divisors of the $\left(j, \sigma_{2,1}\right)$ blow up and the $\left(j_{3}, \sigma_{4}\right)$-blow up. 
10.69. Proposition. If $v^{\prime}=\left(j^{\prime}, \sigma^{\prime}\right) \in \mathscr{W}_{1 / 2}$ is maximal, then $v^{\prime}>v_{5}$.

Proof. Let $\chi:\left(x^{\prime}, y^{\prime}, z^{\prime}, u^{\prime}\right) / \mathbb{Z}_{2} \rightarrow\left(x_{3}, y_{3}, z_{3}, u_{3}\right) / \mathbb{Z}_{2}$ be a liftable automorphism such that $\chi \circ j_{3}=j^{\prime}$. By (10.63), we see that $v^{\prime}>v_{4}=\left(j_{2}, \sigma_{4}\right) \sim\left(j_{3}, \sigma_{4}\right)$. Thus we have

$$
\sigma^{\prime}-w t s\left(\chi^{*}\left(x_{3}, y_{3}, z_{3}, u_{3}\right)\right) \geq \frac{1}{2}(4,3,1,7) \text {. }
$$

For the defining equation $\varphi^{\prime}=\chi^{*}\left(\varphi_{3}\right)$, we have $\sigma^{\prime}-w t\left(\varphi^{\prime}\right)=9$ by (3.9.1). Let

$$
p=\chi^{*}\left(x_{3}\right), \quad q=\chi^{*}\left(y_{3}\right), \quad r=\chi^{*}\left(z_{3}\right), \quad s=\chi^{*}\left(u_{3}\right) \in \mathbb{C}\left\{x^{\prime}, y^{\prime}, z^{\prime}, u^{\prime}\right\} .
$$

Since $y^{\prime} \notin s$ and $z^{\prime} \notin s$, we have $u^{\prime} \in s$.

If $x^{\prime} z^{\prime} \in s$, then $x^{\prime} z^{\prime} u^{\prime} \in \varphi^{\prime}$, so we get $\sigma^{\prime}-w t\left(\varphi^{\prime}\right) \leq 8$, which is a contradiction. Thus we know that $x^{\prime} z^{\prime} \notin \varphi^{\prime}$. Similarly, we see that $y^{\prime} z^{\prime 2} \notin s$. Assume that $z^{\prime 4} \in p$. Since $x^{\prime} \in p$, we see that $x^{\prime 2} z^{\prime 4} \in \varphi^{\prime}$, so that $\sigma^{\prime}-w t\left(\varphi^{\prime}\right) \leq 8$, which is a contradiction. Hence $z^{\prime 4} \notin p$, which shows that $\sigma^{\prime}-w t(p) \geq 3$. By a similar argument, we see that $z^{\prime 3} \not \subset r$ and $z^{\prime 7} \not s$, which shows that $\sigma-w t(r) \geq 5 / 2$ and $\sigma^{\prime}-w t(s) \geq 9 / 2$.

10.70. By (10.67)-(10.69), we see that (4.1), (4.4), (4.5) and (4.6) hold under the assumption (10.65). Figure 6(10.E.e) shows the elements of $\mathscr{W}_{1 / 2} / \sim$ and their relations.

10.71. Thus we complete the proof of (4.1), (4.4), (4.5) and (4.6) if $h_{\operatorname{deg} 4}(y, z)$ has a 4-ple factor.
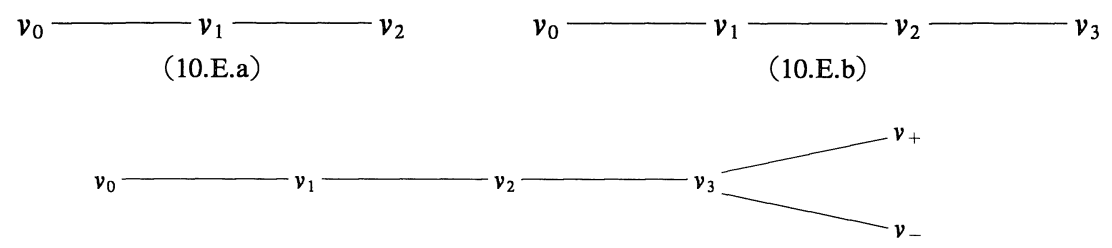

(10.E.c)

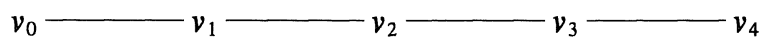

(10.E.d)



Figure 6. $\mathscr{W}_{1 / 2}$ for $(\mathrm{cE} / 2)$ type terminal singularities 


\section{References}

[Cor95] Corti, A., Factoring birational maps of threefolds after Sarkisov, J. Algebraic Geom., 4 (1995), 223-254.

[Cut88] Cutkosky, S., Elementary contractions of Gorenstein threefolds, Math. Ann., 280 (1988), 521-525.

[Dan83] Danilov, V., Birational geometry of toric 3-folds, Math. USSR Izv., 21 (1983), 269-280.

[Ful93] Fulton, W., Introduction to toric varieties, Princeton Univ. Press, 1993.

[Kaw86] Kawamata, Y., On the plurigenera of minimal algebraic 3-folds with $K \approx 0$, Math. Ann., 275 (1986), 539-546.

[Kaw93] The minimal discrepancy of a 3-fold terminal singularity, Appendix to [Sho 93]

[Kaw96] - Divisorial contractions to 3-dimensional terminal quotient singularities, Higher-dimensional complex varieties (Trento, 1994), de Gruyter, 1996, 241-246.

[KMM87] Kawamata, Y., Matsuda, K. and Matsuki, K., Introduction to the minimal model problem, Algebraic Geometry, Sendai, 1985, Adv. Stud. Pure Math., North-Holland, 10 (1987), 283-360.

[Ko197] Kollár, J., Real algebraic threefolds II. Minimal model program, preprint.

[FA92] Kollár, J. et al., Flips and abundance for algebraic threefolds, Astérisque, 211 (1992).

[KM92] Kollár, J. and Mori, S., Classification of three dimensional flips, J. Amer. Math. Soc., 5 (1992), 533-703.

[KSB88] Kollár, J. and Shepard-Barron, N., Threefolds and deformation of surface singularities, Inv. Math., 91 (1988), 299-338.

[Luo98] Luo, T., Divisorial extremal contractions of threefolds : divisor to point, Amer. J. Math., 120 (1998), 441-451.

[Mar96] Markushevich, D., Minimal discrepancies for a terminal cDV singularities is 1, J. Math. Sci. Univ. Tokyo, 3 (1996), 445-456.

[Mori82] Mori, S., Threefolds whose canonical bundles are not numerically effective, Ann. of Math., 116 (1982), 133-176.

[Mori85] - On 3-dimensional terminal singularities, Nagoya Math. J., 98 (1985), 43-66.

[Mori88] - Flip theorem and the existence of minimal models for 3-folds, J. Amer. Math. Soc., 1 (1988), 117-253.

[Morr86] Morrison, D., A remark on Kawamata's paper "On the plurigenera of minimal algebraic 3-folds with $K \approx 0$ ”, Math. Ann., 275 (1986), 547-553.

[MS84] Morrison, D. and Stevens, G., Terminal quotient singularities in dimension three and four, Proc. Amer. Math. Soc., 90 (1984), 15-20.

[Oda88] Oda, T., Convex bodies and algebraic geometry, Springer-Verlag, 1988.

[Reid80] Reid, M., Canonical threefolds, Géométrie Algébrique Angers (A. Beauville, ed.), Sijthoff \& Noordhoff, 1980, 273-310.

[Reid83] - Minimal models of canonical threefolds, Algebraic Varieties and Analytic Varietiese, Adv. Stud. Pure Math., Kinokuniya and North-Holland, 1 (1983), 131-180.

[Reid87] - Young person's guide to canonical singularities, Algebraic Geometry, Bowdoin 1985, Proc. Symp. Pure Math., 46 (1987), 345-416.

[Sho93] Shokurov, V., 3-fold log flips, Russian Acad. Sci. Izu. Math., 40 (1993), 95-202.

[Sho96] - 3-fold log models, J. Math. Sci., 81 (1996), 2667-2699.

[Ste88] Stevens, J., On canonical singularities as total spaces of deformations, Abh. Math. Sem. Univ. Hamburg, 58 (1988), 275-283. 